\title{
Incorporation of sensing modalities into de novo designed fluorescence-activating proteins
}

Jason C. Klima (1) 1,2, Lindsey A. Doyle (1) 3,13, Justin Daho Lee (i) 4,5,6,13, Michael Rappleye (i) 5,6, Lauren A. Gagnon7, Min Yen Lee (10 7, Emilia P. Barros ${ }^{8}$, Anastassia A. Vorobieva (1) 1,2, Jiayi Dou 1,2,12, Samantha Bremner (10 5,6, Jacob S. Quon (10 2, Cameron M. Chow (1) 1,2, Lauren Carter 1,2, David L. Mack 5,6,9, Rommie E. Amaro (1) 8, Joshua C. Vaughan (10) 7,10, Andre Berndt 4,5,6, Barry L. Stoddard ${ }^{3}$ \& David Baker (1) 1,2,4,11凶

Through the efforts of many groups, a wide range of fluorescent protein reporters and sensors based on green fluorescent protein and its relatives have been engineered in recent years. Here we explore the incorporation of sensing modalities into de novo designed fluorescence-activating proteins, called mini-fluorescence-activating proteins (mFAPs), that bind and stabilize the fluorescent cis-planar state of the fluorogenic compound DFHBI. We show through further design that the fluorescence intensity and specificity of mFAPs for different chromophores can be tuned, and the fluorescence made sensitive to $\mathrm{pH}$ and $\mathrm{Ca}^{2+}$ for real-time fluorescence reporting. Bipartite split mFAPs enable real-time monitoring of protein-protein association and (unlike widely used split GFP reporter systems) are fully reversible, allowing direct readout of association and dissociation events. The relative ease with which sensing modalities can be incorporated and advantages in smaller size and photostability make de novo designed fluorescence-activating proteins attractive candidates for optical sensor engineering.

\footnotetext{
${ }^{1}$ Department of Biochemistry, University of Washington, Seattle, WA, USA. ${ }^{2}$ Institute for Protein Design, University of Washington, Seattle, WA, USA. ${ }^{3}$ Division of Basic Sciences, Fred Hutchinson Cancer Research Center, Seattle, WA, USA. ${ }^{4}$ Molecular Engineering Ph.D. Program, University of Washington, Seattle, WA, USA. ${ }^{5}$ Institute for Stem Cell and Regenerative Medicine, University of Washington, Seattle, WA, USA. ${ }^{6}$ Department of Bioengineering, University of Washington, Seattle, WA, USA. ${ }^{7}$ Department of Chemistry, University of Washington, Seattle, WA, USA. ${ }^{8}$ Department of Chemistry and Biochemistry, University of California, San Diego, La Jolla, CA, USA. ${ }^{9}$ Department of Rehabilitation Medicine, University of Washington, Seattle, WA, USA. ${ }^{10}$ Department of Physiology and Biophysics, University of Washington, Seattle, WA, USA. ${ }^{11}$ Howard Hughes Medical Institute, University of Washington, Seattle, WA, USA. ${ }^{12}$ Present address: Department of Bioengineering, Stanford University, Stanford, CA, USA. ${ }^{13}$ These authors contributed equally: Lindsey A. Doyle, Justin Daho Lee. ${ }^{凶}$ email: dabaker@uw.edu
} 


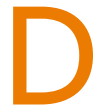
e novo designed mini-fluorescence-activating proteins (mFAPs) (Fig. 1a) bind and activate the fluorescence of the fluorogenic compound DFHBI (3,5-difluoro-4hydroxybenzylidene imidazolinone) (1, Fig. 1b) in vitro and in bacteria, yeast, and mammalian cells ${ }^{1}$. DFHBI does not fluoresce when free in solution ${ }^{2}$, but becomes brightly fluorescent upon stabilization of the cis-planar conformation (planar $\mathrm{Z}$ conformation) through macromolecular binding ${ }^{3}$. RNA aptamers have been evolved to bind similar fluorogenic DFHBI-derived compounds $^{4,5}$ (e.g., DFHBI, DFHBI-1T [(Z)-4-(3,5-difluoro4-hydroxybenzylidene)-2-methyl-1-(2,2,2-trifluoroethyl)-1H-imidazol-5(4 H)-one] (2, Fig. 1c) and DFHO [3,5-difluoro-4hydroxybenzylidene imidazolinone-2-oxime]), with up to 0.72 fluorescence quantum yield ${ }^{6}$, but to our knowledge so far no protein-based systems other than the mFAPs have been reported to bind and fluorescently activate DFHBI-1T or DFHO chromophores.

Circularly permuted fluorescent proteins such as cpGFP and cpFAST enable the real-time detection of analytes of interest using fluorescence microscopy ${ }^{7-9}$. Likewise, self-complementing split fluorescent protein reporter systems based on green fluorescent protein (GFP) variants ${ }^{10-12}$ and FAST $^{13}$ have been engineered to monitor protein-protein interactions in vitro, in cyto, and in vivo ${ }^{14}$ using fluorescence microscopy. The $\beta$-barrel structure of mFAPs suggests that mFAPs could be re-designed to monitor analyte fluxes and protein-protein interactions; such sensors could have complementary biophysical properties to existing fluorescent proteins (such as intrinsically fluorescent GFP and extrinsically fluorogenic $\mathrm{DiBs}^{15}$, Y-FAST ${ }^{16}, \mathrm{Ca}^{2+}$-responsive $\mathrm{cpFAST}^{7}$, and splitFAST ${ }^{13}$ reporters and sensors).

mFAPs have several biophysical properties that make them attractive candidates for further development. First, they are less than half the size of GFP, so their genetic footprint is smaller, and fusions to proteins of interest are less perturbative. Second, the bound chromophore can readily exchange with free chromophore in solution, and hence mFAPs can be more photostable ${ }^{17}$ than GFP. Third, chemical derivatives of DFHBI with different fluorescence properties can be fluorescently activated, providing more control over color (i.e., fluorescence excitation and emission wavelength) than intrinsically fluorescent proteins ${ }^{7}$. Fourth, de novo mFAPs can be engineered to remain folded at low $\mathrm{pH}$, facilitating the engineering of $\mathrm{pH}$-responsive fluorogenic optical sensors. Finally, the chromophore-binding pocket is close to the protein surface, potentially enabling the design of allosteric coupling between chromophore binding and linked analyte binding domains for analyte-responsive fluorogenic optical sensors ${ }^{7,18,19}$.

Here, we explore the incorporation of sensing modalities into the mFAPs. We develop and apply methodologies for engineering chromophore-selective, $\mathrm{pH}$-responsive, $\mathrm{Ca}^{2+}$-responsive, bipartite, and circularly permuted optical sensors based on de novo designed fluorescence-activating proteins.

\section{Results}

Optimizing brightness and chromophore selectivity. We began by seeking to improve the stability of mFAPs at low $\mathrm{pH}$, the binding affinity to the phenolic and phenolate forms of DFHBI, as well as the fluorescence intensity of both complexes. mFAP2 was chosen for optimization because it has the highest absolute fluorescence quantum yield $\left(\phi_{\mathrm{c}}\right.$ of $\left.2.1 \%\right)$ and highest affinity $\left(K_{\mathrm{d}}\right.$ of $\sim 180 \mathrm{nM}$ ) for the phenolate form of DFHBI compared to mFAP 1 . Through a series of library selections (see "Methods") targeting aliphatic and aromatic residues directly interacting with DFHBI or in the hydrophobic core of the $\beta$-barrel, as well as residues in the loop connecting the seventh and eighth $\beta$-strands (loop7) of the $\beta$-barrel, we obtained three brighter and chromophore-selective mFAP2 variants: mFAP2a, mFAP2b, and mFAP10 that incorporate 12,10 , and 11 mutations, respectively (Supplementary Fig. 1).

Titrations of mFAP2, mFAP2a, mFAP2 $b$, and mFAP10 with either DFHBI (Fig. 1d) or DFHBI-1T (Fig. 1e) and quantum yield measurements (Table 1 and Supplementary Fig. 2) showed that: mFAP2, mFAP2a, and mFAP10 have 2 .7-fold, $\sim 2.5$-fold, and $\sim 12$-fold brighter fluorescence with DFHBI-1T than DFHBI, but bind DFHBI with $\sim 30$-fold, $\sim 39$-fold, and $\sim 2.6$-fold higher affinity than DFHBI-1T, respectively; and that mFAP2b has $\sim 30$ fold brighter fluorescence with DFHBI than DFHBI-1T and binds DFHBI with $\sim 6.1$-fold higher affinity than DFHBI-1T. The mFAP10-DFHBI-1T complex is the brightest, with $23.7 \%$ absolute quantum yield (under conditions with $99.9 \%$ of chromophore bound) and a 17.5-fold increased brightness over the previously reported $\mathrm{mFAP} 2-\mathrm{DFHBI}$ complex ${ }^{1}$, resulting in a 242 -fold fluorescence activation over free DFHBI-1T (Table 1). Relative fluorescence intensities and thermodynamic dissociation constants $\left(K_{\mathrm{d}}\right)$ for the deprotonated (phenolate) states of DFHBI, DFHBI-1T, and DFHO for the $\mathrm{Ca}^{2+}$-independent mFAP variants presented in this study are given in Supplementary Table 1 (for example, mFAP3 binds the yellow colored DFHO chromophore with $\sim 10$-fold lower fluorescence intensity than mFAP2b with DFHBI). Using a laser scanning confocal fluorescence microscope to image $E$. coli expressing either mFAP2a or mFAP2b labeled with either DFHBI or DFHBI-1T, we observed pronounced chromophore selectivity of mFAP2b for DFHBI over DFHBI-1T, and chromophore promiscuity of mFAP2a for both DFHBI or DFHBI-1T (Fig. 1f-i). E. coli cultures expressing either mFAP2a or mFAP2b mixed in a 1:1 cellular ratio labeled with DFHBI-1T have $\sim 49 \%$ of the total fluorescence signal of cultures labeled with DFHBI (Supplementary Fig. 3).

We next targeted mFAP2a or mFAP2b to the endoplasmic reticulum (ER) of mammalian COS-7 cells using a C-terminal sec61 $\beta$ localization sequence, and observed bright fluorescence of ER under fixed cell (Supplementary Fig. 4) and live cell (Supplementary Movie 1 and Supplementary Movie 2) epifluorescence microscopy after labeling with DFHBI. Under fixed cell imaging, following washing and re-labeling with DFHBI-1T (Supplementary Fig. 4) the fluorescence was altered as expected, demonstrating external spatiotemporal control over fluorescence. To compare the photostability of mFAP2a and mFAP2b to a monomeric enhanced GFP (EGFP) variant, we also targeted AcGFP1 to the ER of COS-7 cells. Upon continuous wave illumination imaging at $\sim 0.885 \mathrm{~Hz}\left(1.13 \mathrm{~s}_{\text {frame }}{ }^{-1}\right)$ of fixed COS7 cells using laser scanning confocal fluorescence microscopy, we found at $50.0 \mu \mathrm{M}$ chromophore (saturating conditions) a 6.2 -fold, 3.5-fold, and 6.1-fold improved photostability of mFAP2a-DFHBI, mFAP2a-DFHBI-1T, and mFAP2b-DFHBI complexes over AcGFP1, respectively. At $500 \mathrm{nM}$ chromophore (sub-saturating conditions), the improvements in photostability of the three complexes over AcGFP1 were 5.0-fold, 3.8-fold, and 4.7-fold, respectively (Fig. 2).

Incorporation of $\mathbf{p H}$-responsiveness. A prerequisite for designing a robust $\mathrm{pH}$-responsive $\mathrm{mFAP}$ is the ability to bind both protonated (phenolic) DFHBI tautomers and both deprotonated (phenolate) DFHBI resonance structures (Fig. 3a). When stabilized in the cis-planar conformation, the phenolic and phenolate forms of DFHBI exhibit blueshifted and redshifted peak excitation wavelengths, respectively ${ }^{6}$ (Fig. $3 \mathrm{~d}$ ). mFAP2b binds both forms of DFHBI (Fig. 3g); to increase pH sensitivity we screened design variants based on the change in fluorescence between $\mathrm{pH}$ 3.61 and pH 7.34 (Supplementary Fig. 5), and identified a particularly $\mathrm{pH}$-responsive variant we call mFAP_pH. 
a

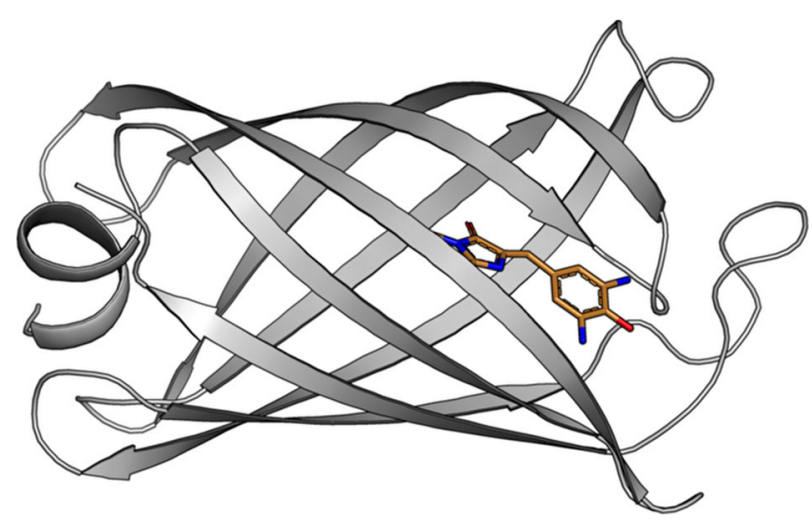

b<smiles>CC1=N/C(=C\c2cc(F)c(O)c(F)c2)C(=O)N1C</smiles>

1. DFHBI

C<smiles>CC1=N/C(=C\c2cc(F)c(O)c(F)c2)C(=O)N1CC(F)(F)F</smiles>

2. DFHBI-1T d

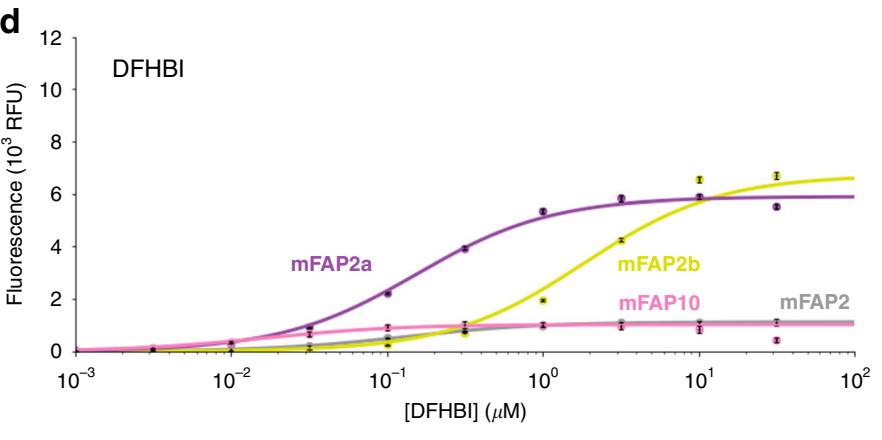

e

f
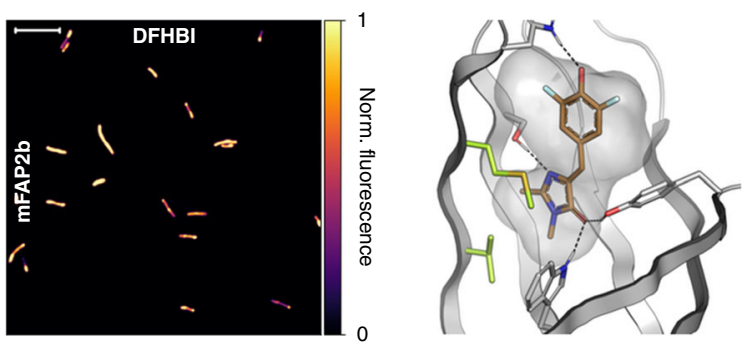

h

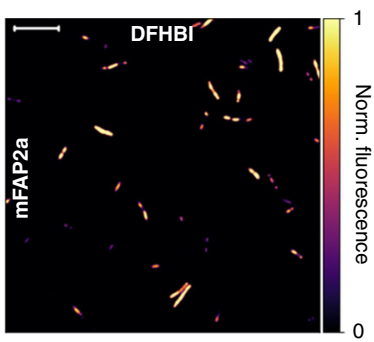

g
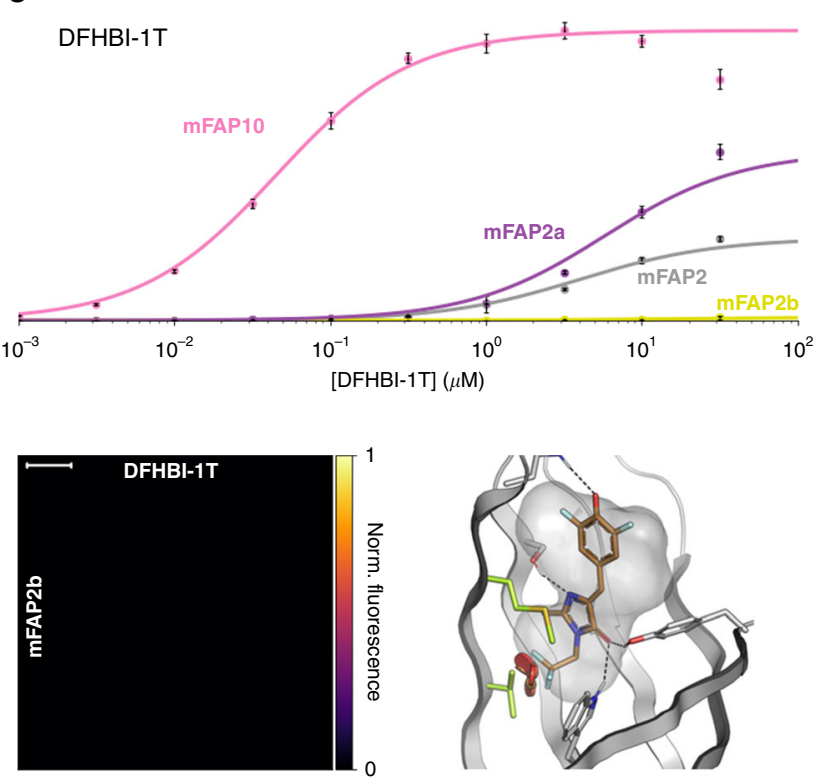

i
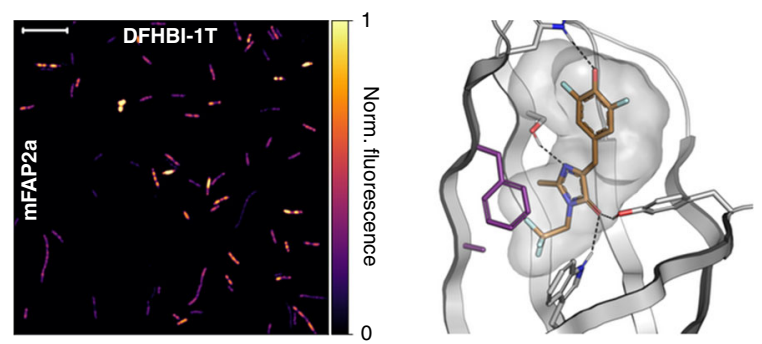

Fig. 1 Characterization of brighter and chromophore-specific mFAPs. a Computational model of de novo designed $\beta$-barrel variant mFAP2b showing protein backbone (cartoon) and bound DFHBI chromophore (sticks). $\mathbf{b}, \mathbf{c}$ Chemical structures of DFHBI and DFHBI-1T, respectively. $\mathbf{d}$, e In vitro titration of d DFHBI or e DFHBI-1T with mFAP2 (gray), mFAP2b (lime), mFAP2a (violet), and mFAP10 (pink) proteins. Error bars represent s.d. of the mean of eight technical replicates. Normalized means were fit to a single-binding site isotherm function using non-linear least squares fitting to obtain $K_{d}$ values (Table 1 ), and the fits scaled to the maximum mean relative fluorescence unit (RFU) values (lines). $\mathbf{f}-\mathbf{i}$ Each panel shows a representative image of the fluorescence signal emitted by $E$. coli cells expressing the indicated mFAP variant labeled with $10.0 \mu \mathrm{M}$ concentration of the indicated chromophore (left) and a zoom-in of the modeled-binding pocket of that mFAP variant bound to the chromophore (right). The images (left) are the pseudocolored normalized fluorescence intensity per pixel. Scale bars represent 10 microns. Imaging was independently repeated twice with similar results. The computational models (right) show the residues unique to mFAP2b (V13, M15) (lime sticks) or mFAP2a (A13, F15) (violet sticks). Intermolecular hydrogen bonds to the chromophore are shown as black dotted lines. Vacuum electrostatic contact potential around the chromophore is shown in a transparent gray surface. $\mathbf{f}$ mFAP2b with DFHBI, g mFAP2b with DFHBI-1T does not emit a detectable fluorescence signal because binding is precluded by steric clashes of DFHBI-1T with V13 (red cylinders), h mFAP2a with DFHBI, and i mFAP2a with DFHBI-1T. Source data is available for Fig. 1f-i. 


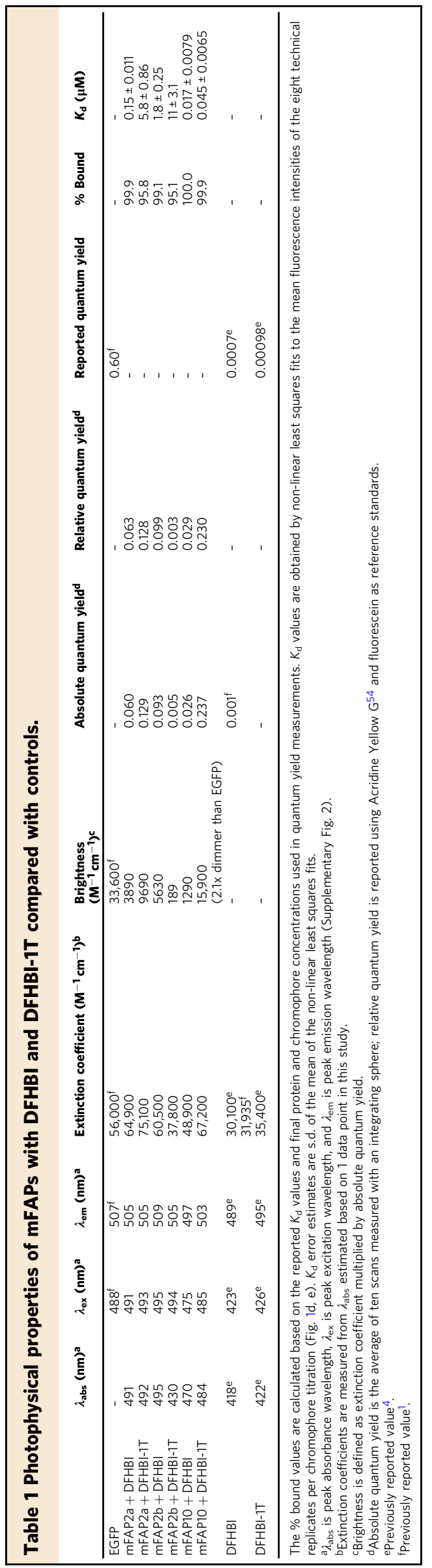

Computational models (Fig. 3b, c) suggest that the amino acid substitutions in mFAP_pH compared to mFAP2b (i.e., W27M and $\mathrm{W} 93 \mathrm{~F}$ ) improve $\mathrm{pH}$-responsiveness of the protein-DFHBI complex by increasing shape complementarity toward the protonated (phenolic) form of DFHBI by removing a hydrogen bond between the W27 indole ring and the DFHBI imidazolinone moiety, and by reducing net positive charge in the $\beta$-barrel core via removing a buried unsatisfied hydrogen bond donor in the indole ring of W93, resulting in a higher ratio of protein-bound phenolic DFHBI to phenolate DFHBI at low pH (Fig. 3f, g). The phenolic and phenolate forms of DFHBI had nearly equivalent affinities ( $K_{\mathrm{d}}$ values) for mFAP_pH of $\sim 190 \mathrm{nM}$ and $\sim 160 \mathrm{nM}$, respectively (Supplementary Fig. 5). The $\mathrm{pK}_{\mathrm{a}}$ of free, unbound DFHBI in solution ${ }^{5}$ is $\sim 5.4$, and we observe the same $\mathrm{pK}_{\mathrm{a}}$ for the mFAP_pH-DFHBI complex (Fig. 3h).

At peak excitation and emission wavelengths (Fig. 3d, e), mFAP_pH showed a marked $\sim 250$-fold-change in ratiometric fluorescence ( $F_{\text {ratio, }}$ see "Methods") from $\mathrm{pH} 8.38$ to $\mathrm{pH} 3.63$, compared with only a $\sim 34$-fold-change in $F_{\text {ratio }}$ from $\mathrm{pH} 8.38$ to $\mathrm{pH} 4.79$ for $\mathrm{pHRed}^{20}$ and a $\sim 3.5$-fold-change in $F_{\text {ratio }}$ from $\mathrm{pH}$ 8.83 to $\mathrm{pH} 5.14$ for pHluorin $2^{21}$ (Fig. $3 \mathrm{i}$ ). At low $\mathrm{pH}$, the $\beta$-barrel fold of mFAP_pH is more resistant to denaturation than those of pHRed and pHluorin2, and thus the mFAP_pH-DFHBI complex has a higher dynamic range for ratiometric fluorescence across the physiologically relevant $\mathrm{pH}$ range. Ratiometric fluorescence imaging of the mFAP_pH-DFHBI complex hence should enable real-time in situ quantification of $\mathrm{pH}$.

Incorporation of $\mathrm{Ca}^{2+}$-responsiveness. To enable the engineering of additional environmental responsiveness in mFAPs, we used Rosetta ${ }^{22,23}$ to de novo design 59 extensions of $\beta$-barrel loops 1, 3 and 7, and screened them for fluorescence after labeling with DFHBI. We identified five extended loop7 variants that maintain the $\beta$-barrel fold and are compatible with DFHBI binding (Supplementary Fig. 6). Using these extended loop sequences as linkers (see "Methods"), we grafted one EF-hand motif $^{24}$, one EF-hand domain ${ }^{25}$ (i.e., two EF-hand motifs), or calmodulin $^{26}$ (i.e., four EF-hand motifs) into loop7 of mFAP2b (Supplementary Fig. 7). Through DFHBI and $\mathrm{Ca}^{2+}$ titrations, we found that $\mathrm{Ca}^{2+}$ binding was allosterically coupled to DFHBI binding, with either positive ${ }^{7}$ or negative ${ }^{8}$ allosteric modulation of fluorescence (Fig. 4) depending only on the amino acid sequence of the linkers. As expected based on the cooperativity of $\mathrm{Ca}^{2+}$ binding to calmodulin'27,28, we found that as the number of grafted EF-hand motifs increases, so does the affinity for $\mathrm{Ca}^{2+}$ ions (Fig. 4f). While some existing fluorescent $\mathrm{Ca}^{2+}$ sensors harboring calmodulin such as GCaMP $6 \mathrm{f}^{29}$ are characterized by Hill coefficients of $\sim 2-3, \mathrm{Ca}^{2+}$-responsive mFAPs are characterized by Hill coefficients of $\sim 1$ (similar to previously reported ratiometric-pericam ${ }^{8}$, $\mathrm{CatchER}^{18}$, and $\mathrm{XCaMPs}^{30}$ ), suggesting that one $\mathrm{Ca}^{2+}$-binding site is allosterically coupled to chromophore affinity (Supplementary Table 2). Circular dichroism experiments showed that $\mathrm{Ca}^{2+}$ binding increases the $\alpha$-helical secondary structure, presumably in the calmodulin domain, and enhances thermostability for EF4n_mFAP2b harboring calmodulin (Supplementary Fig. 8).

Incorporation of the mFAP2a hydrophobic core amino acid substitutions (A13, F15) (Supplementary Fig. 9) increased $\mathrm{Ca}^{2+}$ affinity by up to 11.7 -fold for positively allosteric proteins and decreased $\mathrm{Ca}^{2+}$ affinity by up to 11.6 -fold for negatively allosteric proteins (Supplementary Table 2 and Supplementary Figs. 10, 11, $12,13)$. The substitutions increase the affinity of DFHBI binding (Fig. 1d), and DFHBI and $\mathrm{Ca}^{2+}$ titration data indicate thermodynamic coupling between DFHBI and $\mathrm{Ca}^{2+}$ binding (Supplementary Fig. 14 and Supplementary Note 1). Overall, the 

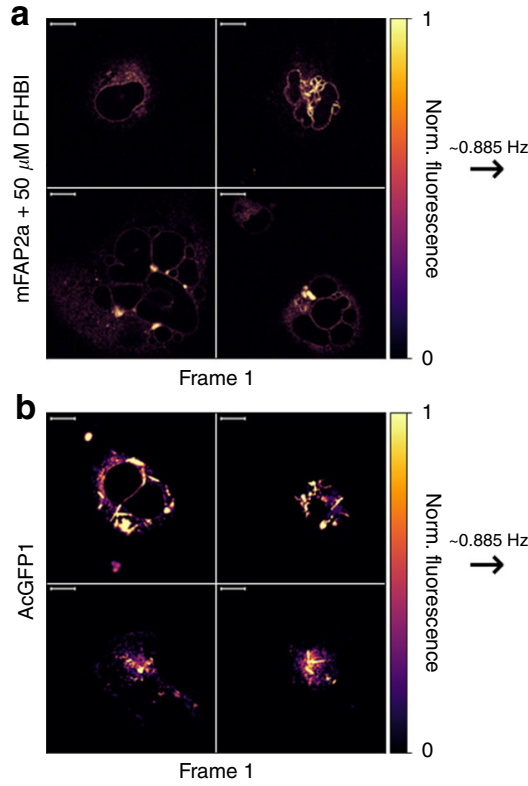

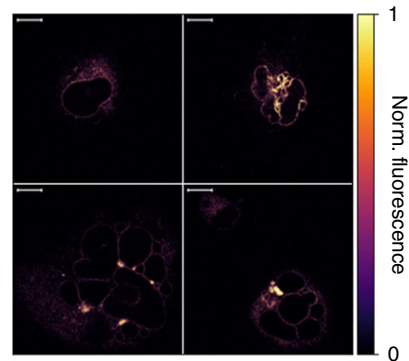

Frame 200

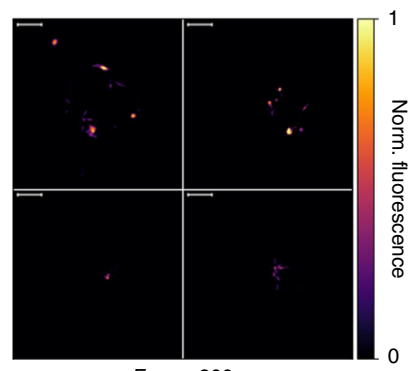

Frame 200

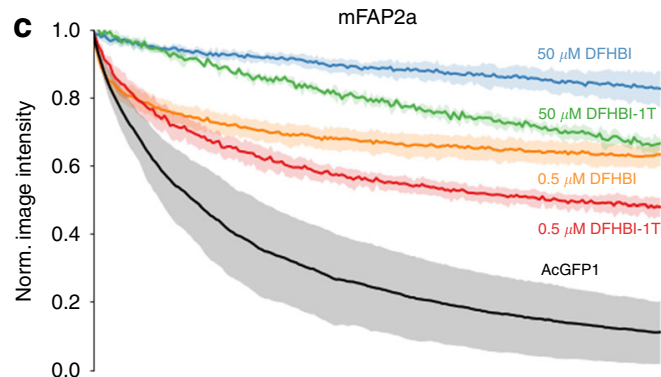

mFAP2b

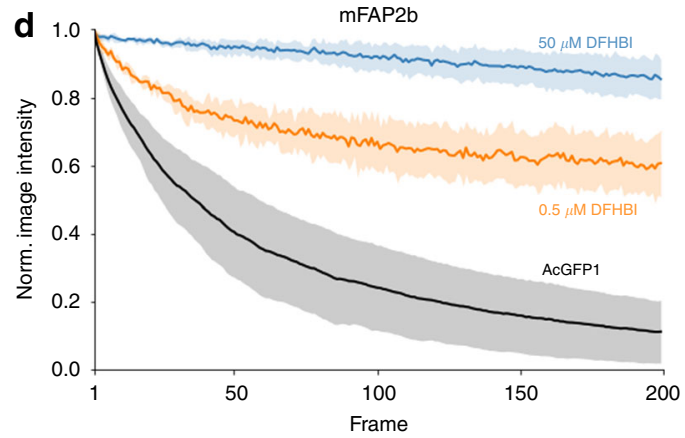

Fig. 2 Photostability of mFAPs compared to AcGFP1. a, b Normalized fluorescence intensity images for four regions of interest (ROIs) acquired under continuous wave imaging at $\sim 0.885 \mathrm{~Hz}\left(1.13 \mathrm{~s}\right.$ frame $\left.{ }^{-1}\right)$ shown for frame 1 (left) and frame 200 (right) of fixed COS-7 cells expressing endoplasmic reticulum-targeted a mFAP2a labeled with $50.0 \mu \mathrm{M}$ DFHBI compared to b AcGFP1. Scale bars represent 10 microns. c, d Means (lines) and s.d. of the means (shading) of the normalized summed pixel intensities of four ROls $(n=4)$ under continuous wave imaging ( $0.885 \mathrm{~Hz}$ ) for $\mathbf{c}$ mFAP2a labeled with $50.0 \mu \mathrm{M} \mathrm{DFHBI}$ ( 330-fold above $K_{d}$ ) (blue), $500 \mathrm{nM} \mathrm{DFHBI} \mathrm{(} \mathrm{3.3-fold} \mathrm{above} K_{d}$ ) (orange), $50.0 \mu \mathrm{M} \mathrm{DFHBI-1T}$ ( 8.6-fold above $K_{d}$ ) (green), or 500 nM DFHBI-1T ( 12-fold below $K_{d}$ ) (red) compared to AcGFP1 (black), and d mFAP2b labeled at $50.0 \mu \mathrm{M} \mathrm{DFHBI} \mathrm{(} \mathrm{28-fold} \mathrm{above} K_{\mathrm{d}}$ ) (blue) or 500 nM DFHBI ( 3.6-fold below $K_{d}$ ) (orange) compared to AcGFP1 (black). Source data is available for Fig. 2.

$\mathrm{Ca}^{2+}$-responsive mFAPs exhibit over 500-fold differences in affinity for $\mathrm{Ca}^{2+}$ (Supplementary Table 2), enabling the choice of a sensor with optimal fluorescence dynamic range in the anticipated $\mathrm{Ca}^{2+}$ concentration range under study.

To investigate the origin of the allosteric coupling between $\mathrm{Ca}^{2+}$ and DFHBI binding, we solved an X-ray crystal structure (Supplementary Table 3 ) of one of the positively allosteric $\mathrm{Ca}^{2+}$ responsive mFAPs harboring one EF-hand motif, EF1p2_mFAP2b, in complex with DFHBI and $\mathrm{Ca}^{2+}$ (Fig. 5a, b and Supplementary Fig. 15). The EF1p2_mFAP2b-DFHBI-Ca ${ }^{2+} \mathrm{co}^{-}$ crystal structure revealed the residue $\mathrm{K} 101$ from the $\mathrm{Ca}^{2+}$-bound EF-hand motif forms a hydrogen bond to the hydroxybenzylidene moiety of DFHBI, providing structural insight into the allosteric coupling mechanism between DFHBI and $\mathrm{Ca}^{2+}$ binding (Fig. $5 \mathrm{c}$ ). Indeed, the K101A lysine-to-alanine substitution in EF1p2_mFAP2b reduces DFHBI affinity $\sim 21$-fold in the presence of excess $\mathrm{Ca}^{2+}\left(K_{\mathrm{d}}^{+}\right)$, and $\mathrm{Ca}^{2+}$ affinity $\sim 29$-fold in the presence of excess DFHBI, compared with EF1p2_mFAP2b (Supplementary Fig. 16). This lysine residue is the second amino acid of the first EF-hand motif in all of the $\mathrm{Ca}^{2+}$-responsive mFAPs, suggesting it influences the allostery in each case. Molecular dynamics (MD) simulations starting from the X-ray crystal structure coordinates of EF1p2_mFAP2b in four conditions (apo, $\mathrm{Ca}^{2+}$-bound, DFHBI-bound and with both $\mathrm{Ca}^{2+}$ and DFHBI) suggest $\mathrm{Ca}^{2+}$ binding to loop7 shifts the free energy landscape towards the holo (fluorescently active) conformation even in the absence of DFHBI (Supplementary Fig. 17), suggesting a conformational selection $^{31,32}$ mechanism consistent with the experimentally observed allosteric coupling of DFHBI and $\mathrm{Ca}^{2+}$ binding (Supplementary Fig. 10).

To explore whether the $\mathrm{Ca}^{2+}$-responsive mFAPs could detect $\mathrm{Ca}^{2+}$ fluxes in mammalian cells, we first expressed a positively allosteric $\mathrm{Ca}^{2+}$-responsive mFAP harboring one EF-hand motif, EF1p_mFAP2b, in the extracellular matrix of HEK293 cells by fusion to an $\mathrm{N}$-terminal immunoglobulin $\kappa$-chain leader sequence secretion signal and a C-terminal transmembrane anchoring domain from platelet-derived growth factor receptor (PDGFR). To optimize detection sensitivity and photostability while compromising on fluorescence dynamic range (see Supplementary Note 1), we chose to label HEK293 cells with DFHBI concentrations at approximately the $\left(K_{\mathrm{d}}^{+} \cdot K_{\mathrm{d}}^{-}\right)^{1 / 2}$ for EF1p_mFAP2b, where $K_{\mathrm{d}}^{+}$and $K_{\mathrm{d}}^{-}$are the DFHBI $K_{\mathrm{d}}$ for the $\mathrm{Ca}^{2+}$-bound or $\mathrm{Ca}^{2+}$-free sensor, respectively (Supplementary Table 2). Titration of $\mathrm{Ca}^{2+}$ from 0 to $10 \mathrm{mM}$ final concentration under constant DFHBI concentration resulted in a fluorescence foldchange $\left(\frac{\Delta F}{F_{0}}\right)$ of $\sim 0.5$ (Fig. $5 \mathrm{~d}$ ). The fluorescence response was similar after photobleaching the cells, presumably due to the high-chromophore concentrations improving the photostability of mFAPs (Fig. 2).

Next, we expressed negatively allosteric $\mathrm{Ca}^{2+}$-responsive mFAPs containing two or four EF-hand motifs (EF2n_mFAP2a, EF4n_mFAP2b, and EF4n_mFAP2a) in the cytosol of HEK293 cells and stimulated $\mathrm{Ca}^{2+}$ release into the cytosol via endogenous muscarinic receptors ${ }^{19}$ by treatment with $100 \mu \mathrm{M}$ acetylcholine (ACh). As expected, $\mathrm{Ca}^{2+}$ release into the cytosol resulted in a decrease in fluorescence upon ACh stimulation (Fig. 5e and Table 2) with DFHBI concentrations at approximately $\left(K_{\mathrm{d}}^{+} \cdot K_{\mathrm{d}}^{-}\right)^{1 / 2}$, which balances detection sensitivity and photostability against fluorescence dynamic range (Supplementary Note 1). Compared to the positive control fluorescent $\mathrm{Ca}^{2+}$ sensor $^{29}$ GCaMP6f (Supplementary Fig. 18), the $\mathrm{Ca}^{2+}$-responsive mFAPs have lower fluorescence dynamic range at the DFHBI concentrations used, but are highly photostable (Fig. 5e, first $20 \mathrm{~s}$ ).

As the fluorescence of negatively allosteric $\mathrm{Ca}^{2+}$-responsive mFAPs increases when the $\mathrm{Ca}^{2+}$ concentration decreases, negatively allosteric $\mathrm{Ca}^{2+}$-responsive mFAPs enable reporting 


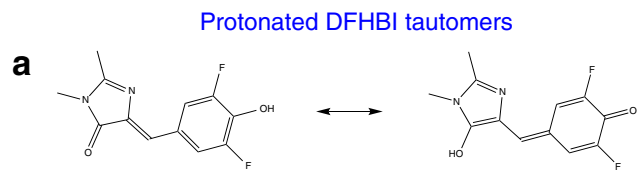

Deprotonated DFHBI resonance structures
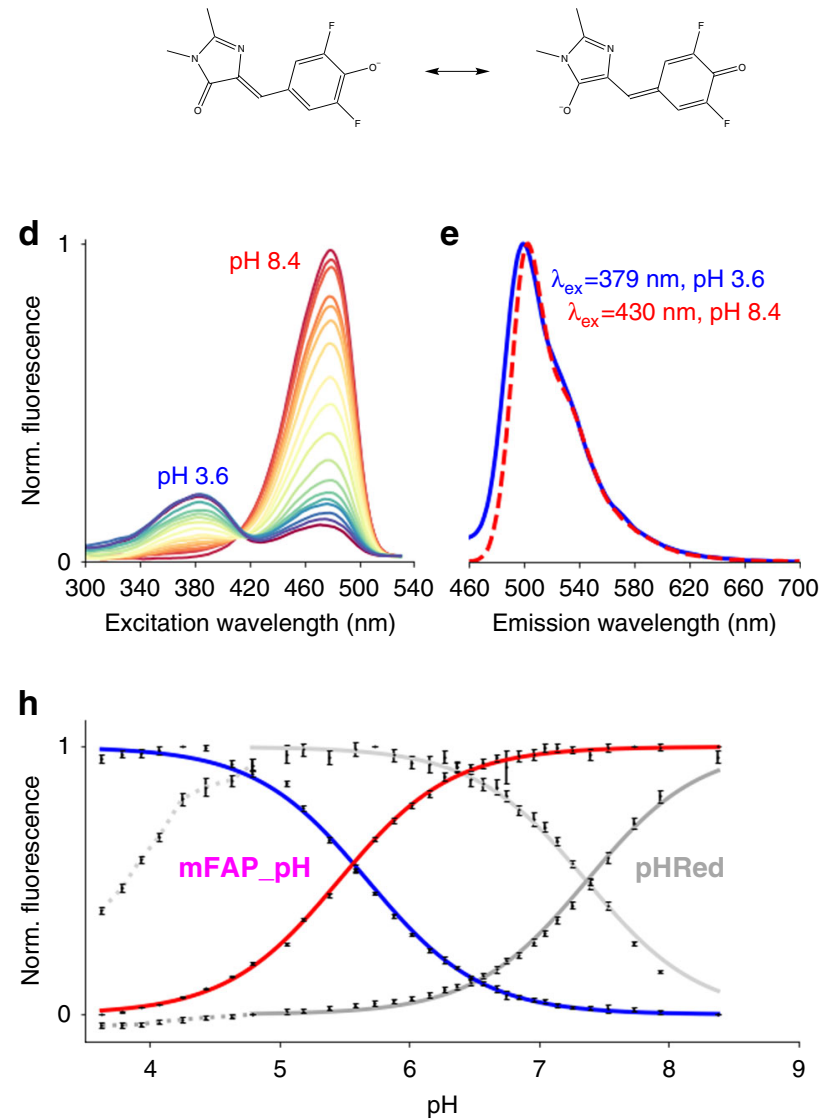

b
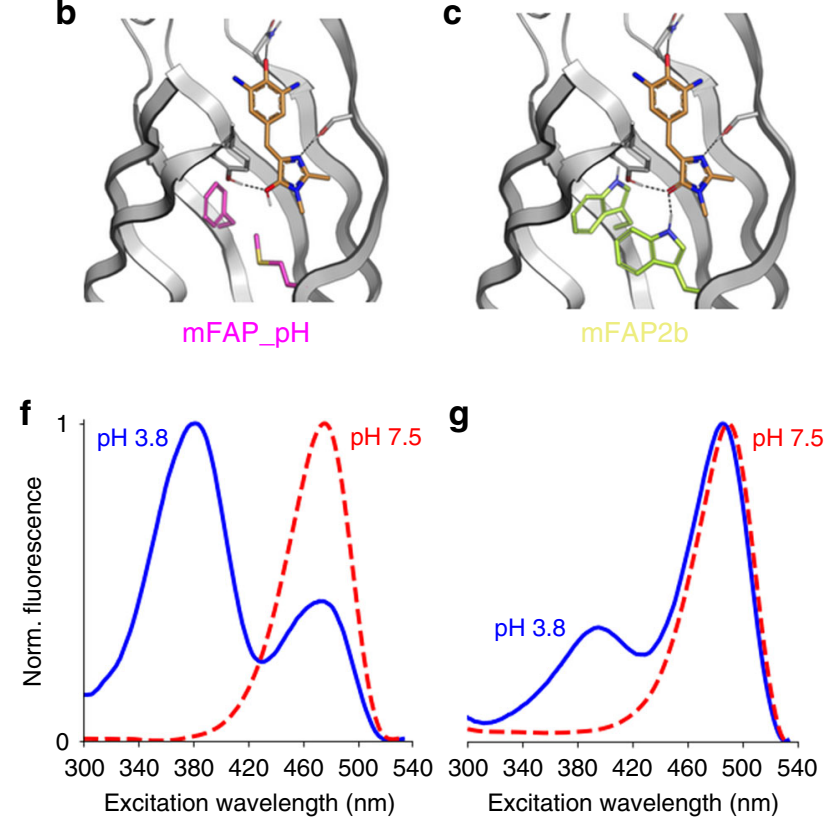

i

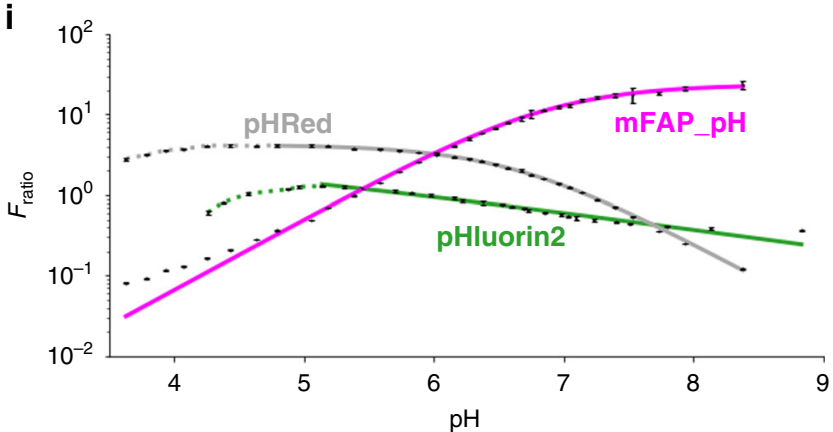

Fig. 3 Characterization of pH-responsive mFAP_pH. a-c Chemical basis of pH-responsiveness in mFAP_pH. a Chemical structures of protonated tautomers and deprotonated resonance structures of DFHBI. b, c Computational models showing the residues unique to b mFAP_pH (M27, F93) (magenta) and $\mathbf{c}$ mFAP2b (W27, W93) (lime). The arrangement of intermolecular hydrogen bonds (black dotted lines) in $\mathbf{b}$ the mFAP_pH-DFHBI complex permits binding to both the phenolate and phenolic (shown) forms of DFHBI whereas of $\mathbf{c}$ the mFAP2b-DFHBI complex only permits binding to the phenolate (shown) form of DFHBI. d Normalized fluorescence excitation spectra of the mFAP_pH-DFHBI complex for a pH titration between $\mathrm{pH} 3.6$ and $\mathrm{pH} 8.4$. e Normalized fluorescence emission spectra of the mFAP_pH-DFHBI complex at $\mathrm{pH} 3.6$ and pH 8.4. $\mathbf{f}, \mathbf{g}$ Fluorescence excitation spectra normalized to $\mathrm{pH}$ 3.8 and $\mathrm{pH} 7.5$ of $\mathbf{f} \mathrm{pH}$-responsive mFAP_pH-DFHBI complex and $\mathbf{g} \mathrm{pH}$-unresponsive mFAP2b-DFHBI complex. $\mathbf{h}$ Normalized mean $(n=3)$ fluorescence intensity from the pH titration of the mFAP_pH-DFHBI complex (blue and red) and previously reported pHRed (dark gray and light gray), showing fluorescence emission by exciting the blueshifted fluorescence excitation peak (blue and dark gray) and fluorescence emission by exciting the redshifted fluorescence excitation peak (red and light gray). i Ratiometric fluorescence ( $F_{\text {ratio }}$ ) from the pH titration of the mFAP_pH-DFHBI complex (magenta), pHRed (gray), and pHluorin2 (green). $\mathbf{h}, \mathbf{i}$ The means are fit to $\mathbf{h}$ a sigmoid or inverse sigmoid function or $\mathbf{i}$ a logistic function using non-linear least squares fitting (lines). The dotted lines indicate $\mathrm{pH}$ values at which the measured $F_{\text {ratio }}$ coincides with two different $\mathrm{pH}$ values, and therefore are not used in the fittings. Error bars represent the s.d. of the mean of three technical replicates.

$\mathrm{Ca}^{2+}$ effluxes from compartments as increases in fluorescence signal (similar to inverse-pericam ${ }^{8,33} \mathrm{Ca}^{2+}$-responsiveness). We expressed a negatively allosteric $\mathrm{Ca}^{2+}$-responsive mFAP containing one EF-hand motif, EF1n_mFAP2b, in the sarcoplasmic reticulum (SR) of cultured human induced pluripotent stem cell (hiPSC)-derived cardiomyocytes (CMs), as existing $\mathrm{Ca}^{2+}$-responsive fluorescent protein sensors targeted to the SR report $\mathrm{Ca}^{2+}$ effluxes as decreases in fluorescence signal ${ }^{34,35}$. Again optimizing photostability while compromising on fluorescence fold-change, we chose to label CMs with a DFHBI concentration at approximately $\left(K_{\mathrm{d}}^{+} \cdot K_{\mathrm{d}}^{-}\right)^{1 / 2}$ for EF1n_mFAP2b (Supplementary Table 2). $\mathrm{Ca}^{2+}$ transients in the SR during cardiac contraction cycling were detectable with high photostability (Supplementary
Fig. 19). Labeling CMs with DFHBI concentrations at approximately $\frac{K_{\mathrm{d}}^{-}}{2}$ for EF1n_mFAP2b (Supplementary Table 2) resulted in robustly detectable $\mathrm{Ca}^{2+}$ transients during cardiac contraction cycling and moderate photostability using $\sim 3$-fold higher laser power density (Fig. 5f). The image acquisition rate in these experiments was $\sim 16.7 \mathrm{~Hz}\left(60 \mathrm{~ms}\right.$ frame $\left.^{-1}\right)$, suggesting that EF1n_mFAP2b responds to $\mathrm{Ca}^{2+}$ fluxes in less than $60 \mathrm{~ms}$. Temporal analysis averaged over 20 cardiac contraction cycles revealed a $96 \pm 45 \mathrm{~ms}$ (mean \pm s.d.) lag time between peak SR fluorescence and peak cardiac contraction, indicating that endogenous SR $\mathrm{Ca}^{2+}$ signaling precedes cardiomyocyte contractile motion. Inhibition of $\mathrm{Ca}^{2+}$ reuptake into the SR by treatment with cyclopiazonic acid (CPA) resulted in a sustained increase in 
a

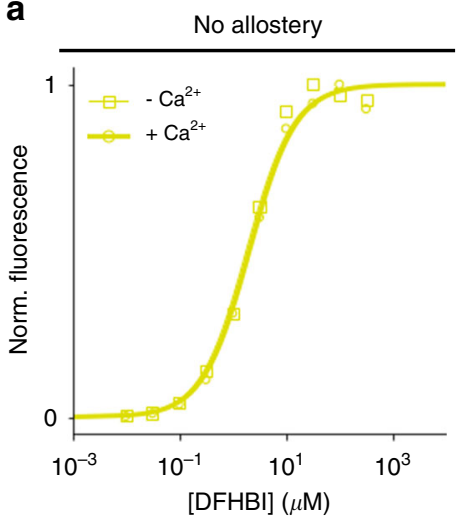

d

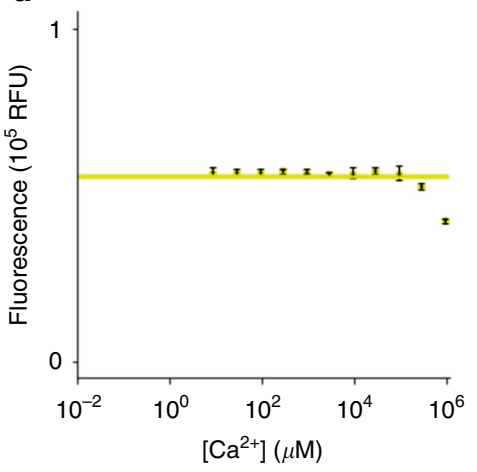

b

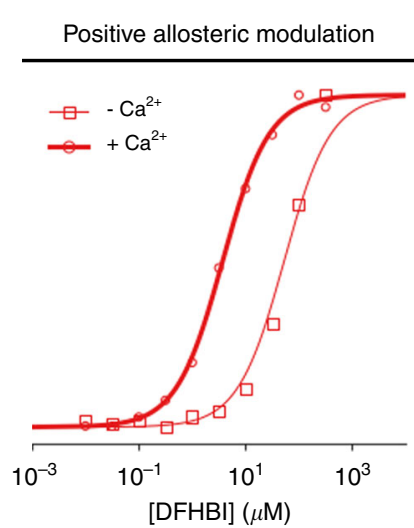

e

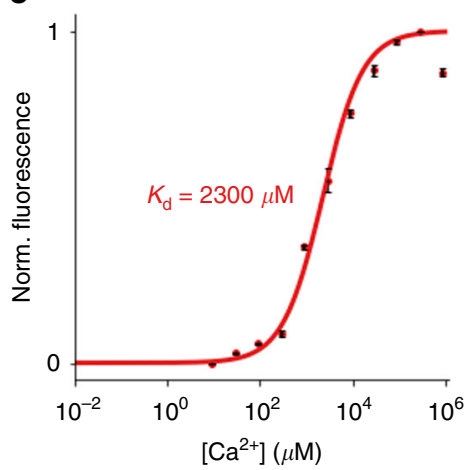

c

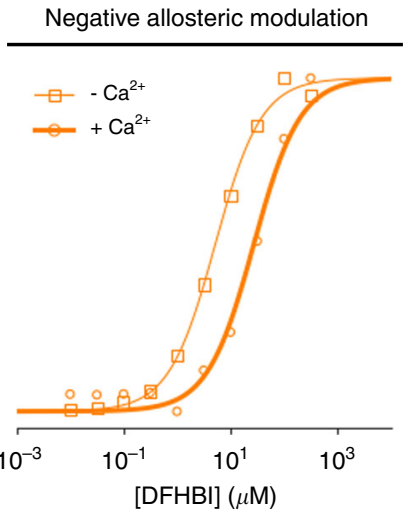

f

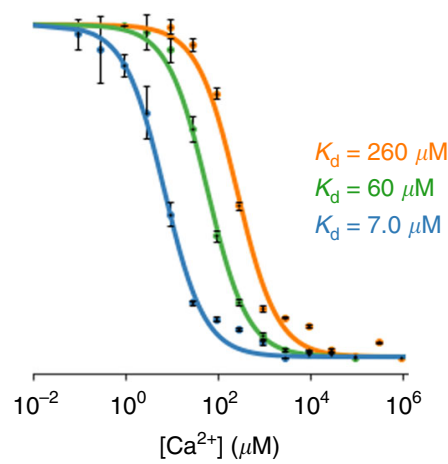

Fig. 4 In vitro characterization of $\mathbf{C a}^{2+}{ }^{2}$-responsive mFAPs. a-c DFHBI titration in the absence of $\mathrm{Ca}^{2+}$ (squares) and presence of $\mathrm{Ca}^{2+}(\mathrm{circles})$. a For mFAP2b, $\mathrm{Ca}^{2+}$ does not affect DFHBI binding. b For EF1p2_mFAP2b, binding of $\mathrm{Ca}^{2+}$ and DFHBI exhibit positive allostery. c For EF1n_mFAP2b, binding of $\mathrm{Ca}^{2+}$ and DFHBI exhibit negative allostery. $\mathbf{a}-\mathbf{c}$ Normalized fluorescence intensities $(n=1)$ are fit to a sigmoid function using non-linear least squares fitting (lines). $\mathbf{d}-\mathbf{f} \mathrm{Ca}^{2+}$ titrations with excess DFHBI concentration compared to protein concentration. $\mathbf{d}$ Unnormalized mean fluorescence intensities of mFAP2b

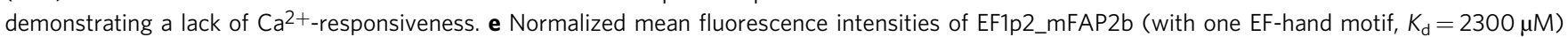
demonstrating positive allostery between DFHBI and $\mathrm{Ca}^{2+}$ binding. $\mathrm{C}^{2+}{ }^{2+}$-responsiveness is dependent on the number of EF-hand motifs inserted into loop7, as exemplified by the normalized mean fluorescence intensities of EF1n_mFAP2b (with one EF-hand motif, $K_{d}=260 \mu M$ ), EF2n_mFAP2b (with two EF-hand motifs, $K_{d}=60 \mu \mathrm{M}$ ), and EF4n_mFAP2b (with four EF-hand motifs, $K_{d}=7.0 \mu \mathrm{M}$ ), demonstrating negative allostery between DFHBI and Ca2+ binding. d-f Error bars represent the s.d. of the mean of three technical replicates. The means $(n=3)$ are fit to a $\mathbf{d}$ constant function, or $\mathbf{e}$ sigmoid or $\mathbf{f}$ inverse sigmoid function with Hill coefficients of 1 , using non-linear least squares fitting (lines) to obtain $K_{d}$ values (Supplementary Table 2).

fluorescence in the SR consistent with inhibition of SERCA pumps $^{34,36}$ (Supplementary Fig. 19).

Split fluorescence-activating proteins. We next sought to design bipartite split fluorogenic sensors ${ }^{13}$ from mFAPs by creating split points in the $\beta$-hairpins and loop7 of the mFAP2a scaffold. With eight $\beta$-strands ${ }^{1}$ per $\beta$-barrel, there are seven possible bipartite split mFAPs (Supplementary Fig. 20). As the split mFAP fragments would have solvent-exposed hydrophobic patches that could hamper solubility, we initially tagged split mFAP fragments to maltose-binding protein (MBP) to improve soluble expression levels. $\beta$-barrel complementation assays in excess DFHBI-1T showed that split mFAP fragments $\mathrm{m} 12$ and $\mathrm{m} 38$ displayed the highest fluorescence activation above background, with 7.34-fold higher mean fluorescence intensity over mean background fluorescence intensity. After background subtraction, the brightest fragment combination, $\mathrm{m} 12$ and $\mathrm{m} 38$, had 184-fold higher mean fluorescence intensity than the dimmest fragment combination, $\mathrm{m} 1$ and $\mathrm{m} 28$. Differences in the fluorescence excitation spectra of the fluorescently active $\beta$-barrel complexes in excess DFHBI-1T suggest that bipartite split mFAPs stabilize the fluorescently active cis-planar conformation of DFHBI-1T in slightly different chromophore environments (Supplementary Fig. 20).

Titrations of MBP-tagged split mFAP fragments into their complementary MBP-tagged split mFAP fragments in excess DFHBI-1T resulted in reconstitution of fluorescence at highprotein concentrations, but the signal did not plateau even at the highest concentrations tested. The estimated split mFAP fragment dissociation constants ( $K_{\mathrm{d}}$ values) are $\geq 281 \mu \mathrm{M}$ for $\mathrm{m} 12$ and $\mathrm{m} 38, \geq 22.0 \mu \mathrm{M}$ for $\mathrm{m} 14$ and $\mathrm{m} 58, \geq 232 \mu \mathrm{M}$ for $\mathrm{m} 16$ and $\mathrm{m} 78$, and $\geq 354 \mu \mathrm{M}$ for $\mathrm{m} 17$ and $\mathrm{m} 8$ (Supplementary Fig. 20). In contrast, when we fused complementary split mFAP fragments to BCL2 family member proteins and high affinity $\left(K_{\mathrm{d}} \simeq 1 \mathrm{nM}\right)$ designed binding partners ${ }^{37}$ (Fig. 6a), the fluorescence increased linearly until reaching a plateau at equimolar concentrations of complementary split mFAP fragments (Fig. 6b).

To assess whether split mFAPs could be used for real-time monitoring of protein-protein association, we pre-incubated equimolar BCLXL_m58 with unfused aBCLXL in excess DFHBI$1 \mathrm{~T}$ to pre-assemble non-fluorescent BCLXL_m58-aBCLXL complex. Upon addition of equimolar m14_aBCLXL (or buffer as a negative control), the fluorescence increased as m14_aBCLXL competed with unfused aBCLXL for the BCLXL-binding cleft of BCLXL_m58, resulting in assembly of the m14-m58 complex, 
a

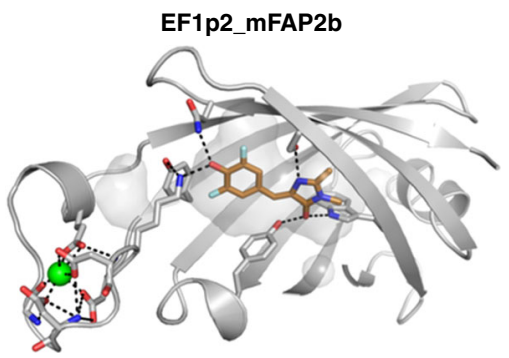

b

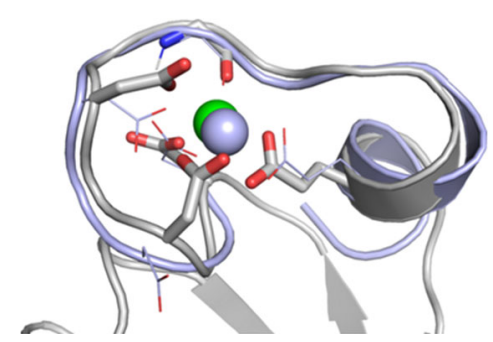

C

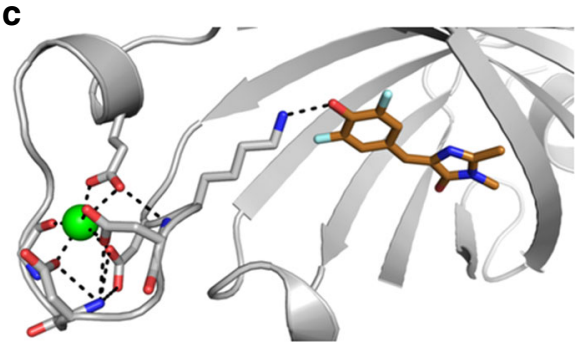

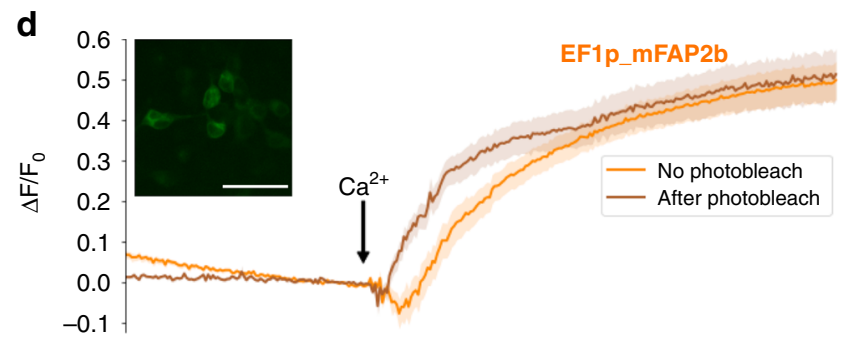

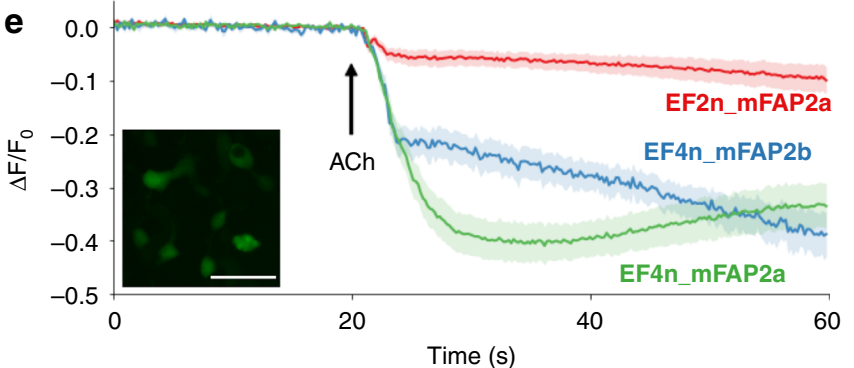

f

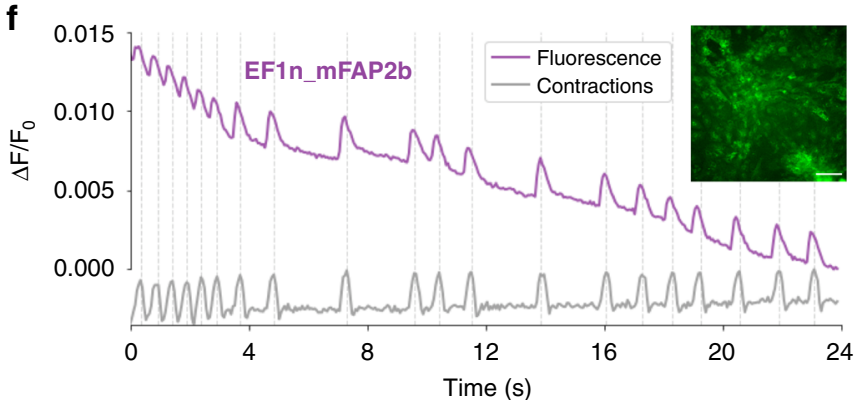

Fig. 5 Structural and in cell characterization of $\mathbf{C a}^{\mathbf{2}+}$-responsive mFAPs. a-c X-ray co-crystal structure of EF1p2_mFAP2b bound to DFHBI and Ca ${ }^{2+}$ at 2.1 $\AA$ resolution. a Structure of monomer with $\mathrm{Ca}^{2+}$ (green sphere) and DFHBI (copper sticks) bound, with protein side-chains (gray sticks) forming first- and second-shell hydrogen bonds with $\mathrm{Ca}^{2+}$ and DFHBI (black dotted lines), and vacuum electrostatic contact potential around DFHBI (transparent gray surface). b Aligned is the nuclear magnetic resonance (NMR) solution structure from PDB accession code 1 NKF (violet cartoon and lines) with La ${ }^{3+}$ ion bound (violet sphere) having the closest $\mathrm{C}_{\alpha}-\mathrm{C}_{\alpha}$ root mean square deviation (2.19 $\AA$ ) to the structure of the grafted EF-hand motif (gray sticks and cartoon) with bound Ca2+ ion (green sphere). c Co-crystal structure of EF-hand motif residues (gray sticks) reveals that residue K101 from the EF-hand motif directly hydrogen bonds (black dotted lines) with DFHBI, suggesting an allosteric coupling mechanism between Ca ${ }^{2+}$ (green sphere) and DFHBI (copper sticks) binding. d Fluorescence imaging of $\mathrm{Ca}^{2+}$ titrations (beginning at arrow) of live HEK293 cells expressing extracellular EF1p_mFAP2b, demonstrating positive allostery in cyto. Average fluorescence fold-change (lines) and s.e.m. (shading) is shown for regions of interest (ROIs) surrounding single cells without photobleaching (orange; $n=15$ ) or after a photobleaching challenge (dark orange; $n=15$ ) from three technical replicates. e Fluorescence imaging of acetylcholine (ACh) stimulations (added at arrow) of live HEK293 cells expressing cytosolic either EF2n_mFAP2a (red), EF4n_mFAP2b (blue), or EF4n_mFAP2a (green), demonstrating negative allostery in cyto. Average fluorescence fold-change (lines) and s.e.m. (shading) are shown for ROls surrounding single cells expressing EF2n_mFAP2a ( $n=$ 15), EF4n_mFAP2b $(n=10)$, or EF4n_mFAP2a $(n=15)$ from three technical replicates (Table 2). f Fluorescence imaging of live human induced pluripotent stem cell-derived cardiomyocytes expressing sarcoplasmic reticulum-targeted EF1n_mFAP2b (violet) labeled with DFHBI at approximately $\frac{K_{d}^{-}}{2}$

(Supplementary Table 2) showing whole field of view normalized fluorescence fold-change, demonstrating negative allostery in cyto. The normalized average of three ROI traces in the fluorescence channel (gray) indicate peak cardiac contraction frames (dotted lines). d-f Representative whole field of view pseudocolored maximum intensity z-axis projections. Scale bars represent $\mathbf{d}$, e 50 and $\mathbf{f} 100$ microns. Source data is available for Fig. $5 \mathrm{~d}$-f.

which activates the fluorescence of DFHBI-1T (Fig. 6c, d). The reaction evolved analogously for BFL1-aBFL1 and BCL2-aBCL2 cognate-binding partners. Different peak fluorescence foldchanges observed amongst split mFAP fusions to BCLXL-aBCLXL, BCL2-aBCL2, and BFL1-aBFL1 complexes suggest that the molecular geometry of the heterodimer interaction affects the brightness of the assembled $\beta$-barrel complex. Fluorescence excitation spectra revealed a prominent peak in fluorescence excitation wavelength at $488 \mathrm{~nm}$ upon combining split mFAP fragments compared to buffer negative controls (Supplementary Fig. 21).

To assess whether split mFAPs could be used for real-time monitoring of protein-protein dissociation, we pre-incubated
BCL2_m58 with equimolar m14_aBFL1 in excess DFHBI-1T to pre-assemble fluorescent complexes. As the non-cognate BCL2-aBFL1 complex has a dissociation constant $\left(K_{\mathrm{d}}\right)$ of $320 \pm$ $40 \mathrm{nM}$, the cognate BCL2-aBCL2 complex has a $K_{\mathrm{d}}$ of $0.8 \pm$ $0.5 \mathrm{nM}^{37}$, and aBFL1 and aBCL2 interact with the same binding cleft of BCL2, aBCL2 should outcompete aBFL1 for binding to BCL2 (Fig. 6e). Indeed, titration of aBCL2 into pre-assembled BCL2_m58-m14_aBFL1 complex in excess DFHBI-1T resulted in an aBCL2 concentration-dependent decrease in fluorescence (Fig. 6f). Fluorescence excitation spectra showed the disappearance of the fluorescence excitation peak at $488 \mathrm{~nm}$ wavelength consistent with chromophore unbinding and deactivation of fluorescence upon split mFAP fragment disassembly (Supplementary Fig. 21). 
Table 2 Fluorescence response to acetylcholine stimulation of HEK293 cells expressing cytosolic $\mathrm{Ca}^{2+}{ }_{\text {-responsive mFAPs or }}$ GCaMP6f.

\begin{tabular}{lcccc} 
Sensor & Peak $\left|\frac{\Delta \boldsymbol{F}}{\boldsymbol{F}_{\mathbf{0}}}\right|$ & [DFHBI] $(\boldsymbol{\mu M} \mathbf{M})$ & Regions of interest & $\begin{array}{c}\text { Fluorescence response to } \\
\text { increased }\left[\mathbf{C a}^{\mathbf{2}+} \mathbf{]}\right.\end{array}$ \\
\hline EF2n_mFAP2a & $0.12 \pm 0.092$ & 20.0 & 15 & Negative \\
EF4n_mFAP2b & $0.42 \pm 0.14$ & 43.3 & 10 & Negative \\
EF4n_mFAP2a & $0.46 \pm 0.12$ & 43.3 & 15 & Negative \\
GCaMP6f & $11 \pm 1.8$ & 0.00 & 12 & Positive
\end{tabular}

The mean and s.d. of the mean of peak absolute values of the fluorescence fold-change (peak $\left.\left|\frac{\Delta F}{F_{0}}\right|\right)$ upon acetylcholine (ACh) stimulation over the indicated number of regions of interest surrounding single cells (3 technical replicates per sensor; Fig. 5e and Supplementary Fig. 18).

Circular permutation. To further explore the range of possibilities for mFAP-based sensors, we circularly permuted mFAPs (cpmFAPs) using Rosetta and the split points from the four brightest bipartite split mFAPs. The brightest cpmFAP tested, cp35-34_mFAP2a_12, has a de novo designed $\alpha$-helical linker and displayed $\sim 93 \%$ of the fluorescence intensity of mFAP2a at equimolar concentration and excess DFHBI-1T (Supplementary Fig. 22). Size-exclusion chromatography with multi-angle light scattering showed cp35-34_mFAP2a_12 to be monomeric (Supplementary Fig. 23).

\section{Discussion}

We have demonstrated that the functionality and brightness of mFAPs can be readily extended by structure-based design and engineering. It should be emphasized, however, that the engineering of useful fluorogenic sensors based on mFAPs is still in its early days-the mFAPs were first described in September $2018^{1}$. Currently, existing fluorescent protein-based sensors still have numerous advantages over mFAPs in brightness (e.g., EGFP is $\sim 2.1$-fold brighter than the mFAP10-DFHBI-1T complex) for applications involving single molecule localization microscopy ${ }^{15,38}$, fluorescence spectral diversity ${ }^{39}$, higher $\mathrm{Ca}^{2+}$ affinity $^{7}$, and higher fluorescence dynamic range ${ }^{29}$, as in selflabeling chemigenetic indicators ${ }^{40-42}$. These sophisticated reporters and sensors reflect decades of work by many groups; we hope this report will stimulate exploration of de novo designed fluorescence-activating proteins. With further optimization using both selection and computational design methodologies, there is likely considerable room for improvement of brightness, photostability, $\mathrm{pH}$-responsiveness, and $\mathrm{Ca}^{2+}$-responsiveness.

At this stage, the possible advantages of de novo designed mFAP sensors over existing fluorescent protein-based reporters are listed in Table 3. Two notable advantages of split mFAPs over existing split GFP-based approaches for monitoring transient protein-protein interactions are the rapid activation of fluorescence upon assembly of split mFAP fragments that enables tracking of protein-protein association, and the rapid deactivation of fluorescence upon disassembly of split mFAP fragments that enables tracking of protein-protein dissociation (similar to splitFAST but with $\sim 2$ orders of magnitude lower fragment affinities). mFAPs can activate the fluorescence of DFHBI-derived chromophores with emission spectra in different color ranges, as illustrated by the activation of $\mathrm{DFHO}^{5}$ fluorescence by mFAP3. mFAPs can be used as modular fluorogenic optical sensors for detection and quantification of other small-molecules, ions, or proteins by insertion of their respective binding peptides into the loops of mFAPs without circularly permuting the mFAPs, as in construction of $\mathrm{Ca}^{2+}$-responsive mFAPs. The cpmFAPs enable design of modular fluorogenic sensors by fusing analyte binding peptides (e.g., calmodulin and M13) directly to the juxtaposed termini or within $\beta$-sheets, as in construction of GCaMP19,29. More generally, as brighter and more photostable fluorogenic compounds are developed, the methodologies described herein should be readily applicable to creating protein-based fluorogenic optical sensors from binders to these compounds.

\section{Methods}

Design of brighter and pH-responsive mFAPs. A previously described ${ }^{1}$ mFAP2 computational design model was used as a template for manual selection and design of mutable residues using Rosetta ${ }^{23,43}$ macromolecular modeling software. Guided by the previously generated deep mutational scanning maps ${ }^{1}$ of stability and fluorescence of b11L5F, we constructed three mFAP2 mutational variants mFAP2(P50T,S52V), mFAP2(S52T), and mFAP2(P50T,S52V,G100D) that were expected to improve the stability of the protein while also aiding crystallization. Circular dichroism in the absence of DFHBI revealed that mFAP2(P50T,S52V), hereafter called mFAP2.1, demonstrated improved stability at pH 2.93 (Supplementary Fig. 1b, c) and higher fluorescence in the presence of DFHBI at pH 3.66 (Supplementary Fig. 1d, e) compared to mFAP2, consistent with improved binding of the phenolic form of DFHBI to the stabilized protein. A minimal site-directed mutagenesis (SDM) library (Supplementary Data 3) was generated at 15 residue positions on mFAP2.1 encoding mutations hypothesized to improve the fluorescence ratio fold-change from low to high $\mathrm{pH}$, increase DFHBI affinity, and reduce conformational flexibility of the loop connecting the seventh and eighth $\beta$-strands (known as loop7) juxtaposing the DFHBI-binding pocket.

Fluorescence screening of the SDM library at $\mathrm{pH} 3.66$ and $\mathrm{pH} 7.36$ revealed that the most pH-responsive mutant mFAP2.1(T50P), also known as mFAP2.2,

demonstrated $\sim 1.3$-fold higher fluorescence ratio fold-change from $\mathrm{pH} 3.66-7.36$ than mFAP2.1 (Supplementary Fig. 1f). Subsequently, two independent combinatorial libraries were generated from mFAP2.2: one at five positions aimed at increasing loop7 rigidity (Supplementary Data 4), and another at eight positions aimed at optimizing hydrophobic packing of residues in the hydrophobic $\beta$-barrel core (Supplementary Data 5). The brightest variant from the first library mFAP2.2 (A100E,G101N,N102D,T104H), hereafter known as mFAP2.3, and the brightest variant of the second library mFAP2.2(M27W,V39I,V57A,F93W), hereafter known as $\mathrm{mFAP} 2.4$, showed an increase in fluorescence intensity from the phenolate form of DFHBI of $\sim 1.1$-fold and $\sim 3.4$-fold from mFAP2.2 at $\mathrm{pH} 7.36$, respectively (Supplementary Fig. 1g,h). The mutations producing mFAP2.3 and mFAP2.4 were combined into one scaffold generating mFAP2.5. A single mutation (V67I) was identified by screening a combinatorial library (Supplementary Data 6) of mutations at 7 positions aimed at packing more methyl groups into the hydrophobic $\beta$-barrel core of mFAP2.5. The protein (Fig. 1a), referred to as mFAP2b ("b" for bright), is 1.2-fold brighter than mFAP2.5 and $\sim 1.3$-fold brighter than mFAP2.4 at neutral $\mathrm{pH}$ (Supplementary Fig. 1i). It was demonstrated that mFAP2b had $\sim 10$-fold weaker affinity for DFHBI than the initial mFAP2 design.

Another combinatorial library (Supplementary Data 7) was generated at five positions of mFAP2b aimed at increasing affinity for the deprotonated state of DFHBI without compromising fluorescence intensity by packing both aromatic and aliphatic residues in the hydrophobic $\beta$-barrel core of mFAP2b. Screening for fluorescence intensity of the phenolate state of DFHBI using a relatively lowDFHBI concentration ( $555 \mathrm{nM}$ ) resulted in mFAP2b(V13A,M15F), known as mFAP2a ("a" for affinity). mFAP2a displayed $\sim 1.3$-fold brighter fluorescence than mFAP2b at low-DFHBI concentration (Supplementary Fig. 1j). Aiming to further improve packing of methyl groups in the hydrophobic $\beta$-barrel core to increase the fluorescence intensity of the phenolate state of DFHBI at neutral $\mathrm{pH}$ while accommodating the interesting geometry of the Y71W mutation, a combinatorial library (Supplementary Data 8) was constructed at three positions of mFAP2a, resulting in designs mFAP3 through mFAP8 (Supplementary Table 1). However, these mutants were dimmer and demonstrated lower expression levels than mFAP2b or mFAP2a (Supplementary Table 1), although mFAP3 showed to be the brightest DFHO-binding variant (mFAP3 with $10.0 \mu \mathrm{M}$ DFHO is $\sim 10$-fold dimmer than mFAP2b with $10.0 \mu \mathrm{M}$ DFHBI). In order to increase the fluorescence brightness of mFAP2a with DFHBI-1T, a final combinatorial library was constructed at six positions of mFAP2a (Supplementary Data 9) by mutating aliphatic and aromatic residues in the chromophore-binding pocket of mFAP2a 
a

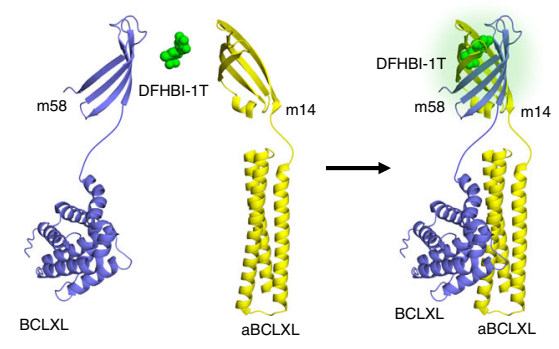

C

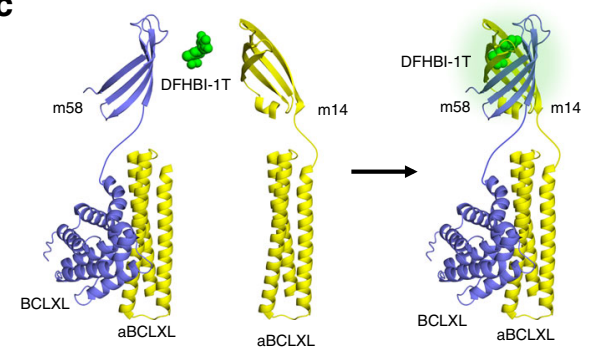

b

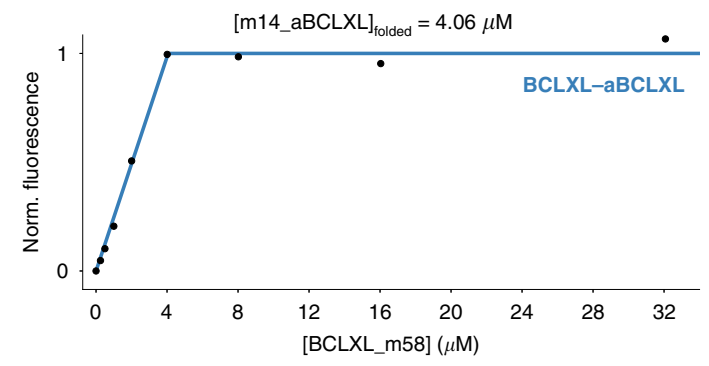

d

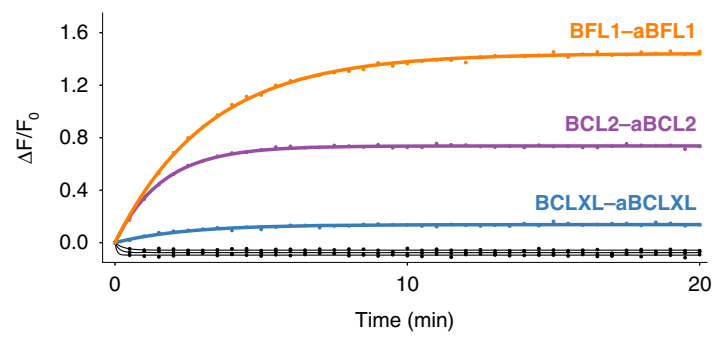

f

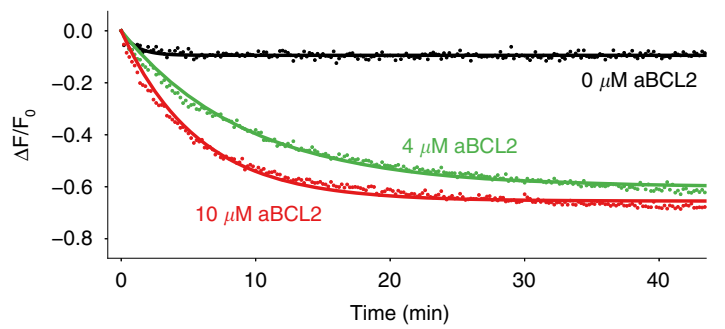

Fig. 6 Assembly and disassembly of bipartite split mFAP fragments $\mathbf{m 1 4}$ and $\mathbf{~ m 5 8 . ~ a - d ~ A s s e m b l y ~ o f ~ s p l i t ~ m F A P ~ f r a g m e n t s . ~ a ~ A s s o c i a t i o n ~ m o d e l ~ i n ~ w h i c h ~}$ $B C L X L$ is fused to $\mathrm{m} 58$ (BCLXL_m58) (violet cartoon), aBCLXL is fused to m14 (m14_aBCLXL) (yellow cartoon), and fluorescence of DFHBI-1T (green spheres) is activated upon association (arrow) of BCLXL_m58 and m14_aBCLXL. b Normalized fluorescence intensity (points) of BCLXL_m58 titration into a constant m14_aBCLXL concentration in excess DFHBI-1T after reaching equilibrium, showing the fit to a bimolecular association model (line) using non-linear least squares fitting. c Split mFAP competitor pre-incubation model in which fluorescence of DFHBI-1T (green spheres) is activated upon competition (arrow) of m14_aBCLXL with unfused aBCLXL (yellow cartoons) for the BCLXL-binding cleft of BCLXL_m58 (violet cartoon) (the reaction evolves analogously for BFL1-aBFL1 and BCL2-aBCL2 cognate-binding partners). d Temporal evolution of fluorescence fold-change in excess DFHBI-1T upon ( $n=1$ ) addition of equimolar m14_aBFL1 (orange points) or buffer (black points) to pre-incubated equimolar BFL1_m58 and aBFL1, addition of equimolar m14_aBCL2 (violet points) or buffer (black points) to pre-incubated equimolar BCL2_m58 and aBCL2, and addition of equimolar m14_aBCLXL (blue points) or buffer (black points) to pre-incubated equimolar BCLXL_m58 and aBCLXL, showing the fits to a monophasic exponential function (lines) using non-linear least squares fitting. e, f Disassembly of split mFAP fragments. e Pre-assembled split mFAP competition model in which BCL2 is fused to m58 (BCL2_m58) (violet cartoon), aBFL1 is fused to m14 (m14_aBFL1) (orange cartoon), and fluorescence of DFHBI-1T (green spheres) is activated before unfused aBCL2 (yellow cartoon) competes with m14_aBFL1 for the BCL2-binding cleft of BCL2_m58 (arrow), resulting in fluorescence deactivation. $\mathbf{f}$ Temporal evolution of fluorescence fold-change in excess DFHBI-1T of pre-incubated equimolar BCL2_m58 and m14_aBFL1 at 2.00 $\mu \mathrm{M}$ final concentrations with unfused aBCL2 titrated in at $(n=1) 0 \mu \mathrm{M}$ (black points), $4.00 \mu \mathrm{M}$ (green points), and $10.0 \mu \mathrm{M}$ (red points) final concentrations, showing the fits to a monophasic exponential function (lines) using non-linear least squares fitting. Source data is available for Fig. 6b, d, f.

juxtaposing DFHBI-1T in computational models. Screening for fluorescence intensity of DFHBI-1T using a low-DFHBI-1T concentration $(250 \mathrm{nM})$ resulted in mFAP2a(W27I,W93F), also known as mFAP10. An additional 14 designs (including mFAP_pH) were constructed by combining loop7 and hydrophobic $\beta$ barrel core mutations from the variants demonstrating the highest fluorescence intensity of the deprotonated state of DFHBI from the mFAP2.2 loop7 combinatorial library (Supplementary Data 4) and mFAP2.2 hydrophobic $\beta$-barrel core combinatorial library (Supplementary Data 5 and Supplementary Fig. 1g, h).

Design of chromophore-selective mFAPs. Computational modeling of DFHBI (Fig. 1b) into the binding pocket of mFAP2b (Fig. 1f) and mFAP2a (Fig. 1h) using Rosetta ${ }^{23,43}$ macromolecular modeling software showed that the mutations $\mathrm{V} 13 \mathrm{~A}$ and M15F resulted in a void in the binding pocket of mFAP2a. It was hypothesized that a commercially available structural derivative of the DFHBI chromophore with a trifluoromethyl group, DFHBI- $1 \mathrm{~T}^{4}$ (Fig. 1c), could pack into the void without causing steric clashes. Computational modeling of DFHBI-
$1 \mathrm{~T}$ in the pocket of mFAP2a (Fig. 1i) demonstrated good protein-chromophore shape complementarity, whereas DFHBI-1T modeled into the mFAP2b pocket (Fig. 1g) resulted in steric clashes. Studying the fluorescence of mFAP2a and mFAP2b in the presence of DFHBI-1T experimentally validated this (Fig. 1d, e). Relative fluorescence intensities and binding affinities of DFHBI, DFHBI-1T, and DFHO for selected mFAP variants were then measured at neutral pH (Supplementary Table 1). The fluorescence intensity of mFAP2a with DFHBI-1T was improved upon screening the "IN2" combinatorial library, resulting in mFAP10.

Design of extended loop library. $\beta$-hairpin loop fragments from the RCSB Protein Data Bank [www.rcsb.org] were used to manually curate custom $\beta$-barrel loop fragment databases. RosettaRemodel ${ }^{44}$ was used to fix the $\beta$-hairpin loop termini to loops $1,3,5$, and 7 of the de novo $\beta$-barrel scaffolds b11 and b32 $2^{1}$, picking 3-mer and 9-mer fragments from the custom $\beta$-barrel loop fragment databases, from which 2226 designs with successfully closed loops were generated. 
Table 3 Potential advantages of de novo designed mFAP sensors over existing fluorescent protein-based reporters and sensors.

\section{Biophysical property}

Size

Photostability

Reversible fluorescence

Ratiometric fluorescence

dynamic range

Low-Ca ${ }^{2+}$ affinity

Rapid response of split mFAPs

Reversibility

of split mFAPs

\section{Advantage}

Smaller size ${ }^{1}$ than GFP-based ${ }^{8}$, DiBs $^{15}$ and FAST-based 7,16 reporters (Supplementary Data 1).

Higher photostability over AcGFP1 in fixed mammalian cells (Fig. 2).

Spatiotemporal control over fluorescence via on-demand labeling protocols (Supplementary Fig. 4), unlike intrinsically fluorescent proteins ${ }^{8,50}$, and like $\mathrm{DiBs}^{15}$ and FAST-based ${ }^{7,16}$ reporters.

Higher ratiometric fluorescence dynamic range across the physiologically relevant $\mathrm{pH}$ range than pHRed20,51 and pHluorin2 21 (Fig. 3).

Lower $\mathrm{Ca}^{2+}$ affinity than existing fluorescent protein-based $\mathrm{Ca}^{2+}$ sensors such as $\mathrm{GCaMPer}^{34}$ and $\mathrm{CatchER}^{18}$ for imaging $\mathrm{Ca}^{2+}$ transients in high-Ca ${ }^{2+}$ concentration environments (Fig. $5 f$ and Supplementary Table 2). Rapid change in fluorescence intensity upon split mFAP fragment assembly (Fig. 6d), similar to splitFAST in not requiring chromophore maturation like split GFP10,11

Reversible fluorescence of split mFAPs, unlike split GFP10,11, and similar to splitFAST ${ }^{13}$ but with lower fragment affinities (Fig. $6 f$ and Supplementary Fig. 20).
Loop coordinates were extracted as .pdb files and used as templates to generate Rosetta blueprint files specifying amino acid type, secondary structure, and $\mathrm{ABEGO}^{45}$ type of loops to be rebuilt onto loops $1,3,5$, and 7 of a computational model of mFAP2b, resulting in 8904 Rosetta blueprint files. For each blueprint file, a RosettaScripts ${ }^{22,23}$ XML script (Supplementary Note 2) was used to graft the loop onto mFAP2b with a centroid energy function followed by Monte Carlo ${ }^{46}$ sampling of protein side-chain repacking and protein side-chain and backbone minimization steps in a full-atom Cartesian coordinate energy function ${ }^{47}$. Seven-thousand sevenhundred forty-eight resulting designs were filtered for the following computational protein design metrics (as scored from the XML script described in Supplementary Note 2): geometry $=1$; total_score_res $\leq-3.72714$; holes $\leq-1.2729$; pstat $\geq$ 0.755044; buns_sc heavy $\leq 2$; buns_bb heavy $\leq 2$; interfE $\leq-38.375$; $\mathrm{SC} \geq$ 0.734076 ; p_aa_pp $\leq-40.8947$; and omega $\leq 2.8757$. Only 59 designs with extended loops built onto loops 1, 3 and 7 of mFAP2b passed the filter criteria for experimental testing (Supplementary Data 11)

Design of $\mathrm{Ca}^{2+}$-responsive mFAPs. The mFAP2b loop7 sequence and the five extended loop7 sequences shown to confer fluorescence (Supplementary Fig. 6) were sampled as linkers for grafting the sequence of one EF-hand motif from Protein Data Bank (PDB) accession code $1 \mathrm{NKF}^{24}$ onto loop7 of mFAP2b. An inhouse script (Supplementary Note 3) was written to prune the experimentally validated extended loop7 sequences one residue at a time keeping up to four residues on the $\mathrm{N}$-terminal and C-terminal linkers relative to the grafted EF-hand motif, optionally adding an additional glycine residue on the $\mathrm{N}$-terminal linker and optionally adding an additional glycine or proline residue on the C-terminal linker. This combinatorial library (Supplementary Data 12) had a theoretical diversity of 1140 linkers. The linkers resulting in positively and negatively allosteric $\mathrm{Ca}^{2+}$. responsive mFAPs containing one EF-hand motif were combinatorially sampled to act as linkers for grafting two EF-hand motifs from PDB accession code $1 \mathrm{FW} 4^{25}$ onto loop7 of mFAP2b, where the N-terminal helix of PDB accession code 1FW4 was truncated up to homologous residues on successfully grafted single EF-hand motif designs. This combinatorial library (Supplementary Data 13) had a theoretical diversity of 385 linkers. The linkers resulting in negatively allosteric $\mathrm{Ca}^{2+}$. responsive mFAPs containing two EF-hand motifs were combinatorially sampled to act as linkers for grafting four EF-hand motifs from PDB accession code 1 PRW $^{26}$ onto loop7 of mFAP2b, where the N-terminal helix of PDB accession code 1PRW was truncated up to homologous residues on successfully grafted single EFhand motif designs. This combinatorial library (Supplementary Data 14) had a theoretical diversity of 25 linkers.

Design of split mFAPs and cpmFAPs. Split mFAPs were designed by manually inspecting the single-chain mFAP2a computational design model. In designing split mFAP fusions to BCL2 family heterodimers, linker compositions and lengths were chosen by manually inspecting the split mFAP2a computational design models and available crystal structures (PDB accession codes 5JSN and 5JSB). Split mFAP2a fragments were fused to maltose-binding protein, BCL2, aBCL2, BFL1, aBFL1, BCLXL, and aBCLXL after cysteine residues unlikely to be participating in disulfide bonds were mutated to serine or alanine residues (Supplementary Data 1).

Circularly permuted mFAP2a and mFAP2b were generated from mFAP2a and mFAP2b computational models using Rosetta ${ }^{23}$ and custom scripts in which Nand C-termini ("split points") were selected at mFAP loop2, loop4, loop6, and loop7 locations, and the two N-terminal and two C-terminal residues of cpmFAP scaffolds were re-designed compared to their respective residue types in MFAP2a (Supplementary Note 4). De novo structured and unstructured linkers covalently fusing the canonical mFAP termini were designed using RosettaScripts ${ }^{22,23}$, and 4000 resulting designs were filtered and sorted on design metrics. The top 12 designs were chosen for experimental testing after 3 circularly permuted mFAP2b variants were mutated to circularly permuted mFAP2a variants using the (V13A, $\mathrm{M} 15 \mathrm{~F}$ ) double point mutation (in canonical mFAP residue numbering)
(Supplementary Fig. 22a, b). In the subsequent round of cpmFAP designs, the de novo designed linker sequences from cp35-34_mFAP2a_12, cp35-34_mFAP2a_10, cp35-34_mFAP2a_08, and cp35-34_mFAP2a_11 were each sampled with the four split points described above, and the two $\mathrm{N}$-terminal and two $\mathrm{C}$-terminal residues in the cpmFAP were reverted back to their respective residue types in mFAP2a (Supplementary Fig. 22d and Supplementary Data 1).

Synthetic DNA construction. For combinatorial libraries, oligonucleotides with degenerate codons encoding desired mFAP sequences were designed using Swif$\mathrm{tLib}^{48}$. Overlapping forward and reverse oligonucleotides with degenerate and/or non-degenerate codons spanning the mFAP gene of interest were synthesized (IDT DNA). Oligonucleotides spanning identical gene regions were pooled at equimolar ratios relative to the theoretical amino acid diversity encoded by each gene region. Full-length genes were constructed using assembly polymerase chain reaction (PCR) with Phusion polymerase (NEB). For the extended loop library, the loop1, loop3, and loop7 libraries were assembled separately, and the concentrations of assembly PCR products consisting of full-length genes were quantified on a NanoDrop 8000 (Thermo Scientific) and the three libraries pooled in quantities proportional to the theoretical library diversities of assembly PCR products. Successfully assembled full-length genes, as well as synthetic gBlock (IDT DNA) oligonucleotides encoding full-length protein sequences, with $5^{\prime}$ and $3^{\prime}$ flanking vector backbone sequences were sub-cloned into the $\mathrm{pET} 15 \mathrm{~b}$ vector (Novagen) or the pcDNA5/FRT/TO vector (ThermoFisher Scientific) using Gibson assembly. The mFAP2.2 loop7 combinatorial library was constructed via Gibson assembly of polyacrylamide gel electrophoresis (PAGE)-purified duplex oligonucleotides into pET15b-mFAP2.2 linear vector DNA. The full-length genes encoding pHRed 20 and pHluorin $2^{21}$ were synthesized and cloned into the $\mathrm{pET} 29 \mathrm{~b}(+)$ vector (IDT DNA). Cloned DNA constructs were transformed into Lemo21(DE3) competent E. coli (NEB) and plated onto lysogeny broth (LB)-agar plates supplemented with $50.0 \mu \mathrm{g} \mathrm{mL}^{-1}$ carbenicillin or $50.0-100 \mu \mathrm{g} \mathrm{mL}^{-1}$ kanamycin.

Screening libraries. The number of $E$. coli colonies picked for functional screening was approximately 4 -fold the theoretical diversity of each library. E. coli colonies were inoculated into $1.00 \mathrm{~mL}$ of LB media supplemented with $50.0 \mu \mathrm{g} \mathrm{mL}^{-1}$ carbenicillin in Nunc $2.0 \mathrm{~mL}$ DeepWell 96-well plates (Thermo Scientific), and were grown at $37^{\circ} \mathrm{C}$ shaking at $1200 \mathrm{rpm}$ overnight. Twenty-five microliters of these cultures were inoculated into $1.00 \mathrm{~mL}$ of fresh LB media supplemented with $50.0 \mu \mathrm{g} \mathrm{mL} \mathrm{L}^{-1}$ carbenicillin, grown at $37^{\circ} \mathrm{C}$ and $1200 \mathrm{rpm}$ for $3-4 \mathrm{~h}$, then $0.5 \mathrm{mM}$ isopropyl $\beta$-D-thiogalactopyranoside (IPTG) final concentration was added to each well, and protein expression induced for $4 \mathrm{~h}$ at $37^{\circ} \mathrm{C}$ shaking at $1200 \mathrm{rpm}$. Cells were pelleted at $2272 \times g$ for $2-5 \mathrm{~min}$ and pellets were resuspended in $50.0 \mu \mathrm{L}$ of lysis buffer \#1 (25.0 mM Tris, $300 \mathrm{mM} \mathrm{NaCl}, 20.0 \mathrm{mM}$ imidazole, pH 8.00) supplemented with $1.00 \mathrm{mg} \mathrm{mL}^{-1} \mathrm{PMSF}$, a small amount of deoxyribonuclease I (DNase I) from bovine pancreas (Sigma Aldrich), and $2.00 \mathrm{mg} \mathrm{mL}^{-1}$ lysozyme from chicken egg white (Sigma) for lysis. Crude lysates were vigorously shaken at $25-37^{\circ} \mathrm{C}$ for $12-48 \mathrm{~h}$, then clarified by centrifugation. Clarified lysates were assayed on a Synergy Neo2 hybrid multi-mode reader (BioTek) in 96-well non-binding surface microplates (Corning 3650) with Gen5 (version 3.03.14) software.

For each clone encoding a $\beta$-barrel core variant, loop7 variant, or extended loop variant, $15.0 \mu \mathrm{L}$ of clarified lysate was combined with $185 \mu \mathrm{L}$ of $\mathrm{Na}_{2} \mathrm{HPO}_{4}$-citrate (pH 7.36 or pH 3.66) buffer supplemented with $150 \mathrm{mM} \mathrm{NaCl}$ and either $1.08 \mu \mathrm{M}$ DFHBI (Lucerna), $555 \mathrm{nM}$ DFHBI (Lucerna), or $250 \mathrm{nM}$ DFHBI-1T (Lucerna). $\mathrm{Na}_{2} \mathrm{HPO}_{4}$-citrate buffer was made from $200 \mathrm{mM} \mathrm{Na} \mathrm{HPO}_{4}$ and $100 \mathrm{mM}$ citrate stock solutions, and final $\mathrm{pH}$ was adjusted using hydrochloric acid $(\mathrm{HCl})$ or sodium hydroxide $(\mathrm{NaOH})$. DFHBI and DFHBI-1T stock solutions were $2.00 \mathrm{mM}$ in $23.8 \mathrm{mM}$ Tris ( $\mathrm{pH} 8.00$ ), $95.0 \mathrm{mM} \mathrm{NaCl}$, and $5 \%$ dimethyl sulfoxide (DMSO). Clones that demonstrated fluorescence were Sanger sequenced via colony PCR of overnight cultures, and the brightest designs or designs with highest fluorescence 
fold-change across $\mathrm{pH}$ 7.36-3.66 were further characterized with large-scale protein purification ${ }^{49}$.

For each clone encoding one or more EF-hand motifs grafted onto $\beta$-barrel loop7, $15.0 \mu \mathrm{L}$ of lysate was combined with $185 \mu \mathrm{L}$ of either $2.00 \mathrm{mM} \mathrm{CaCl}_{2}$ (Sigma Aldrich), $25.0 \mathrm{mM}$ Tris (pH 8.00), $100 \mathrm{mM} \mathrm{NaCl}, 1.08 \mu \mathrm{M}$ DFHBI or $2.00 \mathrm{mM}$ EGTA (Sigma Aldrich), $25.0 \mathrm{mM}$ Tris (pH 8.00), $100 \mathrm{mM} \mathrm{NaCl}, 1.08 \mu \mathrm{M}$ DFHBI. Clones that demonstrated greater than an approximately twofold change in fluorescence intensity between $\mathrm{CaCl}_{2}$ and EGTA conditions were Sanger sequenced, and the designs demonstrating the highest fold-change in fluorescence intensity between $\mathrm{CaCl}_{2}$ and EGTA conditions were further characterized with large-scale protein purification ${ }^{49}$.

Fluorescence intensity assays. To measure fluorescence intensities of the phenolate form of chromophores, fluorescence was measured on a Synergy Neo2 hybrid multi-mode reader (BioTek) in flat bottom, black polystyrene, non-binding surface 96-well microplates (Corning 3650). Fluorescence intensity was measured in triplicate by exciting at $\lambda_{\mathrm{ex}}=484 \mathrm{~nm}$ and measuring fluorescence emission at $\lambda_{\mathrm{em}}=505 \mathrm{~nm}$ (or $\lambda_{\mathrm{em}}=511 \mathrm{~nm}$ for clones harboring the W27 mutation) (Supplementary Fig. 1g, h). The W27 mutation redshifts the peak emission wavelength from $\lambda_{\mathrm{em}}=\sim 505 \mathrm{~nm}$ to $\lambda_{\mathrm{em}}=\sim 511 \mathrm{~nm}$, presumably due to the W27 indole ring donating a hydrogen bond to the imidazolinone moiety of the deprotonated state of DFHBI, which stabilizes the chromophore conjugated $\pi$-electron system in the excited state causing the redshift in emission ${ }^{50}$. Fifteen microliters of small-scale purified protein ${ }^{49}$ was combined with $185 \mu \mathrm{L}$ of $\mathrm{Na}_{2} \mathrm{HPO}_{4}$-citrate (pH 7.36) buffer supplemented with $150 \mathrm{mM} \mathrm{NaCl}$ and $108 \mathrm{nM}$ DFHBI for a final concentration of $100 \mathrm{nM}$ DFHBI. In measuring the excitation spectra from $\lambda_{\mathrm{em}}=525 \mathrm{~nm}$ of each clone in triplicate (Supplementary Fig. 1i), $30.0 \mu \mathrm{L}$ of large-scale purified protein ${ }^{49}$ was combined with $170 \mu \mathrm{L}$ of $25.0 \mathrm{mM}$ Tris ( $\mathrm{pH} 8.00$ ) supplemented with $100 \mathrm{mM}$ $\mathrm{NaCl}$ and $914 \mathrm{nM}$ DFHBI for a final concentration of $7.77 \mu \mathrm{M}$ protein and $777 \mathrm{nM}$ DFHBI. In measuring the fluorescence intensity at $\lambda_{\mathrm{ex}}=484 \mathrm{~nm}$ and $\lambda_{\mathrm{em}}=509 \mathrm{~nm}$ of each clone in triplicate (Supplementary Fig. 1j), $30.0 \mu \mathrm{L}$ of large-scale purified protein ${ }^{49}$ was combined with $170 \mu \mathrm{L}$ of $25.0 \mathrm{mM}$ Tris ( $\mathrm{pH} 8.00$ ) supplemented with $100 \mathrm{mM} \mathrm{NaCl}$ and $653 \mathrm{nM}$ DFHBI for a final concentration of $5.55 \mu \mathrm{M}$ protein and $555 \mathrm{nM}$ DFHBI. In measuring fluorescence intensity at $\lambda_{\mathrm{ex}}=468 \mathrm{~nm}$ and $\lambda_{\mathrm{em}}=$ $530 \mathrm{~nm}$ of each clone in technical triplicate (Supplementary Fig. $1 \mathrm{k}$ ), $24.0 \mu \mathrm{L}$ of $35.4 \mu \mathrm{M}$ large-scale purified protein ${ }^{49}$ was combined with $1.00 \mu \mathrm{L}$ of $1.25 \mu \mathrm{M}$ DFHBI or $1.00 \mu \mathrm{L}$ of $1.25 \mu \mathrm{M}$ DFHBI-1T (Lucerna) (from $2 \mathrm{mM}$ chromophore stock solutions dissolved in $0.5 \%$ DMSO and $99.5 \%$ high-salt Tev cleavage buffer [25.0 mM Tris, $100 \mathrm{mM} \mathrm{NaCl}, \mathrm{pH}$ 8.00]) for final concentrations of $34.0 \mu \mathrm{M}$ protein and $50.0 \mathrm{nM}$ chromophore. The mFAP9-DFHBI complex had $\sim 1.1$-fold the fluorescence intensity of the mFAP10-DFHBI complex, and the mFAP9-DFHBI-1T complex had $\sim 0.75$-fold the fluorescence intensity of the mFAP10-DFHBI-1T complex (Supplementary Fig. 1k). In collecting fluorescence emission spectra by exciting fluorescence at $\lambda_{\mathrm{ex}}=484 \mathrm{~nm}$ and collecting fluorescence emission at $\lambda_{\mathrm{em}}=495-650 \mathrm{~nm}$ (Supplementary Fig. 6b), and in measuring fluorescence endpoints in techincal triplicate at unique peak excitation and emission wavelengths (Supplementary Fig. 6c), $195 \mu \mathrm{L}$ of large-scale purified protein ${ }^{49}$ was combined with $5.00 \mu \mathrm{L}$ of $25 \mathrm{mM}$ Tris (pH 8.00) supplemented with $100 \mathrm{mM}$ $\mathrm{NaCl}$ and $40.0 \mu \mathrm{M}$ DFHBI for a final concentration of $10.0 \mu \mathrm{M}$ protein and 1.00 $\mu \mathrm{M}$ DFHBI.

In measuring fluorescence intensity of small-scale purified proteins ${ }^{49}$ (Supplementary Table 1), wells were excited at $\lambda_{\mathrm{ex}}=488 \mathrm{~nm}$ for DFHBI and DFHBI-1T and $\lambda_{\mathrm{ex}}=505 \mathrm{~nm}$ for DFHO, and fluorescence emission measured at $\lambda_{\mathrm{em}}=510 \mathrm{~nm}$ for DFHBI and DFHBI-1T and $\lambda_{\mathrm{em}}=545 \mathrm{~nm}$ for DFHO. Fluorescence endpoints were measured using either $25.0 \mu \mathrm{L}$ or $50.0 \mu \mathrm{L}$ of smallscale purified protein in either $175 \mu \mathrm{L}$ or $150 \mu \mathrm{L}$, respectively, of $\mathrm{Na}_{2} \mathrm{HPO}_{4}$-citrate (pH 7.36) buffer supplemented with $150 \mathrm{mM} \mathrm{NaCl}$ and either $100 \mathrm{nM}$ or $10.0 \mu \mathrm{M}$ of either DFHBI (Lucerna), DFHBI-1T (Lucerna) or DFHO (Lucerna) (from 20.0 $\mathrm{mM}$ chromophore stock solutions in $100 \%$ DMSO), for $200 \mu \mathrm{L}$ final volumes per well.

To measure the fluorescence intensities of circularly permuted mFAP2a variants (Supplementary Fig. 22b, d), fluorescence endpoints were measured on a Synergy $\mathrm{Neo} 2$ hybrid multi-mode reader (BioTek) in flat bottom, black polystyrene, nonbinding surface 96-well microplates (Corning 3650) or half-area microplates (Corning 3686). Fluorescence endpoints were measured in technical triplicate by exciting at $\lambda_{\mathrm{ex}}=488 \mathrm{~nm}$ and measuring fluorescence emission at $\lambda_{\mathrm{em}}=510 \mathrm{~nm}$ (Supplementary Fig. 22b), or exciting at $\lambda_{\mathrm{ex}}=468 \mathrm{~nm}$ and measuring fluorescence emission at $\lambda_{\mathrm{em}}=530 \mathrm{~nm}$ (Supplementary Fig. 22d). Ninety microliters of $55.6 \mu \mathrm{M}$ large-scale purified protein ${ }^{49}$ in high-salt Tev cleavage buffer was combined with $10.0 \mu \mathrm{L}$ of $5.00 \mu \mathrm{M}$ DFHBI-1T in high-salt Tev cleavage buffer for final concentrations of $50.0 \mu \mathrm{M}$ protein and $500 \mathrm{nM}$ DFHBI-1T in $100 \mu \mathrm{L}$ final volumes (Supplementary Fig. 22b), or $48.0 \mu \mathrm{L}$ of $41.7 \mu \mathrm{M}$ large-scale purified protein ${ }^{49}$ in high-salt Tev cleavage buffer was combined with $2.00 \mu \mathrm{L}$ of $1.25 \mu \mathrm{M}$ DFHBI-1T in high-salt Tev cleavage buffer for final concentrations of $40.0 \mu \mathrm{M}$ protein and $50.0 \mathrm{nM}$ DFHBI-1T in $50.0 \mu \mathrm{L}$ final volumes (Supplementary Fig. 22d).

Densitometry. In quantifying relative protein expression levels in E. coli (Supplementary Table 1), $5.00 \mu \mathrm{L}$ of small-scale purified proteins ${ }^{49}$ were combined with $5.00 \mu \mathrm{L}$ of $2 \mathrm{x}$ Laemmli buffer, denatured at $99^{\circ} \mathrm{C}$ for $10 \mathrm{~min}$, and $5.00 \mu \mathrm{L}$ of each sample run on Any kD Mini-PROTEAN TGX Stain-Free Precast Gels (Bio-Rad) in
Tris-glycine buffer alongside $5.00 \mu \mathrm{L}$ of Precision Plus Protein Unstained Protein Standard (Bio-Rad). Gels were stained using an eStain L1 Protein Staining System (GenScript) and imaged using a Molecular Imager ChemiDoc XRS+ (Bio-Rad). Relative densitometry was analyzed in Image Lab Software Version 6.0.1 build 34 Standard Edition (Bio-Rad) referenced to the $15 \mathrm{kDa}$ protein ladder band.

pH-dependent fluorescence assays. pH-dependent fluorescence was measured on a Synergy Neo2 hybrid multi-mode reader in flat bottom, black polystyrene, non-binding surface 96-well microplates (Corning 3650). In measuring the $\mathrm{pH}-$ sensitivity of $\beta$-barrel variants in technical triplicate (Supplementary Fig. 5a, b), $20.0 \mu \mathrm{L}$ of large-scale purified protein ${ }^{49}$ was combined with $180 \mu \mathrm{L}$ of $\mathrm{Na}_{2} \mathrm{HPO}_{4-}$ citrate (pH 7.34 or 3.61 ) supplemented with $150 \mathrm{mM} \mathrm{NaCl}$ and $278 \mathrm{nM}$ DFHBI for final concentrations of $2.50 \mu \mathrm{M}$ protein and $250 \mathrm{nM}$ DFHBI. Buffer wells for background subtraction were prepared identically except using $20.0 \mu \mathrm{L}$ of high-salt Tev cleavage buffer instead of purified protein. Wells were excited at $\lambda_{\text {ex }}=387 \mathrm{~nm}$ or $\lambda_{\mathrm{ex}}=484 \mathrm{~nm}$ and fluorescence emission measured at $\lambda_{\mathrm{em}}=501 \mathrm{~nm}$ or $\lambda_{\mathrm{em}}=$ $505 \mathrm{~nm}$, respectively. Following background subtraction from the mean endpoint fluorescence of buffer controls, the fluorescence ratio fold-change from $\mathrm{pH}$ 3.61-7.34 was calculated as:

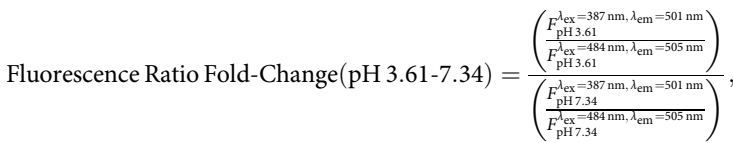

where $F_{\mathrm{pH}}^{\lambda_{\mathrm{ex}}, \lambda_{\mathrm{em}}}$ is the endpoint fluorescence measurement at the subscripted $\mathrm{pH}$ value and superscripted fluorescence excitation $\left(\lambda_{\mathrm{ex}}\right)$ and fluorescence emission $\left(\lambda_{\mathrm{em}}\right)$ wavelengths.

In measuring $\mathrm{pH}$-sensitivity of $\beta$-barrel variants (Supplementary Fig. 1f) in technical triplicate, $140 \mu \mathrm{L}$ of $\mathrm{Na}_{2} \mathrm{HPO}_{4}$-citrate ( $\mathrm{pH} 7.36$ or 3.66) supplemented with $150 \mathrm{mM} \mathrm{NaCl}$ and $1.43 \mu \mathrm{M}$ DFHBI was combined with $10.0 \mu \mathrm{L}$ of small-scale purified protein ${ }^{49}$ for a final concentration of $1.33 \mu \mathrm{M}$ DFHBI. Fluorescence endpoints were measured by exciting at $\lambda_{\mathrm{ex}}=387 \mathrm{~nm}$ or $\lambda_{\mathrm{ex}}=483 \mathrm{~nm}$ and measuring fluorescence emission at $\lambda_{\mathrm{em}}=501 \mathrm{~nm}$ or $\lambda_{\mathrm{em}}=504 \mathrm{~nm}$, respectively. Without background subtraction, the fluorescence ratio fold-change from $\mathrm{pH}$ 3.66-7.36 was calculated as:

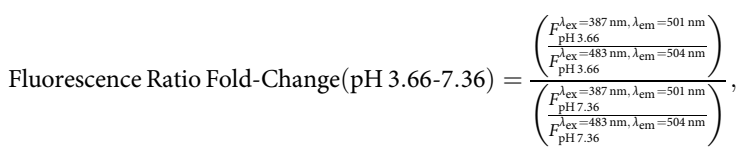

where $F_{\mathrm{pH}}^{\lambda_{\mathrm{ex}}, \lambda_{\mathrm{em}}}$ is the mean of three technical replicates of endpoint fluorescence measurements at the subscripted $\mathrm{pH}$ value and superscripted fluorescence excitation $\left(\lambda_{\mathrm{ex}}\right)$ and fluorescence emission $\left(\lambda_{\mathrm{em}}\right)$ wavelengths.

In measuring $\mathrm{pH}$-dependent fluorescence (Fig. $3 \mathrm{~d}-\mathrm{i}$ ), $\mathrm{Na}_{2} \mathrm{HPO}_{4}$-citrate buffer supplemented with $150 \mathrm{mM} \mathrm{NaCl}$ at unique $\mathrm{pH}$ values were produced via mixing various volumes of $100 \mathrm{mM}$ citric acid (Sigma Aldrich), $200 \mathrm{mM} \mathrm{Na}_{2} \mathrm{HPO}_{4}$ (Sigma Aldrich), and $2.00 \mathrm{M} \mathrm{NaCl}$, and final pHs quantified via an Accumet AB15 Basic $\mathrm{pH}$ meter (Fisher Scientific). pHRed and pHluorin2 were produced via large-scale protein purification, 6xHis-tag removal and size-exclusion chromatography (SEC) purification, and mFAP_pH and mFAP2b were produced via large-scale protein purification and SEC purification ${ }^{49}$. $\mathrm{pH}$-dependent fluorescence was measured at $500 \mathrm{nM}$ final protein concentration for mFAP2b, mFAP_pH and pHRed, and $170 \mathrm{nM}$ final concentration for pHluorin2, at each $\mathrm{pH}$ in technical triplicate in $200 \mu \mathrm{L}$ final volumes per well. To prevent $\mathrm{pH}$ fluctuations upon addition of protein and DFHBI, $195 \mu \mathrm{L}$ of $\mathrm{Na}_{2} \mathrm{HPO}_{4}$-citrate buffers supplemented with $150 \mathrm{mM} \mathrm{NaCl}$ at each $\mathrm{pH}$ was aliquoted per well, $4.00 \mu \mathrm{L}$ of purified protein was aliquoted per well, and $1.00 \mu \mathrm{L}$ of $1.00 \mathrm{mM}$ DFHBI (in 2.5\% DMSO and $97.5 \%$ high-salt Tev cleavage buffer) was added to wells containing mFAP_pH, whereas $1.00 \mu \mathrm{L}$ of chromophore buffer (2.5\% DMSO and $97.5 \%$ high-salt Tev cleavage buffer) was added to wells containing pHRed or pHluorin2. Blank wells for background subtraction for mFAP2b, mFAP_pH, pHRed and pHluorin2 were prepared identically, respectively, except adding $4.00 \mu \mathrm{L}$ of high-salt Tev cleavage buffer instead of purified protein. In measuring fluorescence excitation spectra at each $\mathrm{pH}$ (Fig. 3d), excitation wavelengths were set to the range $\lambda_{\mathrm{ex}}=300-530 \mathrm{~nm}$ and fluorescence emission measured at $\lambda_{\mathrm{em}}=562 \mathrm{~nm}$. In measuring $\mathrm{pH}$-dependent fluorescence excitation spectra at $\mathrm{pH} 3.76$ and $\mathrm{pH} 7.34$ (Fig. 3f, g), excitation spectra were measured using excitation wavelengths in the range $\lambda_{\mathrm{ex}}=300-540 \mathrm{~nm}$ and emission wavelength $\lambda_{\mathrm{em}}=592 \mathrm{~nm}$.

In measuring fluorescence emission spectra at two $\mathrm{pH}$ values (Fig. 3e), emission spectra for the blueshifted excitation peak was measured at $\mathrm{pH} 3.63$ using excitation wavelength $\lambda_{\mathrm{ex}}=379 \mathrm{~nm}$ and emission wavelengths in the range $\lambda_{\mathrm{em}}=$ $460-700 \mathrm{~nm}$, and emission spectra for the redshifted excitation peak was measured at $\mathrm{pH} 8.38$ using excitation wavelength $\lambda_{\mathrm{ex}}=430 \mathrm{~nm}$ and emission wavelengths in the range $\lambda_{\mathrm{em}}=460-700 \mathrm{~nm}$. In measuring fluorescence from both the blueshifted and redshifted excitation peaks (Fig. $3 \mathrm{~h}$, i), for mFAP_pH the fluorescence excitation wavelengths were $\lambda_{\mathrm{ex}}=379 \mathrm{~nm}$ and $\lambda_{\mathrm{ex}}=483 \mathrm{~nm}$ and fluorescence emission wavelengths were $\lambda_{\mathrm{em}}=498 \mathrm{~nm}$ and $\lambda_{\mathrm{em}}=503 \mathrm{~nm}$, respectively, whereas for pHRed the fluorescence excitation wavelengths were $\lambda_{\mathrm{ex}}=440 \mathrm{~nm}$ and $\lambda_{\mathrm{ex}}=$ $575 \mathrm{~nm}$ and fluorescence emission wavelengths were both $\lambda_{\mathrm{em}}=635 \mathrm{~nm}$, and for 
pHluorin2 the fluorescence excitation wavelengths were $\lambda_{\mathrm{ex}}=405 \mathrm{~nm}$ and $\lambda_{\mathrm{ex}}=$ $485 \mathrm{~nm}$ and fluorescence emission wavelengths were both $\lambda_{\mathrm{em}}=535 \mathrm{~nm}$. In Fig. $3 \mathrm{i}$, for the mFAP_pH-DFHBI complex, pHRed, and pHluorin2 the ratiometric fluorescence $\left(F_{\text {ratio }}\right)$ is calculated from the background-subtracted, unnormalized fluorescence measurements using fluorescence emission from the redshifted excitation peak ( $\left.F^{\text {redshifted } \lambda_{\mathrm{ex}}}\right)$ as the numerator and fluorescence emission from the blueshifted excitation peak ( $F^{\text {blueshifted } \lambda_{\mathrm{ex}}}$ ) as the denominator:

$$
F_{\text {ratio }}=\frac{F^{\text {redshifted } \lambda_{\text {ex }}}}{F^{\text {blueshifted } \lambda_{\text {ex }}}}
$$

In Fig. 3i, mean ratiometric fluorescence values are fit to continuous logistic

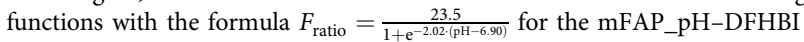
complex, $F_{\text {ratio }}=\frac{4.20}{1+\mathrm{e}^{2.00(-1 \mathrm{PH}-6.60)}}$ for $\mathrm{pHRed}$, and $F_{\text {ratio }}=\frac{5.96}{1+\mathrm{e}^{0.522 .(\mathrm{pH}-2.20)}}$ for pHluorin2 using non-linear least squares fitting, which serve as continuous calibration curves for real-time quantification of $\mathrm{pH}^{51}$.

In measuring the protonated (phenolic) and deprotonated (phenolate) DFHBI affinities with mFAP_pH (Supplementary Fig. 5c), fluorescence endpoints were measured on a Synergy Neo2 hybrid multi-mode reader (BioTek) in flat bottom, black polystyrene, non-binding surface 96-well microplates (Corning 3650). mFAP_pH was produced by large-scale protein purification ${ }^{49}$ and aliquoted to $200 \mu \mathrm{L}$ final volumes at $500 \mathrm{nM}$ final concentration in seven serial dilutions $(\sqrt{10}$ dilution factor) of DFHBI starting from $10.0 \mu \mathrm{M}$ DFHBI final concentration, including an eighth condition without chromophore, in $\mathrm{Na}_{2} \mathrm{HPO}_{4}$-citrate buffer supplemented with $143 \mathrm{mM} \mathrm{NaCl}$ final concentration at either $\mathrm{pH} 3.61$ or $\mathrm{pH} 7.34$. For $\mathrm{pH} 3.61$ fluorescence was excited at $\lambda_{\mathrm{ex}}=379 \mathrm{~nm}$ and fluorescence emission measured at $\lambda_{\mathrm{em}}=498 \mathrm{~nm}$, and for $\mathrm{pH} 7.34$ fluorescence was excited at $\lambda_{\mathrm{ex}}=$ $483 \mathrm{~nm}$ and fluorescence emission measured at $\lambda_{\mathrm{em}}=503 \mathrm{~nm}$. At each $\mathrm{pH}$ background fluorescence endpoints of wells with identical chromophore concentrations but purified protein replaced with an identical volume of buffer were measured, and fluorescence endpoints subtracted from those measured with protein. Background-subtracted data were normalized from 0 to 1 and fit to a single-binding site isotherm function using non-linear least squares fitting to obtain the reported $K_{\mathrm{d}}$ values, which were less than the protein concentrations tested.

Chromophore titrations. Fluorescence endpoints were measured on a Synergy Neo2 hybrid multi-mode reader (BioTek) in flat bottom, black polystyrene, nonbinding surface 96-well microplates (Corning 3650). In measuring chromophorebinding affinities (Fig. 1d, e), mFAP2, mFAP2a, and mFAP2b, and mFAP10 were produced by large-scale protein purification and SEC purification ${ }^{49}$. Proteins were aliquoted in eight technical replicates in $200 \mu \mathrm{L}$ final volumes to $20.0 \mathrm{nM}$ final concentration in ten serial dilutions ( $\sqrt{10}$ dilution factor) of DFHBI starting from $31.6 \mu \mathrm{M}$ DFHBI or $31.6 \mu \mathrm{M}$ DFHBI-1T final concentrations, including an eleventh condition without chromophore. Fluorescence was excited at $\lambda_{\mathrm{ex}}=468 \mathrm{~nm}$ and fluorescence emission measured at $\lambda_{\mathrm{em}}=530 \mathrm{~nm}$. Background fluorescence endpoints of wells with identical chromophore concentrations but purified protein replaced with an identical volume of high-salt Tev cleavage buffer were measured, and fluorescence endpoints subtracted from those measured with protein. Background-subtracted data were averaged and the means normalized from 0 to 1 and fit to a single-binding site isotherm function using non-linear least squares fitting to obtain a fitted $K_{\mathrm{d}}$ value (Table 1 ), and the fit scaled to the maximum mean value (Fig. 1d, e).

Chromophore affinities (Supplementary Table 1) were obtained by producing proteins via large-scale protein purification (some variants further purified by SEC) ${ }^{49}$ and preparing proteins at $25.0 \mu \mathrm{L}$ final volumes in flat bottom, black polystyrene, non-binding surface 96-well half-area microplates (Corning 3686). Proteins were aliquoted to $500 \mathrm{nM}$ final concentrations in high-salt Tev cleavage buffer (mFAP2.2.5 was prepared at $386 \mathrm{nM}$, and mFAP2a and mFAP3 were prepared at $50.0 \mathrm{nM}$ ) in eleven serial dilutions ( $\sqrt{10}$ dilution factor) from $1.00 \mathrm{mM}$ DFHBI, $1.00 \mathrm{mM}$ DFHBI-1T, or $1.00 \mathrm{mM}$ DFHO, including a twelfth condition without chromophore. Fluorescence endpoints were measured on a Synergy Neo2 hybrid multi-mode reader (BioTek) with excitation and emission wavelengths set differently between protein variants to achieve maximal fluorescence intensity. Background fluorescence endpoints of wells with identical chromophore concentrations but purified protein replaced with an identical volume of high-salt Tev cleavage buffer were measured, and fluorescence endpoints measured with identical instrument settings subtracted from those measured with protein prior to normalization

$\mathrm{Ca}^{2+}$-responsive mFAP DFHBI titrations. In measuring $\mathrm{Ca}^{2+}$-dependent DFHBI affinity of $\mathrm{Ca}^{2+}$-responsive mFAPs on a Synergy Neo2 hybrid multi-mode reader (Fig. 4a-c and Supplementary Figs. 10a, b, d, e, g, h, 11a, b, d, e, g, h, 12a, b, d, e, g, $\mathrm{h}, 16 \mathrm{a})$, large-scale purified proteins ${ }^{49}$ were aliquoted to a final concentration of $500 \mathrm{nM}$ in either $450 \mathrm{mM} \mathrm{CaCl}_{2}$ (Sigma Aldrich) (prepared in high-salt Tev cleavage buffer) or high-salt Tev cleavage buffer, along with ten serial dilutions ( $\sqrt{10}$ dilution factor) of DFHBI starting from $316 \mu \mathrm{M}$ DFHBI including an eleventh condition without chromophore. Final volumes were $25.0 \mu \mathrm{L}$ in flat bottom, black polystyrene, non-binding surface 96 -well half-area microplates (Corning 3686) or $200 \mu \mathrm{L}$ in flat bottom, black polystyrene, non-binding surface 96-well microplates (Corning 3650). Fluorescence endpoints were measured using excitation wavelength $\lambda_{\mathrm{ex}}=488 \mathrm{~nm}$ and emission wavelength $\lambda_{\mathrm{em}}=510 \mathrm{~nm}$. Background fluorescence endpoints of wells with identical chromophore concentrations lacking protein (substituted for equivalent volumes of high-salt Tev cleavage buffer) were measured and subtracted from protein measurements prior to normalization.

For DFHBI titrations of EF4n_mFAP2a and EF4n_mFAP2b (Supplementary Fig. 13a, b), a small amount of Chelex 100 sodium form (Sigma Aldrich) was added to purified protein (produced by large-scale protein purification and SEC purification ${ }^{49}$ ) and nutated at $4{ }^{\circ} \mathrm{C}$ overnight. High-salt Tev cleavage buffer with a small amount of Chelex 100 was prepared and mixed at room temperature overnight, and a $2.00 \mathrm{mM}$ DFHBI stock solution (in 5\% DMSO and 95\% high-salt Tev cleavage buffer) with a small amount of Chelex 100 was prepared and nutated overnight at $4{ }^{\circ} \mathrm{C}$. Proteins were aliquoted to a final concentration of $5.00 \mu \mathrm{M}$ in either $500 \mu \mathrm{M} \mathrm{CaCl}_{2}$ (Sigma Aldrich) (prepared in high-salt Tev cleavage buffer) or $500 \mu \mathrm{M}$ EGTA (Sigma Aldrich) (prepared in high-salt Tev cleavage buffer), along with ten serial dilutions ( $\sqrt{10}$ dilution factor) of DFHBI starting from $316 \mu \mathrm{M}$ DFHBI (using Chelex 100 pre-treated DFHBI stock solution and high-salt Tev cleavage buffer), including an eleventh condition without chromophore. Final volumes were $25.0 \mu \mathrm{L}$ in flat bottom, black polystyrene, non-binding surface 96well half-area microplates (Corning 3686). Fluorescence endpoints were measured using excitation wavelength $\lambda_{\mathrm{ex}}=488 \mathrm{~nm}$ and emission wavelength $\lambda_{\mathrm{em}}=510 \mathrm{~nm}$ for EF4n_mFAP2b, and excitation wavelength $\lambda_{\mathrm{ex}}=478 \mathrm{~nm}$ and emission wavelength $\lambda_{\mathrm{em}}=520 \mathrm{~nm}$ for EF4n_mFAP2a. Background fluorescence endpoints of wells with identical chromophore concentrations lacking protein (substituted for equivalent volumes of Chelex 100 pre-treated high-salt Tev cleavage buffer) were measured and subtracted from protein measurements prior to normalization.

$\mathrm{Ca}^{2+}$-responsive mFAP Ca' ${ }^{2+}$ titrations. In measuring $\mathrm{Ca}^{2+}$ affinity of mFAPs on a Synergy Neo2 hybrid multi-mode reader (Fig. $4 \mathrm{~d}, \mathrm{e}, \mathrm{f}$ and Supplementary Figs. 10c, f, i, 11c, f, i, 13c, f, i, 16b), large-scale purified proteins ${ }^{49}$ were aliquoted in technical triplicate to a final concentration of $500 \mathrm{nM}$ with $5.00 \mu \mathrm{M}$ DFHBI in eleven serial dilutions ( $\sqrt{10}$ dilution factor) of $\mathrm{CaCl}_{2}$ (Sigma Aldrich) starting from either $900 \mathrm{mM}$ or $90.0 \mathrm{mM} \mathrm{CaCl} 2$ (diluted using high-salt Tev cleavage buffer) including a twelfth condition without $\mathrm{CaCl}_{2}$. Final volumes were $200 \mu \mathrm{L}$ in flat bottom, black polystyrene, non-binding surface 96 -well microplates (Corning 3650). Fluorescence endpoints were measured using excitation wavelength $\lambda_{\mathrm{ex}}=$ $484 \mathrm{~nm}$ and emission wavelength $\lambda_{\mathrm{em}}=508 \mathrm{~nm}$. Background fluorescence endpoints of wells with identical chromophore and $\mathrm{CaCl}_{2}$ concentrations lacking protein (substituted for equivalent volumes of high-salt Tev cleavage buffer) were measured in technical triplicate, averaged per condition, and subtracted from protein measurement averages of the same conditions.

For $\mathrm{Ca}^{2+}$ titrations of EF4n_mFAP2a and EF4n_mFAP2b (Supplementary Fig. 13c), a small amount of Chelex 100 sodium form (Sigma Aldrich) was added to purified protein (produced by large-scale protein purification and SEC purification ${ }^{49}$ ) and nutated at $4^{\circ} \mathrm{C}$ overnight. EF4n_mFAP2a was aliquoted in technical triplicate to a final concentration of $8.00 \mu \mathrm{M}$ with $80.0 \mu \mathrm{M}$ DFHBI and EF4n_mFAP2b was aliquoted in technical triplicate to a final concentration of $6.25 \mu \mathrm{M}$ with $43.4 \mu \mathrm{M}$ DFHBI, in eleven serial dilutions ( $\sqrt{10}$ dilution factor) of $\mathrm{CaCl}_{2}$ starting from $9.00 \mathrm{mM}$ or $4.00 \mathrm{mM} \mathrm{CaCl}_{2}$ (diluted using high-salt Tev cleavage buffer pre-treated with Chelex 100) including a twelfth condition without $\mathrm{CaCl}_{2}$. Final volumes were $25.0 \mu \mathrm{L}$ in flat bottom, black polystyrene, non-binding surface 96-well half-area microplates (Corning 3686) or $200 \mu \mathrm{L}$ in flat bottom, black polystyrene, non-binding surface 96-well microplates (Corning 3650). Fluorescence endpoints were measured for EF4n_mFAP2a using excitation wavelength $\lambda_{\mathrm{ex}}=478 \mathrm{~nm}$ and emission wavelength $\lambda_{\mathrm{em}}=520 \mathrm{~nm}$, and for EF4n_mFAP2b using excitation wavelength $\lambda_{\mathrm{ex}}=484 \mathrm{~nm}$ and emission wavelength $\lambda_{\mathrm{em}}=508 \mathrm{~nm}$. Background fluorescence endpoints of wells with identical chromophore and $\mathrm{CaCl}_{2}$ concentrations lacking protein (substituted for equivalent volumes of high-salt Tev cleavage buffer) were measured in technical triplicate, averaged per condition, and subtracted from protein measurement averages of the same conditions. For non-linear least squares fitting to obtain $K_{\mathrm{d}}$ values, means were fit to a single-binding site isotherm function where $\mathrm{Ca}^{2+}$-binding sites were modeled independently with a Hill coefficient of 1 .

\section{$\mathrm{Ca}^{2+}$-responsive mFAP fluorescence spectra. Fluorescence spectra of $\mathrm{Ca}^{2+}$ -} responsive mFAPs were measured on a Synergy Neo2 hybrid multi-mode reader in flat bottom, black polystyrene, non-binding surface 96 -well microplates (Corning 3650). In Supplementary Fig. 9a-n, fluorescence excitation spectra were measured using excitation wavelengths $\lambda_{\mathrm{ex}}=350-530 \mathrm{~nm}$ and emission wavelength $\lambda_{\mathrm{em}}=$ $570 \mathrm{~nm}$, and fluorescence emission spectra measured using excitation wavelength $\lambda_{\mathrm{ex}}=430 \mathrm{~nm}$ and emission wavelengths $\lambda_{\mathrm{em}}=470-750 \mathrm{~nm}$. In Supplementary Fig. 9o-t, for optimized signal the fluorescence excitation spectra were measured using excitation wavelengths $\lambda_{\mathrm{ex}}=350-510 \mathrm{~nm}$ and emission wavelength $\lambda_{\mathrm{em}}=$ $550 \mathrm{~nm}$, and fluorescence emission spectra measured using excitation wavelength $\lambda_{\mathrm{ex}}=450 \mathrm{~nm}$ and emission wavelengths $\lambda_{\mathrm{em}}=490-700 \mathrm{~nm}$. 6xHis-tagged proteins were produced via large-scale protein purification ${ }^{49}$ and aliquoted to final volumes of $200 \mu \mathrm{L}$ per well with final concentrations of either $1.50 \mathrm{mM}$ EGTA (Sigma Aldrich) (prepared in high-salt Tev cleavage buffer), or $750 \mathrm{mM} \mathrm{CaCl}_{2}$ (Sigma Aldrich) (prepared in high-salt Tev cleavage buffer), with either: $89.1 \mu \mathrm{M}$ EF1p_mFAP2b and 1.40 $\mu$ M DFHBI (Supplementary Fig. 9a); $8.50 \mu \mathrm{M}$ 
EF1p_mFAP2a and $600 \mathrm{nM}$ DFHBI (Supplementary Fig. 9b); $51.4 \mu \mathrm{M}$ EF1p2_mFAP2b and $5.40 \mu$ M DFHBI (Supplementary Fig. 9c); $900 \mathrm{nM} \mathrm{EF1p2 \_ mFAP2a} \mathrm{and}$ $700 \mathrm{nM}$ DFHBI (Supplementary Fig. 9d); $47.4 \mu \mathrm{M}$ EF1p3_mFAP2b and $1.70 \mu \mathrm{M}$ DFHBI (Supplementary Fig. 9e); $30.6 \mu \mathrm{M}$ EF1p3_mFAP2a and $800 \mathrm{nM}$ DFHBI (Supplementary Fig. 9f); $107 \mu \mathrm{M}$ EF1n_mFAP2b and $2.50 \mu \mathrm{M}$ DFHBI (Supplementary Fig. 9g); $32.7 \mu \mathrm{M}$ EF1n_mFAP2a and $1.30 \mu \mathrm{M}$ DFHBI (Supplementary Fig. 9h); $47.7 \mu$ M EF1n2_mFAP2b and 13.8 $\mu$ M DFHBI (Supplementary Fig. 9i); $30.6 \mu \mathrm{M}$ EF1n2_mFAP2a and 2.40 $\mu \mathrm{M}$ DFHBI (Supplementary Fig. 9j); $125 \mu \mathrm{M}$ EF1n3_mFAP2b and 1.60 $\mu \mathrm{M}$ DFHBI (Supplementary Fig. 9k); $13.5 \mu \mathrm{M}$ EF1n3_mFAP2a and $700 \mathrm{nM}$ DFHBI (Supplementary Fig. 9l); $83.5 \mu \mathrm{M}$ EF2n_mFAP2b and 5.00 $\mu$ M DFHBI (Supplementary Fig. 9m); $6.90 \mu \mathrm{M}$ EF2n_mFAP2a and 1.20 $\mu$ M DFHBI (Supplementary Fig. 9n); $7.10 \mu \mathrm{M}$ EF2n2_mFAP2b and $7.30 \mu \mathrm{M}$ DFHBI (Supplementary Fig. 9o); $1.60 \mu \mathrm{M}$ EF2n2_mFAP2a and $1.30 \mu \mathrm{M}$ DFHBI (Supplementary Fig. 9p); $21.1 \mu \mathrm{M}$ EF2n3_mFAP2b and 13.7 $\mu$ M DFHBI (Supplementary Fig. 9q); $1.50 \mu \mathrm{M}$ EF2n3_mFAP2a and $4.60 \mu \mathrm{M}$ DFHBI (Supplementary Fig. 9r); $14.7 \mu \mathrm{M}$ EF4n_mFAP2b and $14.5 \mu \mathrm{M}$ DFHBI (Supplementary Fig. 9s); or 5.60 $\mu \mathrm{M}$ EF4n_mFAP2a and 31.4 $\mu \mathrm{M}$ DFHBI (Supplementary Fig. 9t).

EF2n_mFAP2b DFHBI titration versus $\mathbf{C a}^{2+}$ titration heatmap. $\mathrm{EF} 2 \mathrm{n} \_\mathrm{mFAP} 2 \mathrm{~b}$ was produced by large-scale protein purification ${ }^{49}$ and aliquoted to a final concentration of $500 \mathrm{nM}$ in eleven serial dilutions ( $\sqrt{10}$ dilution factor) of $\mathrm{CaCl}_{2}$ starting from $4.50 \mathrm{mM} \mathrm{CaCl} 2$ along columns, and eight serial dilutions $(\sqrt{10}$ dilution factor) of DFHBI starting from $500 \mu \mathrm{M}$ DFHBI along rows, at final volumes of $25.0 \mu \mathrm{L}$ in flat bottom, black polystyrene, non-binding surface 96 -well half-area microplates (Corning 3686). Fluorescence endpoints were measured on a Synergy Neo2 hybrid multi-mode reader using excitation wavelength $\lambda_{\mathrm{ex}}=484 \mathrm{~nm}$ and emission wavelength $\lambda_{\mathrm{em}}=508 \mathrm{~nm}$. Raw data (without background subtraction) were normalized from 0 to 1 and reported (Supplementary Fig. 14c, top row).

Split mFAP titration assays. To measure fluorescence intensities in a proteinfragment complementation assay ${ }^{12,13,52}$ (Supplementary Fig. 20b), fluorescence was measured on a Synergy Neo2 hybrid multi-mode reader (BioTek) in flat bottom, black polystyrene, non-binding surface 96 -well half-area microplates (Corning 3686). In technical triplicate, $12.0 \mu \mathrm{L}$ of each split mFAP fragment covalently fused to maltose-binding protein (MBP) was mixed to an equimolar concentration supplemented with $1.00 \mu \mathrm{L}$ of $1.25 \mathrm{mM}$ DFHBI-1T (Lucerna) at 25.0 $\mu \mathrm{L}$ final volumes per well. Fluorescence endpoints were measured using excitation wavelength $\lambda_{\mathrm{ex}}=478 \mathrm{~nm}$ and emission wavelength $\lambda_{\mathrm{em}}=520 \mathrm{~nm}$. In technical triplicate, background fluorescence endpoints of wells with identical chromophore concentrations lacking protein (substituted for equivalent volumes of high-salt Tev cleavage buffer) were measured, and the mean fluorescence endpoints were subtracted from the mean fluorescence endpoints of samples containing protein.

Split mFAP fragment affinities (Supplementary Fig. 20d-g) were estimated by preparing MBP-tagged split mFAP fragments by large-scale protein purification ${ }^{49}$ in high-salt Tev cleavage buffer, with $25.0 \mu \mathrm{M}$ DFHBI-1T final concentration at $28.0 \mu \mathrm{L}$ final volumes per well in flat bottom, black polystyrene, non-binding surface 96-well half-area microplates (Corning 3686). Three microliters of either $132 \mu \mathrm{M} \mathrm{m} 12,122 \mu \mathrm{M} \mathrm{m} 14,101 \mu \mathrm{M} \mathrm{m} 16$, or $84.9 \mu \mathrm{M} \mathrm{m} 17$ in high-salt Tev cleavage buffer was mixed with $3.00 \mu \mathrm{L}$ of $150 \mu \mathrm{M}$ DFHBI-1T in high-salt Tev cleavage buffer. For each split mFAP fragment, $12.0 \mu \mathrm{L}$ of the complementary split mFAP fragment in high-salt Tev cleavage buffer (the titrant) was mixed in from eleven serial dilutions ( $\sqrt{10}$ dilution factor) starting from $422 \mu \mathrm{M} \mathrm{m} 38,33.0 \mu \mathrm{M} \mathrm{m} 58,348$ $\mu \mathrm{M} \mathrm{m} 78$, or $531 \mu \mathrm{M} \mathrm{m} 8$ stock solutions, respectively, including a twelfth condition without titrant. Fluorescence endpoints were measured on a Synergy Neo2 hybrid multi-mode reader (BioTek) using excitation wavelength $\lambda_{\mathrm{ex}}=468 \mathrm{~nm}$ and emission wavelength $\lambda_{\mathrm{em}}=530 \mathrm{~nm}$. For each titration, the fluorescence intensity of the condition without titrant was subtracted from the fluorescence intensities of samples containing titrant, then the background-subtracted data was normalized from 0 to 1 . In collecting fluorescence excitation and emission spectra (Supplementary Fig. 20c), the conditions with the highest protein concentrations and $25.0 \mu \mathrm{M}$ DFHBI-1T were used. Excitation spectra were measured using excitation wavelengths in the range $\lambda_{\mathrm{ex}}=350-498 \mathrm{~nm}$ and emission wavelength $\lambda_{\mathrm{em}}=530 \mathrm{~nm}$, and emission spectra were measured using excitation wavelength $\lambda_{\mathrm{ex}}=468 \mathrm{~nm}$ and emission wavelengths in the range $\lambda_{\mathrm{em}}=500-650 \mathrm{~nm}$.

Fluorescence excitation and emission spectra of conditions without the addition of the complementary split mFAP fragment were measured and used for background subtraction at the corresponding wavelengths.

For titrating BCLXL_m58 into m14_aBCLXL (Fig. 6b), m14_aBCLXL and BCLXL_m58 were prepared by large-scale protein purification ${ }^{49}$ in high-salt Tev cleavage buffer. Fluorescence endpoints were measured on a Synergy Neo2 hybrid multi-mode reader (BioTek) in flat bottom, black polystyrene, non-binding surface 384-well microplates (Corning 4514) using fluorescence excitation wavelength $\lambda_{\mathrm{ex}}=468 \mathrm{~nm}$ and fluorescence emission wavelength $\lambda_{\mathrm{em}}=530 \mathrm{~nm}$. Nine wells each with $3.90 \mu \mathrm{L}$ of $19.6 \mu \mathrm{M}$ m14_aBCLXL and $2.20 \mu \mathrm{L}$ of $114 \mu \mathrm{M}$ DFHBI-1T were prepared, and $3.90 \mu \mathrm{L}$ of either high-salt Tev cleavage buffer or BCLXL_m58 was aliquoted per well to reach final concentrations of $0 \mu \mathrm{M}, 251 \mathrm{nM}, 501 \mathrm{nM}, 1.00 \mu \mathrm{M}$, $2.01 \mu \mathrm{M}, 4.01 \mu \mathrm{M}, 8.02 \mu \mathrm{M}, 16.0 \mu \mathrm{M}$, or $32.1 \mu \mathrm{M}$ BCLXL_m58, with $25.0 \mu \mathrm{M}$ DFHBI-1T and 7.64 $\mu \mathrm{M}$ m14_aBCLXL in $10.0 \mu \mathrm{L}$ final volumes per well. Fluorescence intensities were measured after $2847 \mathrm{~s}$ of double orbital shaking in the dark. Fluorescence from the $0 \mu \mathrm{M}$ BCLXL_m58 condition was subtracted from each condition, and the background-subtracted fluorescence in relative fluorescence units (RFU), $F$, was normalized by the formula:

$$
\text { Norm.Fluorescense }=\frac{F-F_{\min }}{F_{\max }-F_{\min }},
$$

where $F_{\min }(\mathrm{RFU})$ was the minimum fluorescence intensity, and $F_{\max }(\mathrm{RFU})$ was the fit to a constant function using non-linear least squares fitting of the fluorescence intensities of the four highest BCLXL_m58 concentrations. Using a bimolecular association model:

$$
\text { BCLXL_m58 + m14_aBCLXL } \underset{k_{2}}{\stackrel{k_{1}}{\rightleftharpoons}} \text { BCLXL_m58-m14_aBCLXL, }
$$

it can be shown that:

$$
\begin{aligned}
& {[\text { BCLXL_m58-m14_aBCLXL }]=0.5 \cdot\left(\left[B C L X L \_m 58\right]_{\text {total }}+\left[\mathrm{m}_{14} \_\mathrm{aBCLXL}\right]_{\text {total }}+K_{\mathrm{d}}\right)} \\
& -0.5 \cdot \sqrt{\left(-\left[\mathrm{BCLXL} \_\mathrm{m} 58\right]_{\text {total }}-\left[\mathrm{m}_{1} \_\mathrm{aBCLXL}\right]_{\text {total }}-K_{\mathrm{d}}\right)^{2}-\left(4 \cdot\left[\mathrm{BCLXL} \_\mathrm{m} 58\right]_{\text {total }} \cdot\left[\mathrm{m} 14 \_\mathrm{aBCLXL}\right]_{\text {total }}\right)},
\end{aligned}
$$

where $\frac{k_{2}}{k_{1}}=K_{\mathrm{d}}=K_{\mathrm{d}}^{\mathrm{BCLXL} \_\mathrm{m} 58-\mathrm{m} 14 \_ \text {aBCLXL}}$. The theoretical maximum fluorescent complex concentration, [BCLXL_m58-m14_aBCLXL $]_{\max }$, is reached at excess $[\text { BCLXL_m58 }]_{\text {total }}$, taken at $[\text { BCLXL_m58 }]_{\text {excess }}=10.0 \mathrm{M}$. Similarly, it can also be shown that:

$$
\begin{aligned}
& {[\text { BCLXL_m58-m14_aBCLXL }]_{\max }=0.5 \cdot\left(\left[B C L X L \_m 58\right]_{\text {excess }}+\left[\mathrm{m} 14 \_a B C L X L\right]_{\text {total }}+K_{\mathrm{d}}\right)} \\
& -0.5 \cdot \sqrt{\left(-\left[B C L X L \_m 58\right]_{\text {excess }}-\left[\mathrm{m} 14 \_\mathrm{aBCLXL}\right]_{\text {total }}-K_{\mathrm{d}}\right)^{2}-\left(4 \cdot\left[\mathrm{BCLXL} \_\mathrm{m} 58\right]_{\text {exeess }} \cdot\left[\mathrm{m} 14 \_\mathrm{aBCLXL}\right]_{\text {total }}\right)} \cdot
\end{aligned}
$$

As fluorescent complexes only form with the folded fraction of m14_aBCLXI $p_{\text {folded }}$, under the condition that $\left[\mathrm{m} 14 \_\mathrm{aBCLXL}\right]_{\text {folded }}=p_{\text {folded }} \cdot 7.64 \mu \mathrm{M}$ is the $\left[\mathrm{m} 14_{-} \mathrm{aBCLXL}\right]_{\text {total }}$, we fit $p_{\text {folded }}$ as a free parameter to the normalized fluorescence intensity with the formula:

$$
\frac{F-F_{\min }}{F_{\max }-F_{\min }}=\frac{[\text { BCLXL_m58-m14_aBCLXL }]}{[\text { BCLXL_m58-m14_aBCLXL }]_{\max }},
$$

where:

$$
K_{\mathrm{d}}^{\mathrm{BCLXL} \_\mathrm{m} 58-\mathrm{m} 14 \_\mathrm{aBCLXL}}=K_{\mathrm{d}}^{\mathrm{BCLXL}-\mathrm{aBCLXL}} \cdot K_{\mathrm{d}}^{\mathrm{m} 14-\mathrm{m} 58}=1.23 \cdot 10^{-13} \mathrm{M},
$$

because the aBCLXL domain of m14_aBCLXL associates with the binding cleft of the BCLXL domain of BCLXL_m58 with the previously reported ${ }^{37}$ BCLXL-aBCLXL thermodynamic dissociation constant of $K_{\mathrm{d}}^{\mathrm{BCLXL}-\mathrm{aBCLXL}}=$ $5.59 \cdot 10^{-9} \mathrm{M}$, and the m14 domain of m14 aBCLXL associates with the m58 domain of BCLXL_m58 with the m14-m58 thermodynamic dissociation constant taken as $K_{\mathrm{d}}^{\mathrm{m} 14-\mathrm{m} 58}=22.0 \cdot 10^{-6} \mathrm{M}$ in $25.0 \mu \mathrm{M}$ DFHBI-1T (Supplementary Fig. 20e), under an approximation that the BCLXL_m58-m14_aBCLXL interaction energy comprises only the BCLXL-aBCLXL and $\mathrm{m} 14-\mathrm{m} 58$ interaction energies:

$$
\Delta \mathrm{G}^{\text {BCLXL_m58-m14_aBCLXL }}=\Delta \mathrm{G}^{\text {BCLXL-aBCLXL }}+\Delta \mathrm{G}^{\mathrm{m} 14-\mathrm{m} 58},
$$

where $\Delta \mathrm{G}$ is the change in Gibbs free energy upon the superscripted protein-protein interaction in $25.0 \mu \mathrm{M}$ DFHBI-1T. Non-linear least squares fitting yields $p_{\text {folded }}=0.532 \pm 0.0160$, and therefore the reported $\left[\mathrm{m} 14 \_\mathrm{aBCLXL}\right]_{\text {folded }}=$ $4.06 \mu \mathrm{M}$ (Fig. 6b). The error estimate is s.d. of the fit.

Split mFAP temporal assays. In temporally monitoring fluorescence intensities (Fig. 6d), fluorescence was measured on a Synergy Neo2 hybrid multi-mode reader (BioTek) in flat bottom, black polystyrene, non-binding surface 96-well microplates (Corning 3650) using excitation wavelength $\lambda_{\mathrm{ex}}=468 \mathrm{~nm}$ and emission wavelength $\lambda_{\mathrm{em}}=530 \mathrm{~nm}$. aBCL2, aBFL1, aBCLXL, m14_aBCL2, m14_aBFL1, m14_aBCLXL, BCL2_m58, BFL1_m58, and BCLXL_m58 were prepared by large-scale protein purification ${ }^{49}$ in high-salt Tev cleavage buffer. Two wells each with $36.0 \mu \mathrm{L}$ of either aBCL2, aBFL1, or aBCLXL, $36.0 \mu \mathrm{L}$ of either BCL2_m58, BFL1_m58, or BCLXL_m58, and $12.0 \mu \mathrm{L}$ of $250 \mu \mathrm{M}$ DFHBI-1T were prepared with matched cognate-binding partners, and samples were mixed by double orbital shaking at room temperature for $30 \mathrm{~min}$ in the dark. Subsequently, $36.0 \mu \mathrm{L}$ of high-salt Tev cleavage buffer was aliquoted into the first of the two wells (negative control group), and $36.0 \mu \mathrm{L}$ of either m14_aBCL2, m14_aBFL1, or m14_aBCLXL was aliquoted into the second of the two wells (experimental group) with matched cognate-binding partners, respectively. Fluorescence intensities were measured every $30 \mathrm{~s}$ between $5 \mathrm{~s}$ double orbital shake steps to mix the samples for $1200 \mathrm{~s}$. Final sample conditions were $2.79 \mu \mathrm{M}$ of aBCL2, m14_aBCL2, and BCL2_m58, $2.48 \mu \mathrm{M}$ of aBFL1, m14_aBFL1, and BFL1_m58, $3.88 \mu \mathrm{M}$ of aBCLXL, m14_aBCLXL, and BCLXL_m58, and $25.0 \mu \mathrm{M}$ DFHBI-1T for all conditions in 120 $\mu \mathrm{L}$ final volumes per well. For each condition, fluorescence fold-change was calculated as:

$$
\frac{\Delta F}{F_{0}}=\frac{F-F_{0}}{F_{0}},
$$

where $F(\mathrm{RFU})$ is the fluorescence intensity per measurement and $F_{0}(\mathrm{RFU})$ is the fluorescence intensity of the first measurement, then fluorescence fold-change was fit to a monophasic exponential function using non-linear least squares fitting (Fig. 
6d). In collecting fluorescence excitation and emission spectra after reaching equilibrium (Supplementary Fig. 21a), fluorescence excitation spectra were measured using excitation wavelengths in the range $\lambda_{\mathrm{ex}}=350-530 \mathrm{~nm}$ and emission wavelength $\lambda_{\mathrm{em}}=562 \mathrm{~nm}$, and emission spectra were measured using excitation wavelength $\lambda_{\mathrm{ex}}=438 \mathrm{~nm}$ and emission wavelengths in the range $\lambda_{\mathrm{em}}=470-650$ $\mathrm{nm}$, and the normalized spectra reported without background subtraction.

In temporally monitoring fluorescence intensities (Fig. $6 \mathrm{f}$ ), fluorescence was measured on a Synergy Neo2 hybrid multi-mode reader (BioTek) in flat bottom, black polystyrene, non-binding surface 96-well half-area microplates (Corning 3686) using excitation wavelength $\lambda_{\mathrm{ex}}=478 \mathrm{~nm}$ and emission wavelength $\lambda_{\mathrm{em}}=530 \mathrm{~nm}$. m14_aBFL1, BCL2_m58, and aBCL2 were prepared by large-scale protein purification ${ }^{49}$ in high-salt Tev cleavage buffer. Three wells of $2.22 \mu \mathrm{M}$ m14_aBFL1 with $2.22 \mu \mathrm{M}$ BCL2_m58 and 27.8 $\mu \mathrm{M}$ DFHBI-1T in high-salt Tev cleavage buffer at final volumes of $45.0 \mu \mathrm{L}$ were prepared and mixed by double orbital shaking at room temperature for $20 \mathrm{~min}$ in the dark. Subsequently, $5.00 \mu \mathrm{L}$ of either $100 \mu \mathrm{M}$ aBCL2, $40.0 \mu \mathrm{M}$ aBCL2, or high-salt Tev cleavage buffer was aliquoted per well, respectively, and fluorescence intensities measured every $12 \mathrm{~s}$ between $5 \mathrm{~s}$ double orbital shake steps to mix the samples for 2,604 s. Final sample conditions were $25.0 \mu \mathrm{M}$ DFHBI-1T, 2.00 $\mu \mathrm{M}$ m14_aBFL1, $2.00 \mu \mathrm{M}$ BCL2_m58 and either $10.0 \mu \mathrm{M}, 4.00 \mu \mathrm{M}$, or $0 \mu \mathrm{M}$ aBCL2 in $50.0 \mu \mathrm{L}$ final volumes per well. For each condition, fluorescence fold-change was calculated by Eq. (11) where $F(\mathrm{RFU})$ is the fluorescence intensity per measurement and $F_{0}(\mathrm{RFU})$ is the fluorescence intensity of the first measurement, then fluorescence foldchange was fit to a monophasic exponential function using non-linear least squares fitting (Fig. 6f). In collecting fluorescence excitation and emission spectra after reaching equilibrium (Supplementary Fig. 21b), fluorescence excitation spectra were measured using excitation wavelengths in the range $\lambda_{\mathrm{ex}}=350-530 \mathrm{~nm}$ and emission wavelength $\lambda_{\mathrm{em}}=570 \mathrm{~nm}$, and fluorescence emission spectra were measured using excitation wavelength $\lambda_{\mathrm{ex}}=430 \mathrm{~nm}$ and emission wavelengths in the range $\lambda_{\mathrm{em}}=470-750 \mathrm{~nm}$, and the normalized spectra reported without background subtraction.

Circular dichroism. Circular dichroism (CD) measurements were recorded at $25^{\circ} \mathrm{C}$ in a $1 \mathrm{~mm}$ cuvette on an AVIV model $420 \mathrm{CD}$ spectrometer (Biomedical, Inc.). mFAP2 and mFAP2.1 were purified by large-scale protein purification and SEC purification ${ }^{49}$ in phosphate-buffered saline (PBS) $(25.0 \mathrm{mM}$ phosphate, $150 \mathrm{mM} \mathrm{NaCl}, \mathrm{pH} 7.40$ ), and far-ultraviolet $\mathrm{CD}$ wavelength scans recorded from $195 \mathrm{~nm}$ to $260 \mathrm{~nm}$. mFAP2 was measured at $0.441 \mathrm{mg} \mathrm{mL}^{-1}$ and mFAP2.1 at $0.500 \mathrm{mg} \mathrm{mL}^{-1}$ in $\mathrm{Na}_{2} \mathrm{HPO}_{4}$-citrate buffer with $\mathrm{pH}$ adjusted to $7.75,3.96$, or 2.93 using $\mathrm{NaOH}$ and $\mathrm{HCl}$. The reported data was background-subtracted using buffer only contols (Supplementary Fig. 1b, c).

EF4n_mFAP2b (Supplementary Fig. 8) was purified by large-scale protein purification and SEC purification ${ }^{49}$ in Dulbecco's Phosphate-Buffered Saline without calcium or magnesium (DPBS) (Thermo Scientific). A small amount of Chelex 100 was added to the protein sample and nutated overnight at $4{ }^{\circ} \mathrm{C}$. A stock solution of $1.00 \mathrm{mM} \mathrm{CaCl}_{2}$ was prepared in DPBS pre-treated with a small amount of Chelex 100 overnight. Far-ultraviolet CD wavelength scans and thermal denaturations were performed with $0.500 \mathrm{mg} \mathrm{mL}^{-1}$ protein in either $100 \mu \mathrm{M} \mathrm{CaCl}_{2}$ or DPBS, both using Chelex 100 pre-treated DPBS. Far-ultraviolet CD wavelength scans from $195 \mathrm{~nm}$ to $260 \mathrm{~nm}$ were recorded at $25^{\circ} \mathrm{C}$, and thermal denaturation was monitored at $220 \mathrm{~nm}$ wavelength from $25^{\circ} \mathrm{C}$ to $95^{\circ} \mathrm{C}$ at $2{ }^{\circ} \mathrm{C}$ evenly spaced intervals. Reported data are background-subtracted from the corresponding $100 \mu \mathrm{M} \mathrm{CaCl}_{2}$ or DPBS buffer measurements without protein.

Quantum yield measurements. Protein preparation: mFAP2a, mFAP2b, and mFAP10 were produced by large-scale protein purification and SEC purification ${ }^{49}$, and dialyzed overnight into DPBS that was adjusted to $\mathrm{pH} 7.40$ using $\mathrm{NaOH}$.

Chromophore preparation: DFHBI (Lucerna) and DFHBI-1T (Lucerna) were dissolved to $20.0 \mathrm{mM}$ in $100 \%$ DMSO, and diluted in DPBS (pH 7.40) to measure absorbances on a Jasco V-750 spectrophotometer at peak absorbance wavelengths $(417 \mathrm{~nm}$ for DFHBI and $422 \mathrm{~nm}$ for DFHBI-1T). Following background subtraction of identical buffer without chromophore, Beer's Law was used to calculate the molar chromophore concentrations of the stock solutions using previously reported extinction coefficients ${ }^{4}$.

Preparation of protein-chromophore complexes: For quantum yield measurements, $1.00 \mu \mathrm{M}, 836 \mathrm{nM}$, or $919 \mathrm{nM}$ chromophore solutions in DPBS $(\mathrm{pH} 7.40)$ at $4.00 \mathrm{~mL}$ final volumes were prepared for the following eight conditions: DFHBI only, DFHBI-1T only, $43.5 \mu \mathrm{M}$ 6xHis-mFAP10 with DFHBI, $43.5 \mu \mathrm{M}$ 6xHis-mFAP10 with DFHBI-1T, $134 \mu \mathrm{M}$ 6xHis-mFAP2a with DFHBI, $134 \mu \mathrm{M}$ 6xHis-mFAP2a with DFHBI-1T, $206 \mu \mathrm{M}$ 6xHis-mFAP2b with DFHBI, and $206 \mu \mathrm{M}$ 6xHis-mFAP2b with DFHBI-1T.

Extinction coefficients: Absorbance spectra of protein-chromophore complexes were first measured with a Thermo Scientific BioMate 3S UV-Vis Spectrophotometer $\left(1 \mathrm{~nm}\right.$ interval, $\left.800 \mathrm{~nm} \mathrm{~min}^{-1}\right)$. The extinction coefficients were then calculated using Beer's Law:

$$
A=\varepsilon \cdot b \cdot c,
$$

where $A$ is peak absorbance, $\varepsilon$ is extinction coefficient, $b$ is path length $(1 \mathrm{~cm})$, and $c$ is concentration $(1.00 \mu \mathrm{M}, 836 \mathrm{nM}$, or $919 \mathrm{nM})$.

Relative quantum yield: A Perkin-Elmer LS-B Luminescence Spectrophotometer $\left(10 \mathrm{~nm}\right.$ bandwidth, $1 \mathrm{~nm}$ interval, $100 \mathrm{~nm} \mathrm{~min}{ }^{-1}$ ) was used. The fluorescence emission spectra of the protein-chromophore complexes (in DPBS, pH 7.40) and reference dye Acridine Yellow G (in methanol) were first obtained, and the quantum yield was then calculated using the equation ${ }^{53}$ :

$$
\phi_{\mathrm{c}}=\phi_{\mathrm{r}} \cdot \frac{1-10^{-A_{\mathrm{r}}\left(\lambda_{\mathrm{ex}}\right)}}{1-10^{-A_{\mathrm{c}}\left(\lambda_{\mathrm{ex}}\right)}} \cdot \frac{\int F_{\mathrm{c}}(\lambda) \cdot d \lambda}{\int F_{\mathrm{r}}(\lambda) \cdot d \lambda} \cdot \frac{n_{\mathrm{c}}^{2}}{n_{\mathrm{r}}^{2}},
$$

where $\phi$ is quantum yield, $A\left(\lambda_{\mathrm{ex}}\right)$ is absorbance at the excitation wavelength $\lambda_{\text {ex }}\left(\lambda_{\text {ex }}=440 \mathrm{~nm}\right), F$ is fluorescence emission, $n$ is refractive index of the solution (1.3350 for DPBS at pH 7.40 and 1.3284 for methanol), and the subscripts "c" and " $\mathrm{r}$ " refer to the protein-chromophore complex measured and the reference dye, respectively. The reference dye Acridine Yellow G (in methanol) has a quantum yield value of 0.57 that was used ${ }^{54}$.

Absolute quantum yield: An integrating sphere instrument (Hamamatsu C992012) $(6 \mathrm{~nm}$ excitation bandwidth, $1 \mathrm{~nm}$ interval) and a high-sensitivity photonic multi-channel analyzer (Hamamatsu C10027-01) were used to measure a light emission spectrum. Absolute quantum yields were measured for solutions of protein-chromophore complexes in DPBS ( $\mathrm{pH} 7.40$ ) in which $\geq 95 \%$ of the total chromophore was occupying the protein-binding pocket (Table 1).

Protein-chromophore complex samples and control samples were excited at $\lambda_{\mathrm{ex}}=$ $440 \mathrm{~nm}$ and absolute quantum yields were calculated according to the equation:

$$
\phi_{\mathrm{c}}=\frac{f_{\mathrm{em}}}{f_{\mathrm{abs}}},
$$

where $f_{\mathrm{em}}$ is the emitted photon flux and $f_{\text {abs }}$ is the absorbed photon flux. The absolute quantum yields of the two control samples (Acridine Yellow G and fluorescein) agreed well with literature values ${ }^{54,55}$. Absolute quantum yield data was analyzed with U6039-05 PLQY measurement software.

Size-exclusion chromatography with multi-angle light scattering. Protein samples were prepared at $2.0 \mathrm{mg} \mathrm{mL}^{-1}$ and applied to a Superdex $7510 / 300 \mathrm{GL}$ column (GE Healthcare) on a LC 1200 Series HPLC machine (Agilent Technologies) for size-based separation, a Heleos detector (Wyatt Technologies) for light scattering signals, and a t-Rex detector for differential refractive index detection. Results were analyzed using ASTRA 7.2 software for weighted average molecular weight.

COS-7 cell culture and transfection. COS-7 cells (ATCC CRL-1651) were cultured in Dulbecco's modified Eagle's medium (DMEM) supplemented with 1x NEAA, 100 units $\mathrm{mL}^{-1}$ penicillin, $100 \mu \mathrm{gL}^{-1}$ streptomycin, and $10 \%$ fetal bovine serum (FBS) For transfection, cells were collected using $0.25 \%$ trypsin EDTA, and approximately 1 million cells transfected by nucleofection using $2 \mu \mathrm{g}$ of plasmid DNA (Supplementary Data 10), 18.0 $\mu \mathrm{L}$ of Lonza s.e. cell supplement, $82.0 \mu \mathrm{L}$ of Lonza s.e. nucleofection solution, and pulse code DS-120 on a Lonza 4D X Nucleofector system. Cells were seeded into ibidi $\mu$-Slide 8 -well glass bottom chambers at a density of approximately 30,000 cells well $^{-1}$ and recovered overnight at $37^{\circ} \mathrm{C}$.

cos-7 cell fixation. COS- 7 cells were treated at $37^{\circ} \mathrm{C}$ with PFA/GA fixation solution (containing $100 \mathrm{mM}$ aqueous PIPES buffer at $\mathrm{pH} 7.0,1 \mathrm{mM} \mathrm{MgCl}_{2}, 3.2 \%$ paraformaldehyde, and $0.1 \%$ gluteraldehyde) for $10 \mathrm{~min}$, reduced with $10 \mathrm{mM}$ aqueous sodium borohydride for $10 \mathrm{~min}$, then rinsed with 1x PBS $(11.9 \mathrm{mM}$ phosphates, $137 \mathrm{mM} \mathrm{NaCl}, 2.70 \mathrm{mM} \mathrm{KCl}, \mathrm{pH}$ 7.40) (Fisher Scientific \#BP399-1) for 5 min (Supplementary Fig. 4).

Epifluorescence microscopy of COS-7 cells. Conventional widefield epifluorescence imaging of cultured live COS-7 cells (Supplementary Movie 1 and Supplementary Movie 2) and fixed COS-7 cells (Supplementary Fig. 4) was performed on an inverted Nikon Ti-S microscope configured with a $60 \mathrm{x} / 1.2 \mathrm{NA}$ water-immersion objective lens (Nikon), a multiband filter set (LF405/488/532/ 635-A-000, Semrock), and a Zyla 5.5 sCMOS camera (Andor). Micro-Manager software with MM Studio and MMCore were used for acquisition. For widefield epifluorescence microscopy of fixed COS-7 cells expressing mFAP2a or mFAP2b targeted to the endoplasmic reticulum (ER) (Supplementary Fig. 4), samples were labeled with either $40.0 \mu \mathrm{M}$ DFHBI or $40.0 \mu \mathrm{M}$ DFHBI-1T in 1x PBS for at least $10 \mathrm{~min}$ before imaging. Cells were rinsed three times with $1.00 \mathrm{~mL}$ of $1 \mathrm{x}$ PBS. Samples were illuminated with $470 \mathrm{~nm}$ light at an intensity of $\sim 2 \mathrm{~W} \mathrm{~cm}^{-2}$. Exposure times were $200 \mathrm{~ms}$ and current was $500 \mathrm{~mA}$. For time-lapse widefield epifluorescence microscopy of live COS-7 cells expressing mFAP2a or mFAP2b targeted to the ER (Supplementary Movie 1, Supplementary Movie 2), cells were labeled with $40.0 \mu \mathrm{M}$ DFHBI in 1x PBS. The time-lapse movies were acquired using $200 \mathrm{~ms}$ exposure times every $5 \mathrm{~s}$ for 25 total frames, with $100 \mathrm{~mA}$ excitation current. The total acquisition duration per movie was just over $2 \mathrm{~min}$, and movie playback speeds adjusted to 5 frames $\mathrm{s}^{-1}$.

Photostability assay. COS-7 cells transfected with either pcDNA5/FRT/TO AcGFP1-sec61 $\beta$, pcDNA5/FRT/TO-mFAP2a-sec61 $\beta$, or pcDNA5/FRT/TOmFAP2b-sec61 $\beta$ (Supplementary Data 10) were fixed and imaged (Fig. 2) at $25^{\circ} \mathrm{C}$ using a Zeiss LSM-510 laser scanning confocal fluorescence microscope equipped with a Plan-Apochromat $63 \mathrm{x} / 1.4$ NA oil DIC objective lens and SP1 software. The Argon/2 $488 \mathrm{~nm}$ excitation laser was set to $50 \%$ output power (4.0 A tube current) and at $10 \%$ transmission resulting in $10.3 \mu \mathrm{W}$ laser power. The pinhole size was set 
to $98 \mu \mathrm{m}$ (1.02 Airy units). The excitation laser source passed through a HFT488 dichroic beam splitter to the specimen, and fluorescence captured through the HFT488 dichroic beam splitter to a $505 \mathrm{~nm}$ long-pass emission filter to the detector. Acquiring $740 \times 740$ pixel $\left(71.43 \mu \mathrm{m}^{2}\right)$ images with a single laser scan direction and 12-bit pixel depth resulted in a $1.13 \mathrm{~s}$ scantime and $0.8 \mu$ s pixeltime for photostability assays. For all samples, amplifier offset was set to -1.0 and amplifier gain set to 1.0. For samples expressing AcGFP1-sec61 $\beta$, detector gain was set to 500 . For samples expressing mFAP2a-sec61 $\beta$, detector gain was set to 800 for $50.0 \mu \mathrm{M}$ DFHBI labeling, 700 for $50.0 \mu \mathrm{M}$ DFHBI-1T labeling, and 900 for $500 \mathrm{nM}$ DFHBI and $500 \mathrm{nM}$ DFHBI-1T labeling. For samples expressing mFAP2b-sec61 $\beta$, detector gain was set to 700 for $50.0 \mu \mathrm{M}$ DFHBI labeling and 900 for $500 \mathrm{nM}$ DFHBI labeling. Fixed COS-7 cells expressing AcGFP1-sec61 $\beta$ were imaged in Tris buffered saline (TBS) (25.0 mM Tris, $300 \mathrm{mM} \mathrm{NaCl}, \mathrm{pH}$ 8.00), and those expressing mFAP2a-sec61 $\beta$ and mFAP2b-sec61 $\beta$ were washed ten times with $50.0 \mu \mathrm{L}$ of highsalt Tev cleavage buffer, and labeled with chromophore in high-salt Tev cleavage buffer for at least $10 \mathrm{~min}$ prior to imaging. Raw "*.lsm" data files were analyzed using linear lookup tables covering the full range of data (Fig. 2a, b). For each region of interest encompassing one or more fixed cells, normalized image intensity (Fig. 2c, d) was calculated by summing image pixel intensities per frame and dividing by the summed image pixel intensities of the first frame. Pixel intensities at the bit depth of the microscope detector were discounted. Non-linear least squares fitting of the average normalized image intensities to a monophasic exponential decay function of the form $F=h+(1-h) \cdot \mathrm{e}^{-k \cdot t}$ was used to obtain $h$ constants, the percentage at which the normalized average image intensities asymptotically plateau. The ratio of $h$ constants relative to AcGFP1 were reported as improved photostability over AcGFP1.

\section{Laser scanning confocal fluorescence microscopy of E. coli. Lemo21(DE3) E.} coli cultures expressing either mFAP2a or mFAP2b were induced at $500 \mu \mathrm{M}$ IPTG final concentration for $4 \mathrm{~h}$ at $37^{\circ} \mathrm{C}$ shaking at $250 \mathrm{rpm}$. For imaging separate cultures (Fig. 1f-i), 1.5\% agarose pads supplemented with either $10.0 \mu \mathrm{M}$ DFHBI or 10.0 $\mu \mathrm{M}$ DFHBI-1T final concentrations were each molded using six stacked microscope slides on a leveled surface ${ }^{56}$. In all, $1.00 \mathrm{~mL}$ of induced cells was aliquoted from each culture, centrifuged, the pellet resuspended in $1.00 \mathrm{~mL}$ of highsalt Tev cleavage buffer, solution pelleted again, and the pellet resuspended in either $100 \mu \mathrm{L}$ of $10.0 \mu \mathrm{M}$ DFHBI or $100 \mu \mathrm{L}$ of $10.0 \mu \mathrm{M}$ DFHBI-1T (from $2.00 \mathrm{mM}$ chromophore stock solutions in 5\% DMSO and 95\% high-salt Tev cleavage buffer), and incubated for $10 \mathrm{~min}$ at $25^{\circ} \mathrm{C}$. Two microliters of cells in chromophore solution were aliquoted onto $\sim 1 \mathrm{~cm}^{2}$ agarose pads containing the corresponding chromophore, and the agarose pad placed in $\mu$-Slide 4 Well chambers (ibidi) for imaging.

For imaging mixed cultures (Supplementary Fig. 3), induced E. coli cultures were diluted 10 -fold in $\mathrm{ddH}_{2} \mathrm{O}$ and their optical density at $600 \mathrm{~nm}$ measured using a Genesys 10S UV-Vis spectrophotometer (Thermo Scientific). Proportional volumes of cell cultures expressing either mFAP2a or mFAP2b were mixed to achieve a 1:1 ratio of cells from each culture. One-hundred microliters of this mixture was centrifuged, the pellet resuspended in $200 \mu \mathrm{L}$ of high-salt Tev cleavage buffer, solution pelleted again, and the pellet resuspended in either $10.0 \mu \mathrm{L}$ of $10.0 \mu \mathrm{M}$ DFHBI or $10.0 \mu \mathrm{L}$ of $10.0 \mu \mathrm{M}$ DFHBI-1T (from $2.00 \mathrm{mM}$ chromophore stock solutions in 5\% DMSO and 95\% high-salt Tev cleavage buffer), and incubated for $10 \mathrm{~min}$ at $25^{\circ} \mathrm{C}$. Five microliters of each cellular mixture in different chromophores was pipetted onto frosted microscope slides (Fisher Scientific) between Premium Superslip glass coverslips (Fisher Scientific).

Images were acquired on a Zeiss LSM-510 laser scanning confocal fluorescence microscope equipped with a Plan-Apochromat 63x/1.4 NA oil DIC objective lens and SP1 software. The Argon/2 $488 \mathrm{~nm}$ excitation laser was set to $50 \%$ output power $(4.0 \mathrm{~A}$ tube current) and at $10 \%$ transmission resulting in $10.3 \mu \mathrm{W}$ laser power. Pinhole size was set to $98 \mu \mathrm{m}$ (1.02 Airy units), and fluorescence was captured through a $505 \mathrm{~nm}$ long-pass emission filter. Detector gain was set to 650 , amplifier offset was set to -0.85 , and amplifier gain set to 1.0 . $740 \times 740$ pixel $\left(71.43 \mu \mathrm{m}^{2}\right)$ images (Fig. $\left.1 \mathrm{f}-\mathrm{i}\right)$ or $1480 \times 1480$ pixel $\left(142.86 \mu \mathrm{m}^{2}\right)$ images (Supplementary Fig. 3) were acquired with a single laser scan direction and 12-bit pixel depth. Raw "*.lsm" data files were analyzed using linear lookup tables covering the full range of data (Fig. If-i and Supplementary Fig. 3a, b). In Supplementary Fig. 3c, summed pixel intensities were calculated per image including the pixel intensities at the bit depth of the microscope detector.

X-ray crystallography. EF1p2_mFAP2b was produced by large-scale protein purification, 6xHis-tag removal and SEC purification ${ }^{49}$ in TBS. Purified protein was mixed with excess DFHBI (resuspended in 100\% DMSO), while keeping the final DMSO concentration $<1 \%$, followed by addition of $5.00 \mathrm{mM} \mathrm{CaCl}_{2}$ final concentration. The EF1p2_mFAP2b-DFHBI complex was then concentrated to $\sim 25 \mathrm{mg} \mathrm{mL}^{-1}$, and initially tested for crystallization via sparse matrix screens in $96-$ well sitting drops using a mosquito (TTP LabTech). A single crystal was obtained in a $200 \mathrm{~nL}$ drop from $100 \mathrm{mM}$ HEPES pH 7.50 and 25\% PEG 3350 (Index, Hampton Research). The drop was flooded with reservoir solution plus $2.00 \mathrm{mM}$ DFHBI and $20 \%$ ethylene glycol then flash frozen in liquid nitrogen. Data was collected with a home-source rotating anode on a Saturn $944+$ CCD and processed in HKL2000 ${ }^{57}$. For phasing and refinement, structures were solved by Molecular Replacement with Phaser via phenix ${ }^{58,59}$ using a mFAP2b design model from
Rosetta $^{23}$ with appropriate residue side-chains cut back to $\mathrm{C}_{\alpha}$ atoms, and DFHBI and loop7 residues removed. The structure was then built and refined using $\operatorname{Coot}^{60}$ and phenix ${ }^{61}$, respectively (Supplementary Table 3 ).

Molecular dynamics simulations. Chain $\mathrm{B}$ of the refined

EF1p2_mFAP2b-DFHBI-Ca ${ }^{2+}$ co-crystal structure (PDB accession code 6OHH) was used as the starting point for molecular dynamics (MD) simulations. The other three system conditions were obtained by removing the coordinates of the $\mathrm{Ca}^{2+}$ ion and DFHBI ligand (Apo), or either that of the ion or of the ligand (DFHBIbound and $\mathrm{Ca}^{2+}$-bound conditions, respectively). Missing side-chains were added using Schrödinger's Maestro (version 10.4, Schrödinger, LLC, New York, NY) and all crystallographic waters were kept. Protonation states at $\mathrm{pH} 7$ were assigned using Maestro's PROPKA. The accessible protein cavities left by the removal of the ligand in the Apo and $\mathrm{Ca}^{2+}$-bound systems were hydrated with Dowser ${ }^{62}$. Proteins were solvated in TIP3P water boxes ${ }^{63}$ with a buffer distance of $16 \AA$ to the box edges and $\mathrm{NaCl}$ ions were added to provide charge neutrality at a total concentration of $150 \mathrm{mM}$. The Amber14SB force field ${ }^{64,65}$ was used for the protein and $\mathrm{NaCl}$. The DFHBI ligand was parametrized using Antechamber and the generalized Amber Force Field (GAFF) ${ }^{66,67}$, with geometry optimization performed with Gaussian $09^{68} . \mathrm{Ca}^{2+}$ parameters were obtained from Bradbrook et al. ${ }^{69}$.

The systems were minimized in five stages with increasing number of unconstrained atoms (proton only, solvent, ligand, side-chains, and the full system) totaling 13,000 steps of steepest descent and conjugate gradient methods. This was followed by equilibration involving an initial heating to $100 \mathrm{~K}$ at constant volume for $50 \mathrm{ps}$ followed by heating to $298 \mathrm{~K}$ at a constant pressure of $1 \mathrm{bar}$ for $200 \mathrm{ps}$. The systems were further equilibrated at $298 \mathrm{~K}$ and 1 bar for $2.25 \mathrm{~ns}$. Production runs were performed using GPU accelerated Amber14 $4^{65,70}$ at 1 bar and $298 \mathrm{~K}$ with periodic boundary conditions and a $2 \mathrm{fs}$ timestep, with non-bonded short-range interactions evaluated within a cutoff of $10 \AA$. Each of the four system conditions were simulated in three independent $500 \mathrm{~ns}$ replicates. The first $100 \mathrm{~ns}$ of each production run were discounted from analysis to allow for adequate structural relaxation from the starting conformation. The trajectories were visualized in $\mathrm{VMD}^{71}$ and aligned to the co-crystal protein structure. The protein backbone atom coordinates were used to probe the conformational free energy landscape using PyEMMA $^{72}$ and in-house scripts. The loop7 residue coordinates of all simulations were jointly clustered into 25 clusters, resulting in whole protein cluster centroids with an average backbone heavy atom root mean square deviation (RMSD) of $2.49 \AA$ to each other. Clustering was performed on loop7 backbone coordinates using PyEMMA's $\mathrm{k}$-means algorithm, and each cluster centroid structure was defined as the structure, which minimized the sum of the RMSD values to all other cluster members. CPPTRAJ ${ }^{73}$ and MDTraj ${ }^{74}$ were used for RMSF and RMSD analysis.

HEK293 cell transfections. Plasmid DNAs (Supplementary Data 15 and Supplementary Data 17) were purified in $\mathrm{ddH}_{2} \mathrm{O}$ for transfections. pGP-CMVGCaMP6f was a gift from Douglas Kim \& GENIE Project (Addgene plasmid \#40755; [http://n2t.net/addgene:40755]; RRID: Addgene_40755). Wild-type HEK293 cells (ATCC CRL-1573) were cultured on 24-well plates in DMEM media (4.5 g L L-1 D-glucose, L-glutamine; ThermoFisher \#11965-092) supplemented with $10 \%$ FBS and $1 \%$ Penicillin/Streptomycin in a $5 \% \mathrm{CO}_{2}$ atmosphere at $37^{\circ} \mathrm{C}$. Cells were seeded into 24 -well plates (ThermoFisher \#FB012929) at 100,000 cells well ${ }^{-1}$. Twenty-four hours after seeding, cell media was aspirated and replaced with $200 \mu \mathrm{L}$ of DMEM media. Lipofectamine 3000 (ThermoFisher Scientific) reagents were prepared according to the manufacturer's instructions by mixing $1.50 \mu \mathrm{L}$ of Lipofectamine 3000 reagent diluted in $25.0 \mu \mathrm{L}$ of OPTI-MEM with $1 \mu \mathrm{g}$ of plasmid DNA diluted in $25.0 \mu \mathrm{L}$ OPTI-MEM and $2.00 \mu \mathrm{L}$ of P3000 reagent, allowing for formation of DNA-lipid complexes for $\sim 10 \mathrm{~min}$ after combination of the two mixtures. Reagent volumes were increased for the number of wells to be transfected. Fifty microliters of complexed Lipofectamine reagents were pipetted into each well, cells were incubated for $30 \mathrm{~min}$, and the volume in each well was raised to $750 \mu \mathrm{L}$ with DMEM media. Approximately $6 \mathrm{~h}$ after transfection, media was replaced with $1.00 \mathrm{~mL}$ of DMEM media. Cells were incubated for $48 \mathrm{~h}$ to allow for protein expression.

HEK293 cell surface-displayed $\mathrm{Ca}^{2}+$ titrations. $\mathrm{Ca}^{2+}$ titration protocol: A stock solution of $20.0 \mathrm{mM}$ DFHBI was prepared in $100 \%$ anhydrous DMSO. Two days after the transfection of pDisplay-EF1p_mFAP2b, each 24-well plate well was rinsed twice with pre-warmed $\mathrm{Ca}^{2+}$-deficient Tyrode's solution $(124 \mathrm{mM} \mathrm{NaCl}$, $2 \mathrm{mM} \mathrm{KCl}, 2 \mathrm{mM} \mathrm{MgCl}, 10 \mathrm{mM}$ HEPES and $10 \mathrm{mM}$ glucose, $\mathrm{pH}$ between 7.3 and 7.4 with $\mathrm{NaOH}$ ), then filled with $200 \mu \mathrm{L}$ of the same solution containing $7.00 \mu \mathrm{M}$ DFHBI (approximately $\left(K_{\mathrm{d}}^{+} \cdot K_{\mathrm{d}}^{-}\right)^{1 / 2}$ for EF1p_mFAP2b; Supplementary Table 2). Cells were incubated $\left(5 \% \mathrm{CO}_{2}\right.$ at $\left.37^{\circ} \mathrm{C}\right)$ in the dark for $5 \mathrm{~min}$ before the $\mathrm{Ca}^{2+}$ titration to allow DFHBI to reach equilibrium with the sensor. Cells were imaged at 5 frames s ${ }^{-1}$ for 1 min with a sCMOS camera (Photometrics Prime95B) and a 20x magnification lens (Leica HCX PL FLUOTAR L 20x/0.40 NA CORR). The cells were continually illuminated at $7.65 \mathrm{~mW} \mathrm{~cm}-2$ with a LumenCor Light Engine (Semrock filters: Excitation 474/27 nm; Emission 520/35 nm). Twenty seconds into the imaging experiment, a syringe pump (Harvard Apparatus Pump 11 Elite) was triggered by a TTL pulse. Two-hundred microliters of $20 \mathrm{mM} \mathrm{Ca}^{2+}$ Tyrode's solution $\left(84 \mathrm{mM} \mathrm{NaCl}, 2 \mathrm{mM} \mathrm{KCl}, 2 \mathrm{mM} \mathrm{MgCl} 2,20 \mathrm{mM} \mathrm{CaCl}_{2}, 10 \mathrm{mM}\right.$ HEPES 
and $10 \mathrm{mM}$ glucose, $\mathrm{pH}$ between 7.3-7.4 with $\mathrm{NaOH}$ ) with the identical DFHB concentration was added at a rate of $2 \mathrm{~mL} \mathrm{~min}^{-1}$ to the wells. In technical triplicate, the final conditions of each well were $400 \mu \mathrm{L}$ volume and $10 \mathrm{mM} \mathrm{Ca}^{2+}$, with constant 7.00 $\mu \mathrm{M}$ DFHBI concentration throughout the experiment. "After Photobleaching" (see Fig. 5d) cells in a different region of interest (ROI) were illuminated for the same duration and imaging conditions used for each imaging experiment, prior to the titration experiment.

Data analysis: Regions of interest (ROIs) surrounding single cells from three technical replicates were hand-drawn in ImageJ software ${ }^{75}$. For each cellular ROI in each frame the average fluorescence was calculated in ImageJ. In order to perform background subtraction, cellular ROIs were then moved proximal to the original cell to an ROI where there was no fluorescence. Each cellular ROIs average fluorescence had the average background intensity subtracted for each frame. Following background subtraction, fluorescence fold-change was calculated by Eq. (11) where $F$ is the background-subtracted average fluorescence per ROI per frame, and $F_{0}$ is the background-subtracted average baseline fluorescence as measured $1 \mathrm{~s}$ prior to the $\mathrm{Ca}^{2+}$ titration ${ }^{29}$ (Fig. $5 \mathrm{~d}$ ).

HEK293 cell acetylcholine stimulations. Stimulation protocol: A stock solution of $20.0 \mathrm{mM}$ DFHBI was prepared in $100 \%$ anhydrous DMSO. Media was aspirated from the wells and the cells were rinsed once with $200 \mu \mathrm{L}$ of Tyrode's solution $(120$ $\mathrm{mM} \mathrm{NaCl}, 2 \mathrm{mM} \mathrm{KCl}, 2 \mathrm{mM} \mathrm{MgCl} 2,2 \mathrm{mM} \mathrm{CaCl}, 10 \mathrm{mM}$ HEPES, $10 \mathrm{mM}$ glucose, $\mathrm{pH}$ adjusted to 7.3-7.4 using $\mathrm{NaOH}$ ). Cells were placed in $750 \mu \mathrm{L}$ Tyrode's solution supplemented with $20.0 \mu \mathrm{M}$ DFHBI for EF2n_mFAP2a-expressing cells, $43.3 \mu \mathrm{M}$ DFHBI for EF4n_mFAP2a-expressing cells, and $43.3 \mu \mathrm{M}$ DFHBI for EF4n_mFAP2b-expressing cells. GCaMP6f-expressing cells were placed in $750 \mu \mathrm{L}$ Tyrode's solution. Cells were placed in the dark at $37^{\circ} \mathrm{C}$ for $30 \mathrm{~min}$ prior to stimulation. Imaging was performed on a Leica DMI8 microscope controlled by MetaMorph Imaging software. Cells were imaged at 5 frames $\mathrm{s}^{-1}$ for $1 \mathrm{~min}$ with a sCMOS camera (Photometrics Prime95B) and 20x magnification lens (Leica HCX PL FLUOTAR L 20x/0.40 NA CORR). $\mathrm{Ca}^{2+}{ }_{\text {-responsive mFAP-expressing cells }}$ were continually illuminated at $7.65 \mathrm{~mW} \mathrm{~cm}^{-2}$, and GCaMP6f-expressing cells were continually illuminated at $1.24 \mathrm{~mW} \mathrm{~cm}{ }^{-2}$, using a LumenCor Light Engine (Semrock filters: Excitation 474/27 nm; Emission 520/35 nm). Twenty seconds into the imaging experiment, a syringe pump (Harvard Apparatus Pump 11 Elite) was triggered by a TTL pulse. One-hundred microliters of Tyrode's solution spiked with the identical DFHBI concentration and percent DMSO, and $850 \mu \mathrm{M}$ acetylcholine (ACh) was added at a rate of $2 \mathrm{~mL} \mathrm{~min}^{-1}$ to the wells. In technical triplicate per sensor, the final conditions of each well were $850 \mu \mathrm{L}$ volume of $100 \mu \mathrm{M}$ ACh with constant DFHBI concentration throughout the addition of the ACh stimulation solution.

Data analysis: Regions of interest (ROIs) surrounding single cells from three technical replicates were hand-drawn in ImageJ software ${ }^{75}$. For each cellular ROI in each frame the average fluorescence was calculated in ImageJ. In order to perform background subtraction, cellular ROIs were then moved proximal to the original cell to an ROI where there was no fluorescence. Each cellular ROIs average fluorescence had the corresponding background fluorescence subtracted for each frame. Following background subtraction, fluorescence fold-change was calculated by Eq. (11) where $F$ is the background-subtracted average fluorescence per ROI per frame, and $F_{0}$ is the background-subtracted average baseline fluorescence as measured $1 \mathrm{~s}$ prior to ACh stimulation ${ }^{29}$. A maximal fluorescence response was determined as the maximum absolute value of the fluorescence fold-change after the stimulation frame (the peak $\left|\frac{\Delta F}{F_{0}}\right|$; Table 2), and a two-sided Wilcoxon rank sum test was used to compare values (Supplementary Fig. 18).

Cardiomyocyte imaging. hiPSC culture and cardiomyocyte differentiation: Undifferentiated IMR90 human induced pluripotent stem cells (hiPSCs) (WiCell) were maintained in mTeSR-1 (StemCell Technologies) on tissue culture plastic coated with Matrigel diluted 1:60 (Corning) at $37^{\circ} \mathrm{C}$ and $5 \% \mathrm{CO}_{2}$. Cardiomyocytes were differentiated using a monolayer-based directed differentiation protocol ${ }^{76}$. hiPSCs were dissociated into a single cell suspension using Versene (Life Technologies) and plated at a high density $\left(1.5-2.5 \cdot 10^{5} \mathrm{cells} \mathrm{cm}^{-2}\right)$ in mTeSR containing $10 \mu \mathrm{M}$ Y-27632 ROCK inhibitor onto Matrigel-coated plates. Twenty-four hours after plating, media was replaced with fresh mTeSR. Forty-eight hours after seeding, differentiation was induced by changing the media to $10-12 \mu \mathrm{M}$ CHIR 99021 (Stemgent) in RPMI 1640 supplemented with B27 minus insulin (RPMI-ins, ThermoFisher). After $24 \mathrm{~h}$ post-induction, the media was changed to fresh RPMIins. At 3 days post-induction, the media was changed to 3-5 $\mu \mathrm{M}$ IWP4 (Stemgent) in RPMI-ins. At 5 days post-induction, the media was changed to fresh RPMI-ins. At 7 days post-induction, the media was changed to RPMI medium containing B27 supplement with insulin (RPMI+ins), and media was changed every other day. Cardiomyocytes (CMs) were replated at day 14 post-induction in RPMI+ins at $1.5 \cdot 10^{5}$ cells $\mathrm{cm}^{-2}$. CMs were then purified via metabolic challenge by culturing in DMEM without glucose or sodium pyruvate, supplemented with $4 \mathrm{mM}$ sodium Llactate for 4 days ${ }^{77}$.

Recombinant adeno-associated virus ( $r A A V$ ) production and infection of hiPSC CMs: AAV-CAG-hChR2-H134R-tdTomato was a gift from Karel Svoboda (Addgene plasmid \# 28017; [http://n2t.net/addgene:28017]; RRID:

Addgene_28017), the vector backbone into which the EF1n_mFAP2b gene with sarcoplasmic reticulum (SR) targeting sequence was sub-cloned (Supplementary
Data 16). rAAV serotype 6 (rAAV6) were produced $^{78}$ with a titer for rAAV6-SREF1n_mFAP2b of $2.90 \cdot 10^{13}$ viral genomes $\mathrm{mL}^{-1}$. Purified hiPSC CMs in 6-well plates were infected with $3.00 \mu \mathrm{L}$ of the EF1n-mFAP2b rAAV6 construct at 30 days post-induction. Cardiomyocytes were incubated for 5 days before imaging. RPMI + ins media was replenished every 2 days without exchanging media.

Contraction and $\mathrm{SR} \mathrm{Ca}^{2+}$ transient imaging: Leica DMI8 microscope controlled by MetaMorph Imaging software was used to image contraction and $\mathrm{Ca}^{2+}$ transients of the SR in CMs. Prior to imaging experiments, CMs were rinsed with Tyrode's solution and the 6-well plate wells were filled with $2.0 \mathrm{~mL}$ of Tyrode's solution with $6.00 \mu \mathrm{M}$ DFHBI (Supplementary Fig. 19b) or $3.00 \mu \mathrm{M}$ DFHBI (Fig. 5f) from the stock solution. Cardiomyocytes were imaged at $\sim 16.7$ $\mathrm{Hz}\left(60 \mathrm{~ms}\right.$ frame $\left.^{-1}\right)$ for up to $24 \mathrm{~s}$ with a sCMOS camera (Photometrics Prime95B) and 20x magnification lens (Leica HCX PL FLUOTAR L 20x/0.40 NA CORR). The cells were continually illuminated at $5.72 \mathrm{~mW} \mathrm{~cm}^{-2}$ (Supplementary Fig. 19b) or $15.8 \mathrm{~mW} \mathrm{~cm}^{-2}$ (Fig. $5 \mathrm{f}$ and Supplementary Fig. 19c) with a LumenCor Light Engine (Semrock filters: Excitation $474 / 27 \mathrm{~nm}$; Emission 520/35 nm). For mapping contraction, three different ROI traces were hand-drawn, and displacement of cells were measured in the fluorescence channel. The normalized mean of the three ROI traces is plotted to visualize contraction of cardiomyocytes (Fig. $5 f$ and Supplementary Fig. 19b). In order to quantify tissue-level $\mathrm{Ca}^{2+}$ fluxes during cardiac contraction cycling, fluorescence fold-change over the whole field of view was calculated for each frame then temporally matched with contraction data. Fluorescence fold-change in each frame was calculated by Eq. (11) where $F$ is the fluorescence per frame, and $F_{0}$ is the fluorescence of the frame with the minimum fluorescence (Fig. $5 \mathrm{f}$ and Supplementary Fig. 19b). Temporal analysis was performed over 20 cardiac contraction cycles by averaging for each contraction the temporal difference between the peak whole field of view normalized fluorescence fold-change and the peak normalized average fluorescence from three ROI traces in the fluorescence channel (Fig. 5f).

Pharmacological inhibition of SERCA Ca ${ }^{2+}$ pumps: Cardiomyocytes in $2.0 \mathrm{~mL}$ of Tyrode's solution labeled with $3.00 \mu \mathrm{M}$ DFHBI were imaged at $2 \mathrm{~Hz}$ for $30 \mathrm{~min}$ with an sCMOS camera (Photometrics Prime95B) and 20x magnification lens (Leica HCX PL FLUOTAR L 20x/0.40 NA CORR). A stock solution of $20.0 \mathrm{mM}$ cyclopiazonic acid (CPA) (Tocris, 1235) in 100\% anhydrous DMSO was prepared Two milliliters of Tyrode's solution containing $3.00 \mu \mathrm{M}$ DFHBI and $40.0 \mu \mathrm{M} \mathrm{CPA}$ was administered at $\sim 10 \mathrm{~min}$ from initial image acquisition to inhibit SERCA pumps and disrupt $\mathrm{Ca}^{2+}$ recovery into the SR after contraction ${ }^{36}$. Fluorescence fold-change over the whole field of view in each frame was calculated by Eq. (11) where $F$ is the fluorescence per frame, and $F_{0}$ is the fluorescence at the first frame (Supplementary Fig. 19c).

Reporting summary. Further information on research design is available in the Nature Research Reporting Summary linked to this article.

\section{Data availability}

The atomic coordinates and experimental data of the EF1p2_mFAP2b-DFHBI-Ca ${ }^{2+}$ cocrystal structure have been deposited in the RCSB Protein Data Bank with the accession code $6 \mathrm{OHH}$. Time-lapse widefield epifluorescence microscopy movies of live COS-7 cells are available in Supplementary Movie 1 and Supplementary Movie 2. The normalized time-lapse widefield epifluorescence microscopy movie of live hiPSC-derived CMs expressing SR-targeted EF1n_mFAP2b labeled at $3.00 \mu \mathrm{M}$ DFHBI is available in Supplementary Movie 3. Amino acid sequences of mFAP variants are reported in Supplementary Data 1 and Supplementary Data 2. Other amino acid, DNA and oligonucleotide sequences used throughout this research are reported in Supplementary Data 3 through Supplementary Data 17. Plasmid DNA that support the findings of this study are available at Addgene [www.addgene.org/David_Baker]. Source data are provided with this paper and online [https://doi.org/10.5281/zenodo.3960743]. Other plasmid DNA and data that support the findings of this study are available from the corresponding author upon reasonable request. Computational models for mFAP2a, mFAP2b, mFAP10, the 59 extended loop decoys, 5 refined extended loop7 decoys, 8 circularly permuted mFAP2a or mFAP2b decoys, and 12 circularly permuted mFAP2a or mFAP2b decoys with designed linkers are available to download; the $\beta$-barrel loop fragment databases used to design the extended loop library, Supplementary Table 1, and Supplementary Table 2 are available to download [https://github.com/klimaj/mFAPs].

\section{Code availability}

The Rosetta macromolecular modeling suite [https://www.rosettacommons.org] is freely available to academic/non-commercial users and commercial licenses are available via the University of Washington Technology Transfer Office. The Rosetta design and refinement protocols for the extended loop library are available in Supplementary Note 2. The sequence design protocol to generate the combinatorial library of linkers to graft one EF-hand motif onto loop7 of mFAP2b is available in Supplementary Note 3. The PyRosetta design protocol to generate circularly permuted mFAP2a and mFAP2b is available in Supplementary Note 4 . The RosettaScripts script to design linkers onto cpmFAPs incorporates code in development and is available from the corresponding author upon request. Python scripts, RosettaScripts scripts, and parameterization files are available for download at [https://github.com/klimaj/mFAPs]. 
Received: 29 June 2020; Accepted: 10 August 2020;

Published online: 08 February 2021

\section{References}

1. Dou, J. et al. De novo design of a fluorescence-activating $\beta$-barrel. Nature 561, 485-491 (2018).

2. Strack, R. L. \& Jaffrey, S. R. New approaches for sensing metabolites and proteins in live cells using RNA. Curr. Opin. Chem. Biol. 17, 651-655 (2013).

3. Autour, A., Westhof, E. \& Ryckelynck, M. iSpinach: a fluorogenic RNA aptamer optimized for in vitro applications. Nucleic Acids Res. 44, 2491-2500 (2016).

4. Song, W., Strack, R. L., Svensen, N. \& Jaffrey, S. R. Plug-and-play fluorophores extend the spectral properties of spinach. J. Am. Chem. Soc. 136, 1198-1201 (2014).

5. Song, W. et al. Imaging RNA polymerase III transcription using a photostable RNA-fluorophore complex. Nat. Chem. Biol. 13, 1187-1194 (2017).

6. Paige, J. S., Wu, K. Y. \& Jaffrey, S. R. RNA mimics of green fluorescent protein Science 333, 642-646 (2011).

7. Tebo, A. G. et al. Circularly permuted fluorogenic proteins for the design of modular biosensors. ACS Chem. Biol. 13, 2392-2397 (2018).

8. Nagai, T., Sawano, A., Park, E. S. \& Miyawaki, A. Circularly permuted green fluorescent proteins engineered to sense Ca2+. Proc. Natl Acad. Sci. USA 98, 3197-3202 (2001).

9. Baird, G. S., Zacharias, D. A. \& Tsien, R. Y. Circular permutation and receptor insertion within green fluorescent proteins. Proc. Natl Acad. Sci. USA 96, 11241-11246 (1999).

10. Feng, S. et al. Improved split fluorescent proteins for endogenous protein labeling. Nat. Commun. 8, 370 (2017).

11. Cabantous, S. et al. A new protein-protein interaction sensor based on tripartite split-GFP association. Sci. Rep. 3, 2854 (2013).

12. Kerppola, T. K. Bimolecular fluorescence complementation (BiFC) analysis as a probe of protein interactions in living cells. Annu. Rev. Biophys. 37, 465-487 (2008).

13. Tebo, A. G. \& Gautier, A. A split fluorescent reporter with rapid and reversible complementation. Nat. Commun. 10, 2822 (2019).

14. Shu, X. Imaging dynamic cell signaling in vivo with new classes of fluorescent reporters. Curr. Opin. Chem. Biol. 54, 1-9 (2019).

15. Bozhanova, N. G. et al. Protein labeling for live cell fluorescence microscopy with a highly photostable renewable signal. Chem. Sci. 8, 7138-7142 (2017).

16. Plamont, M.-A. et al. Small fluorescence-activating and absorption-shifting tag for tunable protein imaging in vivo. Proc. Natl Acad. Sci. USA 113, 497-502 (2016).

17. Yan, Q. et al. Localization microscopy using noncovalent fluorogen activation by genetically encoded fluorogen-activating proteins. Chemphyschem $\mathbf{1 5}$, 687-695 (2014).

18. Tang, S. et al. Design and application of a class of sensors to monitor $\mathrm{Ca} 2+$ dynamics in high $\mathrm{Ca} 2+$ concentration cellular compartments. Proc. Natl Acad. Sci. USA 108, 16265-16270 (2011).

19. Tian, L. et al. Imaging neural activity in worms, flies and mice with improved GCaMP calcium indicators. Nat. Methods 6, 875-881 (2009).

20. Tantama, M., Hung, Y. P. \& Yellen, G. Imaging intracellular $\mathrm{pH}$ in live cells with a genetically encoded red fluorescent protein sensor. J. Am. Chem. Soc. 133, 10034-10037 (2011).

21. Mahon, M. J. pHluorin2: an enhanced, ratiometric, $\mathrm{pH}$-sensitive green florescent protein. Adv. Biosci. Biotechnol. 2, 132-137 (2011).

22. Fleishman, S. J. et al. RosettaScripts: a scripting language interface to the Rosetta macromolecular modeling suite. PLoS ONE 6, e20161 (2011).

23. Das, R. \& Baker, D. Macromolecular modeling with rosetta. Аnnu. Rev. Biochem. 77, 363-382 (2008).

24. Siedlecka, M., Goch, G., Ejchart, A., Sticht, H. \& Bierzynski, A. Alpha-helix nucleation by a calcium-binding peptide loop. Proc. Natl Acad. Sci. USA 96, 903-908 (1999).

25. Olsson, L. L. \& Sjölin, L. Structure of Escherichia coli fragment TR2C from calmodulin to 1.7 A resolution. Acta Crystallogr. D. Biol. Crystallogr. 57, 664-669 (2001).

26. Fallon, J. L. \& Quiocho, F. A. A closed compact structure of native $\mathrm{Ca}(2$ +)-calmodulin. Structure 11, 1303-1307 (2003).

27. Stefan, M. I., Edelstein, S. J. \& Le Novère, N. An allosteric model of calmodulin explains differential activation of PP2B and CaMKII. Proc. Natl Acad. Sci. USA 105, 10768-10773 (2008).

28. Busch, E., Hohenester, E., Timpl, R., Paulsson, M. \& Maurer, P. Calcium affinity, cooperativity, and domain interactions of extracellular EF-hands present in BM-40. J. Biol. Chem. 275, 25508-25515 (2000).
29. Chen, T.-W. et al. Ultrasensitive fluorescent proteins for imaging neuronal activity. Nature 499, 295-300 (2013).

30. Inoue, M. et al. Rational engineering of XCaMPs, a multicolor GECI suite for in vivo imaging of complex brain circuit dynamics. Cell 177, 1346-1360 (2019). e24.

31. Guo, J. \& Zhou, H.-X. Protein allostery and conformational dynamics. Chem. Rev. 116, 6503-6515 (2016)

32. Malmstrom, R. D., Kornev, A. P., Taylor, S. S. \& Amaro, R. E. Allostery through the computational microscope: cAMP activation of a canonical signalling domain. Nat. Commun. 6, 7588 (2015).

33. Hara-Kuge, S. et al. An improved inverse-type Ca2+ indicator can detect putative neuronal inhibition in Caenorhabditis elegans by increasing signal intensity upon Ca2+ decrease. PLoS ONE 13, e0194707 (2018).

34. Henderson, M. J. et al. A low affinity GCaMP3 variant (GCaMPer) for imaging the endoplasmic reticulum calcium store. PLoS ONE 10, e0139273 (2015).

35. Bovo, E., Martin, J. L., Tyryfter, J., de Tombe, P. P. \& Zima, A. V. R-CEPIAler as a new tool to directly measure sarcoplasmic reticulum [Ca] in ventricular myocytes. Am. J. Physiol. Heart Circ. Physiol. 311, H268-H275 (2016).

36. Seidler, N. W., Jona, I., Vegh, M. \& Martonosi, A. Cyclopiazonic acid is a specific inhibitor of the Ca2+-ATPase of sarcoplasmic reticulum. J. Biol. Chem. 264, 17816-17823 (1989).

37. Berger, S. et al. Computationally designed high specificity inhibitors delineate the roles of BCL2 family proteins in cancer. Elife 5, e20352 (2016)

38. Smith, E. M., Gautier, A. \& Puchner, E. M. Single-Molecule localization microscopy with the fluorescence-activating and absorption-shifting tag (FAST) system. ACS Chem. Biol. 14, 1115-1120 (2019).

39. Day, R. N. \& Davidson, M. W. The fluorescent protein palette: tools for cellular imaging. Chem. Soc. Rev. 38, 2887-2921 (2009).

40. Los, G. V. et al. HaloTag: a novel protein labeling technology for cell imaging and protein analysis. ACS Chem. Biol. 3, 373-382 (2008).

41. Abdelfattah, A. S. et al. Bright and photostable chemigenetic indicators for extended in vivo voltage imaging. Science 365, 699-704 (2019).

42. Liss, V., Barlag, B., Nietschke, M. \& Hensel, M. Self-labelling enzymes as universal tags for fluorescence microscopy, super-resolution microscopy and electron microscopy. Sci. Rep. 5, 17740 (2015)

43. Cooper, S. et al. Predicting protein structures with a multiplayer online game Nature 466, 756-760 (2010).

44. Huang, P.-S. et al. RosettaRemodel: a generalized framework for flexible backbone protein design. PLoS ONE 6, e24109 (2011).

45. Lin, Y.-R. et al. Control over overall shape and size in de novo designed proteins. Proc. Natl Acad. Sci. USA 112, E5478-E5485 (2015).

46. Rohl, C. A., Strauss, C. E. M., Misura, K. M. S. \& Baker, D. Protein Structure Prediction Using Rosetta. Methods Enzymol. 66-93. https://doi.org/10.1016/ s0076-6879(04)83004-0 (2004).

47. Alford, R. F. et al. The rosetta all-atom energy function for macromolecular modeling and design. J. Chem. Theory Comput. 13, 3031-3048 (2017).

48. Jacobs, T. M., Yumerefendi, H., Kuhlman, B. \& Leaver-Fay, A. SwiftLib: rapid degenerate-codon-library optimization through dynamic programming. Nucleic Acids Res. 43, e34 (2015).

49. Klima, J. C. et al. Bacterial expression and protein purification of minifluorescence-activating proteins. Protoc. Exch. https://doi.org/10.21203/rs.3. pex-1077/v1 (2021).

50. Li, Z. et al. Mutagenesis of mNeptune red-shifts emission spectrum to 681-685 nm. PLoS ONE 11, e0148749 (2016)

51. Godinho, L. F. \& Schrader, M. Determination of peroxisomal pH in living mammalian cells using pHRed. Methods Mol. Biol. 1595, 181-189 (2017).

52. Boassa, D. et al. Split-miniSOG for spatially detecting intracellular proteinprotein interactions by correlated light and electron microscopy. Cell Chem. Biol. 26, 1407-1416.e5 (2019).

53. Würth, C., Grabolle, M., Pauli, J., Spieles, M. \& Resch-Genger, U. Relative and absolute determination of fluorescence quantum yields of transparent samples. Nat. Protoc. 8, 1535-1550 (2013).

54. Olmsted, J. Calorimetric determinations of absolute fluorescence quantum yields. J. Phys. Chem. 83, 2581-2584 (1979).

55. Sjöback, R., Nygren, J. \& Kubista, M. Absorption and fluorescence properties of fluorescein. Spectrochimica Acta Part A: Mol. Biomolecular Spectrosc. 51, L7-L21 (1995).

56. Skinner, S. O., Sepúlveda, L. A., Xu, H. \& Golding, I. Measuring mRNA copy number in individual Escherichia coli cells using single-molecule fluorescent in situ hybridization. Nat. Protoc. 8, 1100-1113 (2013).

57. Otwinowski, Z. \& Minor, W. Processing of X-ray diffraction data collected in oscillation mode. Methods Enzymol. 307-326. https://doi.org/10.1016/s00766879(97)76066-x (1997)

58. Adams, P. D. et al. PHENIX: a comprehensive Python-based system for macromolecular structure solution. Acta Crystallogr. Sect. D. Biol. Crystallogr. 66, 213-221 (2010) 
59. McCoy, A. J. et al. Phasercrystallographic software. J. Appl. Crystallogr. 40, 658-674 (2007).

60. Emsley, P., Lohkamp, B., Scott, W. G. \& Cowtan, K. Features and development ofCoot. Acta Crystallogr. Sect. D. Biol. Crystallogr. 66, 486-501 (2010).

61. Afonine, P. V. et al. Towards automated crystallographic structure refinement withphenix.refine. Acta Crystallogr. Sect. D. Biol. Crystallogr. 68, 352-367 (2012).

62. Gumbart, J., Trabuco, L. G., Schreiner, E., Villa, E. \& Schulten, K. Regulation of the protein-conducting channel by a bound ribosome. Structure 17, 1453-1464 (2009).

63. Jorgensen, W. L., Chandrasekhar, J., Madura, J. D., Impey, R. W. \& Klein, M. L. Comparison of simple potential functions for simulating liquid water. $J$. Chem. Phys. 79, 926-935 (1983).

64. Maier, J. A. et al. ff14SB: improving the accuracy of protein side chain and backbone parameters from ff99SB. J. Chem. Theory Comput. 11, 3696-3713 (2015).

65. Case, D. A. et al. AMBER 14 (University of California, San Francisco, 2014).

66. Wang, J., Wolf, R. M., Caldwell, J. W., Kollman, P. A. \& Case, D. A. Development and testing of a general amber force field. J. Computational Chem. 25, 1157-1174 (2004).

67. Wang, J., Wang, W., Kollman, P. A. \& Case, D. A. Automatic atom type and bond type perception in molecular mechanical calculations. J. Mol. Graph. Model. 25, 247-260 (2006).

68. Frisch, M. J. et al. Gaussian 09, Revision C.01, (Gaussian, Inc., Wallingford CT, 2010).

69. Bradbrook, G. M. et al. X-Ray and molecular dynamics studies of concanavalin-A glucoside and mannoside complexes Relating structure to thermodynamics of binding. J. Chem. Soc., Faraday Trans. 94, 1603-1611 (1998).

70. Salomon-Ferrer, R., Götz, A. W., Poole, D., Le Grand, S. \& Walker, R. C. Routine microsecond molecular dynamics simulations with AMBER on GPUs. 2. Explicit solvent particle mesh ewald. J. Chem. Theory Comput. 9, 3878-3888 (2013).

71. Humphrey, W., Dalke, A. \& Schulten, K. VMD: Visual molecular dynamics. J. Mol. Graph. 14, 33-38 (1996).

72. Scherer, M. K. et al. PyEMMA 2: a software package for estimation, validation, and analysis of Markov Models. J. Chem. Theory Comput. 11, 5525-5542 (2015).

73. Roe, D. R. \& Cheatham, T. E. PTRAJ and CPPTRAJ: software for processing and analysis of molecular dynamics trajectory data. J. Chem. Theory Comput. 9, 3084-3095 (2013).

74. McGibbon, R. T. et al. MDTraj: a modern open library for the analysis of molecular dynamics trajectories. Biophysical J. 109, 1528-1532 (2015).

75. Schindelin, J. et al. Fiji: an open-source platform for biological-image analysis. Nat. Methods 9, 676-682 (2012)

76. Lian, X. et al. Directed cardiomyocyte differentiation from human pluripotent stem cells by modulating Wnt $/ \beta$-catenin signaling under fully defined conditions. Nat. Protoc. 8, 162-175 (2013).

77. Tohyama, S. et al. Distinct metabolic flow enables large-scale purification of mouse and human pluripotent stem cell-derived cardiomyocytes. Cell Stem Cell 12, 127-137 (2013).

78. Halbert, C. L., Allen, J. M. \& Chamberlain, J. S. AAV6 vector production and purification for muscle gene therapy. Methods Mol. Biol. 257-266. https://doi. org/10.1007/978-1-4939-7374-3_18 (2018).

\section{Acknowledgements}

J.C.K. would like to thank G. Reggiano for help extracting loops as .pdb files in designing the extended loop library, M. Glaz for assistance with laser scanning confocal fluorescence microscopy, J.B. Hurley for co-mentorship, and S. Berger, N. Ennist, A. Ford, H. Park, H. Shen, and other members of the Institute for Protein Design for helpful discussions. J.C.K. was supported by a National Science Foundation Graduate Research Fellowship (grant DGE-1256082). Part of this work was conducted at the Molecular Analysis Facility, a National Nanotechnology Coordinated Infrastructure site at the University of Washington, which is supported in part by the National Science
Foundation (grant NNCI-1542101), the University of Washington, the Molecular Engineering \& Sciences Institute, the Clean Energy Institute, and the National Institutes of Health. The Berkeley Center for Structural Biology is supported in part by the National Institutes of Health, National Institute of General Medical Sciences, and the Howard Hughes Medical Institute. The Advanced Light Source is a Department of Energy Office of Science User Facility under Contract No. DE-AC02-05CH11231. Any opinions, findings, and conclusions or recommendations expressed in this material are those of the authors and do not necessarily represent the official views of the funding agencies.

\section{Author contributions}

J.C.K., A.A.V., J.D., and D.B designed the research. J.C.K. performed: library assembly and screening; chromophore, $\mathrm{pH}$, and $\mathrm{Ca}^{2+}$ titrations; spectral, endpoint, and temporal fluorescence assays; densitometry; laser scanning confocal fluorescence microscopy of $E$. coli; photostability assays on fixed COS-7 cells; and CD. J.C.K. and J.S.Q. performed split mFAP titrations. L.A.D. performed X-ray crystallography. J.D.L. and M.R. cultured and transfected HEK293 cells. J.D.L. performed epifluorescence microscopy of HEK293 cell $\mathrm{Ca}^{2+}$ titrations. M.R. performed epifluorescence microscopy of HEK293 cell ACh stimulations. J.D.L. cultured, infected, and performed epifluorescence microscopy of hiPSCderived CMs. S.B. cultured and differentiated hiPSCs into CMs. L.A.G. cultured, transfected, and fixed COS-7 cells, and performed epifluorescence microscopy of live and fixed COS-7 cells. M.Y.L. measured quantum yields. E.P.B. performed molecular dynamics simulations. J.C.K., J.D., J.S.Q., C.M.C., and L.C. purified proteins. L.C. performed SECMALS. A.A.V. curated the $\beta$-barrel loop fragment databases and grafted them onto b11 and b32; J.D. designed mFAP2.0.1 and mFAP2.1; and J.C.K. designed all other proteins. D.B., B.L.S., A.B., J.C.V., R.E.A., and D.L.M. supervised research. All authors analyzed data. J.C.K. and D.B. wrote the manuscript with input from all authors.

\section{Competing interests}

The authors declare the following competing interests: J.C.K., A.A.V., J.D., and D.B. are coinventors on patent application PCT/US2019/025743, which incorporates the discoveries described herein. J.C.K. and D.B. have filed a provisional patent application $63 / 116,875$ on the methods and applications of split mFAPs and cpmFAPs. The remaining authors declare no competing interests.

\section{Additional information}

Supplementary information is available for this paper at https://doi.org/10.1038/s41467020-18911-w.

Correspondence and requests for materials should be addressed to D.B.

Peer review information Nature Communications and the authors thank the anonymous reviewer(s) for their contribution to the peer review of this work.

Reprints and permission information is available at http://www.nature.com/reprints

Publisher's note Springer Nature remains neutral with regard to jurisdictional claims in published maps and institutional affiliations.

(c) (i) Open Access This article is licensed under a Creative Commons Attribution 4.0 International License, which permits use, sharing, adaptation, distribution and reproduction in any medium or format, as long as you give appropriate credit to the original author(s) and the source, provide a link to the Creative Commons license, and indicate if changes were made. The images or other third party material in this article are included in the article's Creative Commons license, unless indicated otherwise in a credit line to the material. If material is not included in the article's Creative Commons license and your intended use is not permitted by statutory regulation or exceeds the permitted use, you will need to obtain permission directly from the copyright holder. To view a copy of this license, visit http://creativecommons.org/ licenses/by/4.0/

(C) The Author(s) 2021 